\title{
Molecular biology of oncogenic inflammatory processes. I. Non-oncogenic and oncogenic pathogens, intrinsic inflammatory reactions without pathogens, and microRNA/DNA interactions (Review)
}

\author{
JOSEPH G. SINKOVICS \\ Cancer Institute, St. Joseph's Hospital Affiliated with the H.L. Moffitt Comprehensive Cancer Center; \\ Department of Molecular Medicine, The University of South Florida College of Medicine, Tampa, FL, USA
}

Received September 26, 2011; Accepted October 20, 2011

DOI: $10.3892 /$ ijo.2011.1248

\begin{abstract}
In some inflammasomes tumor cells are generated. The internal environment of the inflammasome is conducive to the induction of malignant transformation. Epigenetic changes initiate this process. The subverted stromal connective tissue cells act to promote and sustain the process of malignant transformation. In its early stages, the premalignant cells depend on paracrine circuitries for the reception of growth factors. The ligands are derived from the connective tissue, and the receptors are expressed on the recipient premalignant cells. The initial events are not a direct attack on the proto-oncogenes, and thus it may be entirely reversible. Epigenetic processes of hypermethylation of the genes at the promoters of tumor suppressor genes (to silence them), and deacetylation of the histones aimed at the promoters of proto-oncogenes (to activate them) are on-going. A large number of short RNA sequences (interfering, micro-, short hairpin, non-coding RNAs) silence tumor suppressor genes, by neutralizing their mRNAs. In a serial sequence oncogenes undergo amplifications, point-mutations, translocations and fusions. In its earliest stage, the process is reversible by demethylation of the silenced suppressor gene promoters (to reactivate them), or re-acetylation of the histones of the
\end{abstract}

Correspondence to: Dr Joseph G. Sinkovics, St. Joseph's Hospital Cancer Institute, 3001 Martin Luther King Jr. Blvd, Tampa, FL 336076307, USA

E-mail: sinkovi.joseph@baycare.org

Key words: septic shock, inflammasomes, head and neck squamous cell carcinoma, mycoplasma oncogenesis, chlamydia lymphomagenesis, oncogenes, oncoproteins, epigenomics, microRNAs, glioblastoma, thyroid carcinoma, Barrett's esophagus, inflammatory breast cancer, pancreatic adenocarcinoma, colon cancer, prostate cancer, endometrial adenocarcinoma, sarcomas, melanoma, intrinsic inflammatory processes, Epstein-Barr virus-associated lymphomas, Kaposi's sarcoma, human papilloma virus-induced cancers, hepatocellular carcinoma, Helicobacter pylori, enterotoxigenic Bacteroides fragilis, urinary tract parasites, polyomaviruses, reactive oxygen nitrogen species, dsDNA damage and repair, ancient RNA/DNA complex, primordial DNA, oncogenic DNA oncogene promoters, thus de-activating them. The external administration of histone deacetylase inhibitors usually leads to the restoration of histone acetylation. In time, the uncorrected processes solidify into constitutive and irreversible gene mutations. Some of the pathogens inducing inflammations with consquential malignant transformation contain oncogenic gene sequences (papilloma viruses, Epstein-Barr virus, Kaposi's sarcoma-associated herpesvirus, hepatitis $\mathrm{B}$ and $\mathrm{C}$ viruses, Merkel cell polyoma virus, Helicobacter pylori, enterotoxigenic Bacteroides fragilis). These induced malignancies may be multifocal. Other pathogens are devoid of any known oncogenic genomic sequences (mycoplasma vav-carcinogenesis, chlamydia MALT-lymphoma genesis). In these cases the host's inflammatory reactions induce the malignant transformation in serial sequences of gene alterations initiated by hypoxia and reactive oxygen and nitrogen species generation. Carcinogenic intrinsic inflammatory processes endogenously initiated without a pathogen are recognized. Chronic inflammatory processes signal the RNA/DNA complex. In response, the DNA may revert into its ancient primordial 'immortal' format, which the clinics recognize as 'oncogenesis'. The DNA remains the ultimate master of bioengineering in order to sustain life. A discussion on the most versatile and resistant primordial RNA/ DNA complex and the pre-, proto-, and unicellular world in which they co-existed is included.

\section{Contents}

1. Pathogens without oncogenic genomic sequences activating cellular oncogenes

2. The inflammasome

3. Tumor cell colonies generated in the inflammasomes

4. Pathogens with potentially oncogenic genomic sequences

5. Mechanisms of inflammatory carcinogenesis

1. Pathogens without oncogenic genomic sequences activating cellular oncogenes

History. Credit goes to Jean-Nicolas Marjolin (1780-1850) for observing and reporting carcinogenesis in chronic fistulous 


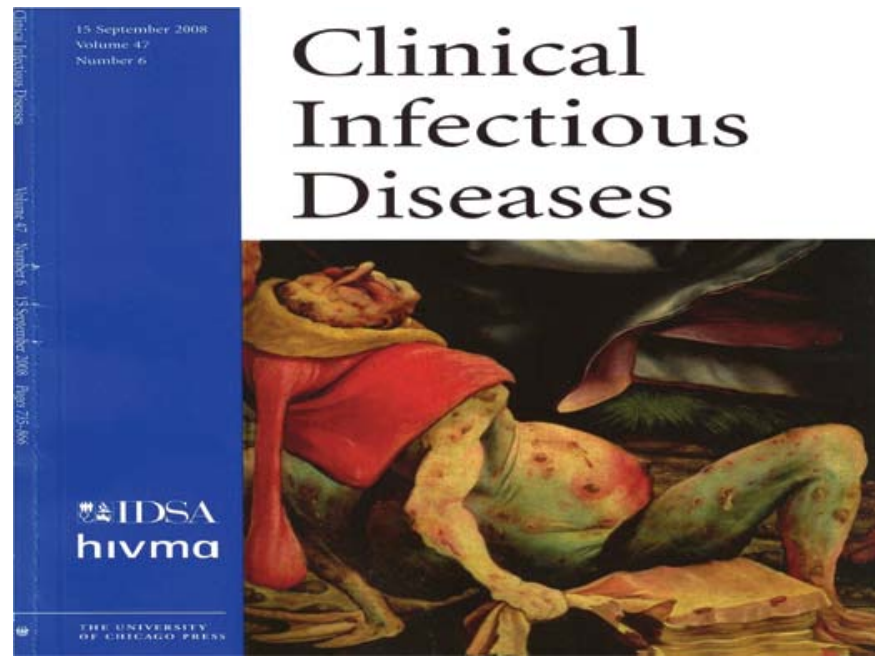

Figure 1. Altarpiece ‘The suffering man' by Matthias Grünewald, 1480-1528 Bread baked from wheat infected with Claviceps purpurea contained the toxin ergotamine. Consumption of this bread resulted in extensive severe vasculitis and bowel perforations. These patients died with hemorrhagic and septic shock The 'suffering man' is in the left lower corner of the painting. In the middle (not shown) St. Anthony is praying over the patient. The monks 'Brothers of St Anthony' stand behind awaiting for their turn to extend help and care to the dying man. Reprinted with permission.

tracts. He described the 'ulcéres verruques' in the hotbed of chronic infections (osteomyelitis with fistulous tracts): 'Ulcére de Marjolin sur ostéite chronique'. He became the Membre de l'Académie de Médicine Français, Paris, France. Contemporary histopathology confirms these lesions to be verrucous squamous cell carcinomas, which may originate in any chronic pustulous infected sites ('ulcéres suppurantes'), like in 'hidradenitis suppurativa' or in anal fissures ('papules péri-anales') and even in gingiva, tongue, and the oropharynx (vide infra).

Rudolf Virchow (1821-1902) described the inflammatory environment 'Phlogose und Thrombose im Gefässsystem' of malignant tumors ('die krankhaften Geschwülste'). Recently, Virchow was remembered in the Lancet for his early observation (1863) that chronic inflammatory processes accompany, and may even induce, cancers (1).

Septicemia. The monks 'Brothers of St. Anthony' recognized the high rate of mortality when they attended patients in the 1500 's, who suffered hemorrhagic and septic shock after consuming bread baked with Claviceps purpurea infected wheat. The alkaloid ergotamine of the fungus caused severe widespread vasculitis further complicated by septic shock, when guts perforated and peritonitis set in (Fig. 1) (2). High early mortality might have eliminated those patients who might have increased their susceptibility to late cancers due to suppressor gene silencing or oncogene activations during the acute illness.

Hemorrhagic and septic shock remained an acute complication of the highest mortality in the practice of medicine, especially when combination chemotherapy was introduced in medical oncology/hematology. This author attended patients with hemorrhagic and septic shock at the M.D. Anderson Hospital, University of Texas, Houston, TX, in the 1960's and 1970's (3-6). Those were the years when a leukemic patient with post-chemotherapy leukopenic fevers could die in pseudomonas septicemia with multiple vital organ failures within $24 \mathrm{~h}$.
A major recognition in the pathogenesis of hemorrhagicseptic shock is the role of the histone deacetylases. Histone deacetylation is balanced by the activities of histone acetyltransferases that transfer acetyl groups from acetyl coenzymes to lysines $(\mathrm{K})$ within the histone molecules. Histone deacetylases catalyze the removal of acetyl groups from the lysins. Histone deacetylases Class I, II, III, IV are preserved from the yeasts on upward in the evolutionary scale. Class I, II, and IV enzymes are zinc-dependent and occupy various intracellular localizations. Class III enzymes (sirtuins) are nicotinamide adenine dinucleotide-dependent. The genes of histone deacetylases are sensitive to inhibitors (HDACIs), and as such HDACIs exert neuro-, cardio-, and renal-protective effects in patients with hemorrhagic-septic shock. In the precancerous state, prominent is the activation of the PI3-kinase/Akt pathway (phosphatidyl inositol, akt transforming oncogene from thymic lymphoma of Jacob Furth's high leukemia Ak, later Rockefeller Institute AKR, mice), which is frequently activated as a cell-survival pathway in early events of carcinogenesis. Consequentially to PI3k/Akt activation, insulin-like growth factors (IGF), erythropoietin (EPO) and anti-apoptotic cytokines are activated. At the same time HDACIs inhibit Toll-like receptor TLR4, whose response to lipopolysaccharide (LPS) endotoxins is the activation of IL-1 $\beta$ and MyD88 (myeloid differentiation factor/scaffold) and IRAK (IL-1 receptor-associated kinase). Further suppressive effects of HDACIs are to the anti-apoptotic molecule Bcl-2, and the intranuclear transfer of $\beta$-catenin, which are frequently activated in early carcinogenesis. The pro-apoptotic tumor suppressor PTEN (phosphatase and tensin homolog deleted in chromosome 10: 10q23) may be inhibited by HDACIs, but this effect protects cardiomyocytes from apoptotic death. PTEN is an inhibitor of the PI3K/Akt pathway $(7,8)$.

Heat shock protein 70 (Hsp70) is induced by HDACIs, thus it exerts its cell-protective and anti-inflammatory effects. Member of the large family of chaperone Hsp, Hsp70, may protect proteins in cancer cells exposed to chemoradiotherapy. However, 2-phenylethyne-sulfonamide (PES) inhibits this tumor cell-protective effect (9). In squamous carcinoma cells, Hsp70 acting as a binding protein to Bcl-2-associated athanogene-1 (Bag-1) conveyed apoptosis-resistance. Hsp70 and Hsp90 protect metalloproteinase-2 in breast cancer cells and assist cancer cell migration and locomotion. Hyperthermia (or fever) mobilized Hsp70 to promote dendritic cell (DC) maturation, macrophage phagocytosis and pro-inflammatory cytokine (IL-8, IL-12) production (10-12).

Bacterial translocations to mesenteric lymph nodes, liver and spleen occur early in both hemorrhagic and septic shock. Epigenetic gene activations and gene silencing occur in the process. At lysine (K) 4 of activator histone 3, triple methylation (me3) occurs (H3K4me3) and results in silenced post-translational modifications. At lysine (K) 27 of suppressor histone 3, triple methylation (me3) occurs (H3K27me3) and results in up-regulated post-translational modifications (13). Endotoxin(LPS-) stimulated TLR4 activates myeloid differentiation factor 88, the scaffold protein (MyD88), IL-1 $\beta$, IL-6, IL-12 and TNF $\alpha$. Of the IL-1 receptor-associated kinases (vide supra) IRAK-1 translocates to the nucleus, where it activates STAT and IL-10. Variant IRAK-1 haplotype associates with nuclear factor $\kappa \mathrm{B}(\mathrm{NF} \kappa \mathrm{B}$, the reticuloendothelial virus' c-onc $\rightarrow \mathrm{v}-$ onc , Rel oncoprotein) overactivity; NFאB is liberated, transfers to the 
nuclei and through gene activations releases an excess of proinflammatory mediators. These events increase the fatal outcome of endotoxin shock (14). Endogenously released alarmins and damage-associated molecular patterns (DAMPs) act as ligands to TLR4. Such endogenous ligands of TLR4 are Hsp70 and some high-mobility group box 1 proteins (HMGB). Some of these reactions occur also early in oncogenesis (15). At the clinics, exogenously administered HDACIs antagonize practically all of these reactions (8). In the patients, it is the HMG protein B1 that performs DNA repair and chromatin modification after DNA damage. However, because of its role in mediating lethal systemic inflammation, HMGB1 is targeted for elimination. In contrast, HMGB1 in protecting against mutagenesis and acting for repairing DNA damage, it reduces or eliminates post-septic shock carcinogenesis, and for these reasons it should be saved (16). Indeed, the rate of post-septicemic oncogenesis does not appear to exceed that of the general population. However, the high mortality and short life span of patients in hemorrhagic/ septic shock might have eliminated those individuals who would have had experienced inflammatory carcinogenesis.

Variant IRAK-haplotypes regulate NFאB activation in sepsis and increased NFKB activity carries higher mortality (14), thus eliminating those patients who suffered oncogene activations. The saffron-derived carotenoid, crocetin, suppresses microRNA expression (in rat livers) for tumor necrosis factor $\alpha$ $(\mathrm{TNF} \alpha)$, interleukin-1 $\beta$ (IL-1 $\beta$ ) and inducible nitric oxide synthase (iNOS), thus alleviating the pathophysiology of the condition (17).

In septic shock, LPS-stimulated dendritic cells (DCs) excessively produce IL-6. MicroRNA 142-3p targets an untranslated region (UTR) of the IL-6 mRNA in DCs and thus reduces IL- 6 production. Consequentially mortality in septic shock increases. An oligonucleotide (locked nucleic acidmodified phosphorothioate oligonucleotide complementary to miRNA-142-3p) restores IL-6 production in DCs and reduces mortality (18). This report is difficult to comprehend, because it investigated only one pathway of the most complicated events active in septic shock, where IL-6 may act paradoxically both in pro- and anti-inflammatory contexts, and miRNA-142-3p acts also on lymphocytes and macrophages. Further, microRNAs $-9,-21,-146,-147$, and -155 are also active participants (18).

An interesting corollary is the function of microRNA142-3p in connection with the expression of fusion oncogene $m l l$ and its production of oncoprotein MLL (mixed lineage leukemia). The gene derives from the trithorax homologue of the drosophila; its locus in the human genome is at 11q23. It fuses quite promiscuously with various gene segment partners. When fused with AF4 proto-oncogene segment from $4 \mathrm{q} 31$ (asymmetric $f m r 2$ gene; $f m r 2=$ fragile mental retardation), it is associated with the induction of pro-B cell acute leukemias of newborn infants (19-21). The microRNA-142-3p targets the 3'UTR (untranslated) fragment of the mRNA from fused gene mll/af4 (17), thus it exerts anti-leukemia effects.

Epigenetic oncogenesis. Epigenetic events provide a link to oncogenesis and lead to the use of the new terminology 'epigenetic field for cancerization' and 'epigenetic switch linking inflammation to cancer' (22). One of the experimental supports for the use of the new terminology comes from the Harvard
Medical School. The activation of the $s r c$ proto-oncogene triggers $\mathrm{NF \kappa} \mathrm{B}$-mediated inflammatory response leading to the production of microRNA Lin-28 (lin = cell lineage abnormal in the nematode caenorhabditis and upward) and to the downregulation of microRNA Let-7 (let = lethal in caenorhabditis and upward). Let-7 being an inhibitor of IL- 6 production, its down-regulation leads to increased levels of IL-6. IL-6 activates the STAT3 pathway and further activates NFкB. STAT is an activator of microRNA-21 and miR-181b-1. These microRNAs inactivate the tumor suppressor genes PTEN and CYLD $(23,24)$ (PTEN $=$ phosphatase and tensin homolog on chromosome ten, 10q23; CYLD = cylindromatosis turban tumor suppressor gene on chromosome 16q12-q13).

In inflamed tissues, the short non-coding microRNA-155 inhibits the repair of dsDNA breaks, or allows mismatch repairs. These cells assume the 'mutator phenotype' and overexpress hypoxanthine phosphoribosyl-transferase to reflect to the excessive number of DNS breaks and mutations. Antibiotics (doxycycline), and LPS-induced inflammatory cytokines (TNF family members, IL-1 $\beta$, IL-6, IL-8) increase microRNA-155 expression in cancer cells. More dsDNA breaks follow. LPS-stimulated macrophage-conditioned medium imitates the effects of microRNA-155. The proliferation rate of adenocarcinoma cells is accelerated by microRNA-155. The cell cycle inhibitor Wee (small, in Scottish, by its discoverer Dr Paul Nurse working in Edinburgh), the wee gene product protein, the Wee kinase, is down-regulated by microRNA-155 (25). Thus, microRNA-155 removes the cyclin-dependent kinase (CDK) mitotic inhibitor, allowing uninhibited cell divisions to proceed. An anti-sense microRNA-155 iRNA (interfering RNA) neutralizes microRNA-155. The wee gene product protein WEE re-appears and mitoses come to a halt (26). The originally studied Schizosaccharomyces pombe cells with active Wee were nondividing and remained small (wee), whereas the dividing cells (no Wee) were large. The anti-mitotic tumor suppressor gene wee is often eliminated in human cancer cells (27). Inactivation of Wee by microRNA-155 is one of the mechanisms of inflammatory carcinogenesis (25-27).

Among other cancers, microRNA-155 is up-regulated in breast cancers. Three microRNAs (miR195, miRNA let-7a, miRNA-155) circulating in the blood of patients are practically diagnostic of breast cancer in $88 \%$ sensitivity and $91 \%$ specificity (28).

The Argonaute (AGO; the argonautes sailed for king Colchis' Golden Fleece guarded by dragons) family of proteins interacts with mi- and siRNAs and form miRNP complexes that regulate post-transcriptional gene expressions. The ancient AGO proteins in archaea and prokaryotes provide defense against intruding elements (phage genomes, retrotransposons). The modern AGO proteins are essential stabilizing core substances of the microRNA siRNA-induced gene silencing complexes (RISCs) (29,30).

The long intranuclear pri-miRs are processed by the intranuclear enzyme Drosha into 60-70 bp hairpin sequences (pre-miRs). Dicer loads the processed pre-miR sequences into RISCs. The cytoplasmic Dicer enzyme's (RNase endonuclease) substrate, the shRNAs, and/or the siRNAs are loaded into the AGO proteins. In human cells, Ago- 2 cleaves the ds siRNA to a ss RNA. The microRNA (mi-R) duplexes also form RISC complexes. Special mi-Rs are able to neutralize mRNAs 
traveling into the ribosomes to be translated into oncoproteins (31,32). Nanoparticle cyclodextrin polycations holding siRNAs complementary to the Ewing's sarcoma fusion oncoprotein Ews/ Fli1 (Ewing sarcoma breakpoint region I-flightless I drosophila homolog). In a mouse model of metastatic Ewing's sarcoma, this siRNA inhibited tumor growth (33). The CALAA-01 (cyclodextrin-containing polymer, synthetic nanoparticle formulated siRNA, human transferrin ligand aimed at the transferrin receptor) human clinical trial has been approved by the FDA $(34,35)$. The system prevents genomic intrusion, be it viral and/or oncogenic, thus it essentially prevents infection and inflammatory reactions to it, as it destroys the pathogen, be it viral, bacterial, or tumoral mRNAs, before its translation and multiplication.

Cancers originating in scars, chronic fistulous tracts and in lesions of periodontitis. It has been repeatedly observed throughout medical history that burn scars, scars left behind after zoster infections, or small pox vaccinations, chronic purulent fistulous tracts (anorectal, osteomyelytic, non-healing axillary hidradenitis) may convert in time commonly into 'verrucous' squamous cell-, rarely into adenocarcinoma (36-46). Unfortunately the genomics of these tumors were seldom if ever studied; only the documented cytological pathology reports are available as classical examples of inflammatory carcinogenesis: arising as verrucous squamous cell carcinomas in the chronic inflammasome.

There is no accepted proof confirming viral etiology (human papilloma virus, Merkel cell polyomavirus) for Marjolin ulcers or verrucous squamous cell carcinomas. In classical Merkel cell carcinomas, sequences of the viral genome are integrated in the host cell genome, and the viral $\mathrm{T}$ oncoprotein is mutated. In cutaneous squamous cell carcinomas of immunocompetent individuals, the polymerase chain reaction (PCR) was used for the detection of Merkel viral genomic sequences including those of the $\mathrm{T}$ antigen. Merkel polyomaviral infection was detectable in tumors of epidermodysplasia verruciformis, Bowen's disease, and some basal cell and sqamous cell carcinomas, but without $\mathrm{T}$ antigen mutations, or proven viral integration. However, in some squamous cell carcinomas, the Merkel polyomaviral genome was integrated, and the mutated $\mathrm{T}$ antigen was truncated. This finding was limited to only of $15 \%$ (26/177) of the squamous carcinomas tested (47-49).

At the Roswell Park Cancer Institute, Buffalo, NY, patients with chronic periodontitis were found to experience an increased incidence of squamous cell carcinomas of the tongue and the oral cavity $(50,51)$. Human papillomavirus carriers were not readily recognized (52), but bacteriological cultures point to a particular pathogen, Porphyromonas gingivalis. P. gingivalis may be dominant, but it is not alone: Chlamydia sp. and Prevotella sp. are also present in chronically inflamed gingival pockets (53,54a). As a fellow traveler, Epstein-Barr virus (EBV, vide infra) was frequently present in lesions of apical periodontitis (54b). $P$. gingivalis induces matrix metalloproteinase-9 (MMP) expression in human oral mucosal epidermal cells and dysregulates the expression of B7-H1 and B7-DC receptors in human gingival keratinocytes and squamous carcinoma cells (55-57). B7-H1, when expressed by DCs, induces the generation of antigen-specific $\mathrm{CD} 4^{+} \mathrm{Fox} 3^{+}$Treg cells, thus inducing tolerance toward selected antigens. Whereas, down- regulated $\mathrm{B} 7-\mathrm{H} 1$ receptorallows the generation of antigen-specific $\mathrm{CD} 4^{+} \mathrm{T}$ cell responses and antigen-specific IgG production. In contrast, B7-DC does not bind $\mathrm{T}$ lymphocyte-associated antigen CTLA-4; it induces interferon- $\gamma$ (IFN- $\gamma$ ), but not IL-4 and IL-10 secretion. Thus, B7-DC promotes the anti-tolerogenic Th1-type immunological environment. B7-H1 deficiency results in tolerogenic $\mathrm{CD}^{+}$and invariant iNKT cell generation (58-60). The advanced B7 systems are non-existent in teleost and bony fish that are in possession of the VDJ/RAG/RSS (variable, diversity, joining; recombination activating genes; recombination signal sequences) systems. The advanced B7 and ICOS (inducible $\mathrm{T}$ cell co-stimulator) systems were acquired later in the evolutionary scale $(61,62)$.

The epigenetics/genomics of squamous cell carcinoma generation. In head and neck and in particular in maxillary sinus squamous cell carcinomas the microRNAs miR-1 and miR-133a close to be knocked out. These are tumor suppressive microRNAs and their elimination is essential for successful carcinogenesis. In contrast, transgelin 2 and purine nucleoside phosphorylase levels rise in tumor tissues, when miR-1 and miR-133 are downregulated. Transgelin-deprived squamous cancer cells ceased to have mitoses and invasive activities $(63 \mathrm{ab}, 64)$. Further, therapeutic interventions influence the composition of tumor infiltrating lymphocytes. Naturally, the chemokine CCL22 invites the infiltration of anti-immune Treg cells. These are the $\mathrm{CD}^{+}{ }^{+} \mathrm{C} 25^{+} \mathrm{FoxP} 3{ }^{+}$tumor-supportive lymphocytes. Hyperthermia treatment encouraged an increased infiltration of both Treg and $\mathrm{CD}^{+} \mathrm{CD} 25^{-}$cells. Hyperthermia and irradiation induced only enhanced $\mathrm{CD} 4^{+} \mathrm{CD} 25^{-}$cell infiltration and resulted in decreased Treg cell infiltration without affecting the enhanced $\mathrm{CD} 4{ }^{+} \mathrm{CD} 25^{-}$cell infiltration (65).

The tumor suppressor protein, programmed cell death 4 (PDCD4), exerts also pro-inflammatory effects by up-regulating IL-6 and NFאB production, decreasing IL-10 production, and increasing the mortality of endotoxin (LPS) shock. In contrast, the PDCD4 antagonist, microRNA-21, is activated by the LPS/MyD88/NFאB cascade, or by IL-6 regulated activation of STAT3; then miR-21 exerts anti-inflammatory effects. As miR-21 down-regulates PDCD4, it raises IL-10 levels, thus establishing a PDCD4-deficient tolerogen internal environment. Other NFKB-induced inflammatory cytokines are TNF $\alpha$ and IL-1 $\beta$. Other consequences of PDCD4 loss is the activation of the PI3K/Akt 'cell survival pathway' commonly used by cancer cells. If an oligonucleotide knocked out miR-21, would the pro-inflammatory (consequentially pro-carcinogenic) effects of PDCD increase? The integrity of PDCD could be protected by preserving its mRNA by morpholino 21 (66).

Both IL-1 $\alpha \beta$ drive squamous carcinoma cells in the head and neck. Especially, cyclooxygenase-2 (COX-2) is activated. Oncoproteins Snail and Twist promote epithelial-tomesenchymal (EMT) transitions of the tumor cells. The Snail oncoprotein activates the colony stimulating factor-1 (CSF-1), which attracts macrophages; some of these macrophages convert into the M2/tumor-associated macrophage (TAM) lineage. The proliferation of IL-17/IL-6/STAT3-activated keratinocytes is driven by $\gamma \delta \mathrm{T}-17$ (IL-17 producing) lymphocytes. IL-17 induces IL-6 production in the macrophages. IL-6 activates 'cell survival cascade STAT3' in the keratinocytes. When IL-17 activates neutrophil leukocytes, endothelial cells and monocytes, it 
is pro-carcinogenic. When IL-17 promotes DCs, and activates NK cells and T lineage lymphocytes, it exerts antitumor effects. Tumor cells and their microenvironment with knock-out of STAT3 generate neutrophil leukocytes, and NK and T cells of anti-tumor efficiency. Within reactive DCs, the silencing of STAT3 breaks tumor antigen-specific T cell anergy. A siRNA oligonucleotide and the STAT3 antagonist stattic emerge as clinically effective inhibitors of the STAT3 pathway (67-70).

Patients with intraoral leukoplakia and dysplasia are at risk to advance to have squamous cell carcinoma. The gene CDK $\mathrm{N} 2 \mathrm{~A}$ encodes the p16 ${ }^{\mathrm{INK} 4 \mathrm{~A}}$ (inhibit CDK4) protein. Methylation of cytosine-post-guanine $\mathrm{CpG}$ islands in the genome of the gene silences it. Progression of oral epithelial dysplasia to squamous cell carcinoma begins with the silencing of the $16^{\mathrm{INK} 4 \mathrm{~A}}$ gene (71). In squamous cell carcinoma of the nasopharynx, promoter methylations in the $\mathrm{CpG}$ islands of the ubiquitin carboxyl terminal hydrolase 1 (UCHL1) gene opens up gates for carcinogenesis. The UCHL1 gene protects $\mathrm{p} 53$ and $\mathrm{p} 14^{\mathrm{ARF}}$ (alternative reading frame), which are doomed to ubiquitination, when the gene product proteins are fused (complexed) with the MDM2 protein (named after 'mouse double minus') human homolog gene product protein. The UCHL1 protein de-ubiquitinates the p53 and p14 ${ }^{\mathrm{ARF}}$ proteins and sends the MDM protein to ubiquitination. The $\mathrm{p} 14^{\mathrm{ARF}}$ protein protects the $\mathrm{p} 53$ protein, but only the UCHL1 gene product protein encoded from locus 4p11-14 is able to eliminate MDM2 (72). This is the tumor to whose etiology EBV's latent membrane proteins (LMP, vide infra) with or without the human papilloma virus 16's E6 and E7 oncogenes (vide infra) so decisively contributed.

Transforming growth factor $\beta$ receptors II and III (TGF $\beta$ R) are silenced in the epithelial layer and in the carcinomaassociated fibroblasts of oral squamous cell carcinomas. TGF $\beta$ I activates through IL-17 an inflammatory reaction in premalignant squamous cell lesions and thus inhibits the growth and metastases of squamous cell carcinomas in the tongue. This very same ligand down-regulated TGF $\beta$ RII and III, thus promoting tumor progression. The dual role of TGF $\beta$ consists of inhibitory effects on tumor growth, and/or tumor growth promotion through activation of myofibroblasts of the tumor microenvironment. The tumor-associated fibroblasts (myofibroblasts) produce keratinocyte growth factor (KGF) and matrix metalloproteinase (MMP) for paracrine receptors of the tumor cells. TGF $\beta$ ligands (decapentaplegic, from drosophila) activate the downstream mediator smad genes (signaling mothers against decapentaplegic, from the drosophila). Smad signaling operates within the MAPK, NFאB and sonic hedgehog ( $\mathrm{sHH}$ ) network. TGF $\beta$ ligands interact with miR-192 and miR377, but antagonize miR-29a; many more such interactions are awaiting recognition (73-76).

Oral cavity squamous carcinoma (OSCC) cells may use an overexpressed autocrine IL-6 $\rightarrow$ IL-6R circuitry for their own growth stimulation. The growth of human OSCC xenografts was inhibited by the anti-IL-6R humanized monoclonal antibody (mcab) tocizilumab. In the treated animals, the STAT3 pathway, and tumor-directed neoangiogenesis both were inhibited. Further, MMP-9 and IL-8 productions were also ablated. Thus, both autocrine and paracrine tumor growth factor circuitries were targeted by the mcab tocizilumab $(77,78)$. Other inhibitors of OSCC growth factors are anti-IL-8 siRNA; and aspirin and bortezomib for the proteasome/NFkB pathways (78). The strong efficiency of anti-inflammatory agents against OSCC proves that inflammatory processes initiate and sustain this tumor. In this case again, it is the epigenetics that initiated carcinogenesis. IL-6 induced global hypomethylation of the long interspersed nuclear element-1 (LINE-1), and executed silencing hypermethylations in the $\mathrm{CpG}$ islands of the promoters of tumor suppressor genes CHFR (checkpoint forkhead zink finger domain); GATA5 [for full sense and anti-sense GATA5M and GATA5U (79)]; and PAX6 (paired box genes) (80).

Rapidly enlarging OSCC tumor masses extend into avascular and hypoxic environments. These tumor cells can adapt to, and grow, in hypoxic environments. Both hypoxia inducible factors (HIF1 and HIF2) promote tumor cell divisions in hypoxic environments. Patients with tumors showing strong nuclear staining for HIFs experienced shortened overall and tumor-free survivals. Lentivirally encoded anti-HIF1/HIF2 shRNA short hairpin anti-sense molecules 1 and 2 knocked out both HIFs in OSCC xenografts and thus inhibited tumor growth (while shRNA-neg control exerted no such effects). The small molecular inhibitor NSC-134754 inhibited both HIFs in Hippel-Lindau gene-defective kidney carcinomas, thus it should be used for targeting against OSCC (81).

Amplification of $3 q$ is common in squamous cell carcinomas of mucosal origin rising from $37 \%$ to $92 \%$ in lung, uterine cervix, esophagus and head and neck $(\mathrm{H} \& \mathrm{~N})$ cancers. The locus of amplification in head and neck squamous cell carcinomas is the 3q26-27 region. Against 3\% or less in normal mucosa, it rises to $25 \%$ in dysplasia and carcinoma in situ up to over $56 \%$ in invasive carcinoma. In recurrent carcinomas the copy number of the gene amplifies up to $72-90 \%$ (82). The suspected gene is a member of the SOX gene family (SRY-related high mobility group transcription factor; SRY = sex-determining region in Y chromosome) (vide infra).

Small non-coding microRNAs (miR) and long non-coding RNAs are highly active in squamous cell carcinomas and in their niches. The down-regulated miR-489 is a wide-spectrum suppressor of the activated head and neck squamous carcinoma cell oncogene PTPN11 (protein tyrosine phosphatase nonreceptor). The cytoplasmic protein PTPN11 operates through two src homology domains (83). The miR-200c in squamous carcinoma stem cells switched off BMI1 signaling (Moloney leukemia virus insertion site; human polycomb gene product protein with tyrosine in its ring finger, instead of cysteine, that inactivates PTEN; degrades ubiquitin-proteasome) (84). When BMI1 was up-regulated, miR-200c levels were low. Overexpressed miR-200c down-regulates oncogenes Snail and ZEB1 (zink finger E box) and $\mathrm{N}$-cadherin, but up-regulates vimentin and E-cadherin (consequentially inhibiting the liberation and intranuclear transfer of $\beta$-catenin). The EMT and metastatic movements of squamous carcinoma cells with up-regulated miR-200c were inhibited (85).ZEB1 is the master regulator of EMT (the other EMT-inducer gene product proteins are Snail, Twist, Slug); microRNAs miR-200abc down-regulate ZEB1 (86).

At the 3p14 locus, the FHIT gene (fragile histidine triad) is deleted, while the epidermal growth factor receptor gene (EGFR) is overexpressed in $50 \%$ of squamous cell carcinomas. EGFR co-amplifies with CCND1 (cyclin D1) (87). Oncogenes overexpressed in smokers' squamous cell lung cancers are the SOX2 (vide supra et infra) and BRF1,2 (transcription factor 
IIIB-related factor, RNA polymerase III transcription initiator butyrate response factors) at 8p12 (88). The adenylate- and uridylate-rich elements (AU/ARE) are present in the untranslated region (3'UTR) of the mRNAs. The ARE-binding tristetraprolin proteins (among the BRF1,2) regulate the expression of canceror inflammation-associated proteins by attaching to 3'UTRs of mRNAs for their rapid degradation ('mRNA decay') (89). The mutated BRF genes fail to perform.

In the skin undifferentiated keratinocytes rise from the basal layer up to the differentiated suprabasal layer, where they die to form the stratified barrier lining. The activator protein (AP) transcription factors are part of the complex regulatory process. Inactivation of the AP factors results in hyperproliferation of undifferentiated keratinocytes, which form hyperkeratosis but not tumors (90). The gene product protein E2F (cyclin E inducer factor) brings about the cyclin/CDK2 complex. The E2F1mediated activation of p19(INK4D) results in the inhibition of CDK4 and CDK6, bringing the cell cycle to a halt in G1. Thus E2F establishes the INK4/pRB/E2F and p19/(ARF)p53 (alternative reading frame) tumor suppressor pathway (91-93).

Inflammation-induced (and other) cancers frequently activate the insulin-like growth factor receptor (IGF-R) pathway, which includes the up-regulated signaling of the Akt (vide supra) cell survival pathway (94). Some of the undifferentiated $\mathrm{H} \& \mathrm{~N}$ carcinomas with up-regulated IGF-R were $\mathrm{EBV}^{+}$and/ or $\mathrm{HPV}^{+}$(vide infra) (96). Two microRNAs (miR-7, miR-375) target the IGF-R1 mRNA, and thus inhibit the growth of squamous cell carcinomas $(94,96)$. However, promoter gene methylations of miR-375 in tumor-bearing patients annuls this effect (96). Monoclonal antibodies directed at EGF-R or IGF-R (cetuximab, A12, MK-0646, dalotuzumab) exert growth inhibitory effect on squamous carcinoma cell in vivo, also in clinical trials $(97,98)$.

Xeroderma pigmentosum $(X P)$. First described by Hebra and Kaposi and again by Kaposi as such, illustrated, in the Wiener Medizinische Jahrbuch, pp619-632, 1882. UV light-induced subcutaneous inflammation and dsDNA breaks remain unrepaired in patients with germ-line deficiencies of DNA repair systems (the defective helicases). The ERCC/TFIIH multiprotein multienzyme system carries out the repair of dsDNA breaks (excision repair cross-complementing; transcription factor for RNA polymerase II and XP helicases). The TFIIH opens the double helix; its enzymes are ATP-dependent helicases, 3-subunit CDK-activating kinases and the cdk. Polymorphisms of the ERCC/XPD system result in the failure of the nucleotide excision repair genes, referred to as excision repair cross-complementing repair deficiency, complementation 2 XPD (ERCC2) (99-102).

UV light-induced inflammatory changes and polymorphism in the nuclear excision repair gene complex increases the susceptibility to basal cell carcinoma (103). In BCC, overexpression of the $\mathrm{sHH}$ pathways dominates: HIP (HH interacting protein) overexpression and the deletion of the antagonist dickkopf proteins (from 'fat-head' drosophila) dominate. The WNT (wingless, drosophila), E-cadherin/ $\beta$-catenin and the mTOR (mammalian target of rapamycin) pathways are activated $(104,105)$. The $\mathrm{sHH}$ system was named after the 17-year-old hedgehog escaping from the planet Mobius for continuation of his mischievousness on the planet Earth (Sega video).

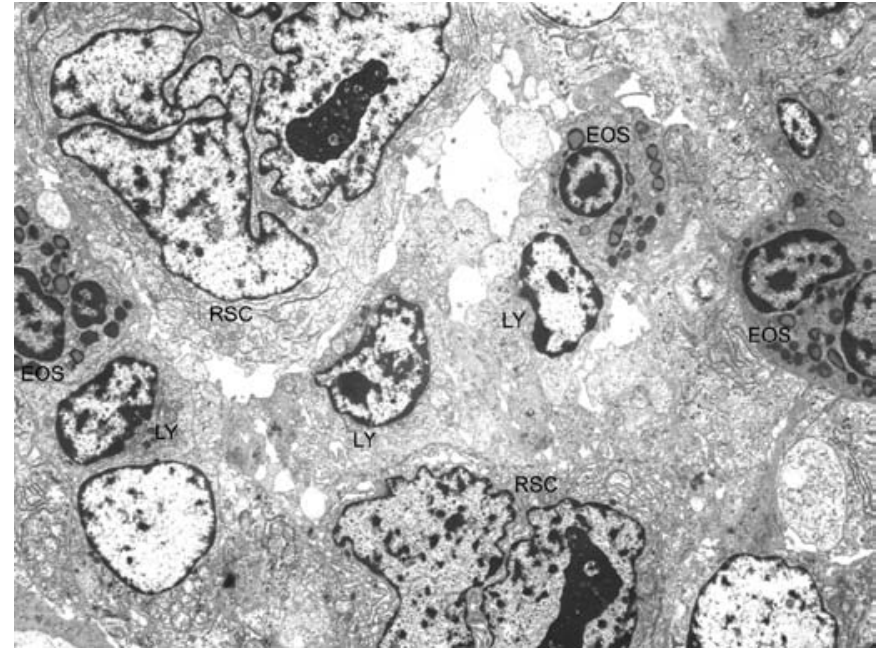

Figure 2. Eosinophil granulocytes in Hodgkin's disease granuloma. The ReedSternberg cells are intact, the lymphocytes are inert (refraining from attack). Original magnification, x12,500. Transmission Philips electron microscopy from the material of the Veterans' Medical Center, Department of Pathology (Head, Professor Ferenc Györkey), Houston, TX.

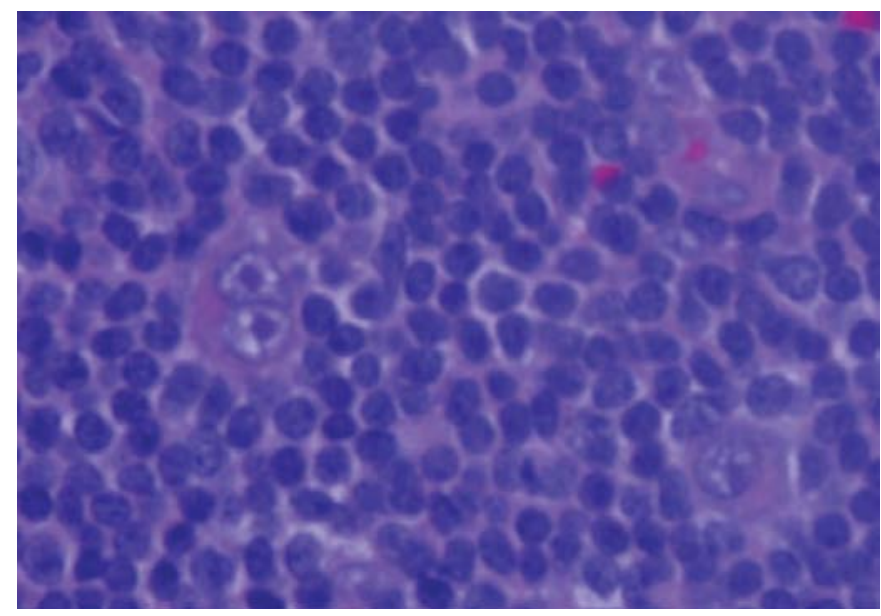

Figure 3. Intact Reed-Sternberg cells in lymphocyte-rich Hodgkin's disease granuloma are surrounded by $\mathrm{CD} 25^{+}$Treg cells for protection against immune $\mathrm{T}$ and NK cells. Original magnification, $\mathrm{x} 1,000$. From the patient material of St. Joseph's Hospital, Tampa, FL.

\section{The inflammasome}

An environment conducive to DNA transformation. These ancient formations are the battlefields between invasive pathogens and the host. Microbial molecules (flagellins; LPSs), and the pathogen-associated molecular patterns (PAMPs), are recognized by the Toll-like receptors (TLRs). Granulocytes and monocytes (macrophages) were the defensive cells in the era of innate immunity; so were dendritic cells and NK cells. Eosinophilic granulocytes were selected out as potential defensive cells against inflammatory carcinogenesis (106). If so, they may be failing to defend the host against Reed-Sternberg cells in Hodgkin's disease granulomas (Fig. 2). The Treg cells surround Reed-Sternberg cells protecting them against immune T cells (Fig. 3). Apparently eosinophil granulocytes fail to break through these barriers.

Caspase- 1 activates the inflammatory cytokines IL-1 $\beta$ and IL-18 by cleavage into their active form. Alarmin, the high 
mobility group B protein is released. By the process of pyroptosis, pathogen-carrier host cells (macrophages) are killed. The pathogens mobilize virulence islands and alter their surface antigenic networks. Poxviruses release soluble IL-1 $\beta$ receptors and IL-18 inhibitors. Multimers are formed by nucleotidebinding leucin-rich repeats-containing proteins (NLRP). The Nlrp3 component invites myeloid-derived suppressor cells (107). Apoptosis-associated speck-like proteins (ASC), caspase recruit domains (CARDs), and transmembrane segments (TMS), also known as ASC and PYCARD (PYD = pyrin domain) are activated. The intracellular nucleotide-binding oligomerization domains (NOD) recognize pathogens that penetrated the cell membrane. IFN $\gamma$-producer immune $\mathrm{CD}^{+} \mathrm{T}$ cells are in combat with Treg cells and myeloid-derived suppressor cells (MDSC). Chemokines, on the side of Treg cells, direct them against immune T cells. NLRPs generate MDSCs (reviewed in ref. 108). Outstanding reviews analyze in details the operation of inflammasomes against all classes of pathogens (viral, microbial, fungal, protozoal) (109-113), and all the counteractions of the pathogens, but make no mention just how oncosuppressor genes become silenced and oncogenes become activated within the inflammasomes. However, the figures (cartoons) allow an insight. In the A. Skeldon and M. Saleh paper (111), the appearance of the anti-apoptotic $\mathrm{Bcl}-2$ and $\mathrm{Bcl}_{\mathrm{XL}}$ proteins could protect incipient tumor cells from apoptotic death. In the P.K. Anand, R.K. Subbarao Malireddi and T.-D. Kanneganti paper (113), $\mathrm{NF \kappa B}$ appears as it translocates from cytoplasm to nucleus. This is a common event in oncogenesis, whereby tumor growth factor cytokine genes are activated. There is very little if any mention of epigenomics; reactive oxygen species and dsDNA breaks, etc. The most complex and not uniform composition of the inflammasome may be carcinogenic, or lymphomagenic. The precise mechanisms of intra-inflammasome oncogenesis are not well understood. An attempt at describing these events in the context of inflammatory oncogenesis will be made later in this article.

Mycoplasma oncogenesis. In the mid 1960's, the National Cancer Institute alerted R. Lee Clark, the president of M.D. Anderson Hospital, that reports from European countries (England, France, Germany and Moscow, Russia, then the Soviet Union) claim that strains of Mycoplasmataceae are being frequently isolated from the blood and bone marrow of leukemic patients and that these microorganisms may be etiologic (leukemogenic) agents. The British reports were selected out as the most prominent ones $(114,115)$. The reports from Ann Arbor, MI (116) indicated pathogenicity of certain human mycoplasma isolates in mice (toxic deaths, inflammatory conditions, 'leukemoid reactions'). The NCI was to release a substantial special grant for the study of these microorganisms in the context of etiological importance in acute and chronic leukemias. This author was elected to be the principal investigator of the project, based on his earlier publications describing 'pleuropneumonialike growth of bacteria' $(117,118)$, which elicited a letter and a citation from Joshua Lederberg (119), and an expression of interest in the project by Albert Sabin (120). The pleuropneumonia microorganisms were either true mycoplasmas, or some bacteria growing like pleuropneumonia microorganisms.

A new laboratory was immediately equipped for the growth of mycoplasmas (or pleuropneumonia-like organisms, PPLO) from specimens of human origin (saliva, urine, blood, bone marrow) collected and provided for the laboratory by the principal investigator, J.G.S. Florence Pipes was recruited from New Orleans to be in charge of the cultures of these microorganisms. Leon Dmochowski, head of the Department of Virology, appointed his technician Bernadette Borchers to prepare the specimens for electron microscopy studies. The principal investigator (J.G.S.) inoculated several hundreds young suckling white Swiss mice intraperitoneally and/or intravenously through a prominent lateral facial vein for observation. The observation consisted of very frequent inspection for 'general health', palpation of lymph nodes and spleens, periodical examination of blood counts and blood smears of the inoculated mice. Mice succumbed early after inoculations showed 'infectious diseases pathology' without any malignant features. However, practically all other mice remained healthy by one full year. Even after sacrificing many of them for internal examination by histopathology, not one case of leukemia or solid tumor (sarcoma, carcinoma) was observed. The cultures of human sources yielded various strains of mycoplasmas in less than $20 \%$ of the patients; some mycoplasma-like microorganisms grew out as bacteria $(E$. coli) from patients who were receiving an antibiotic when their blood samples were collected. However, the electron microscopic studies showed spectacular pictures of mycoplasma-like microorganisms (sometimes in cases when the cultures were 'no growth'). The principal investigator's (J.G.S.) report at first year's end was in the 'negative'. Neither the Koch postulates could be verified, nor the oncogenic pathogenicity of the cultured mycoplasma microorganisms (like the most frequently isolated $M$. salivarius) could be proven. In the second year of the project, Dmochowski assumed the position of the principal investigator, as he and Clark suggested to the NCI the need for 'further studies', which remained well financed. In the 4th year's final report of Dmochowski, the conclusion remained in the negative: no leukemo- or oncogenicity of the mycoplasma isolates could be documented by the technology applied (121).

However some 30 years later, a mycoplasma strain, the $M$. fermentans incognitus, emerged as a suspect co-etiologic factor in patients with the acquired immunodeficiency syndrome (AIDS) $(122,123)$. Extensive laboratory studies on this strain of mycoplasma eventually failed to show any unique faculties that would distinguish it from the common other strains of M. fermentans (124). Even though, AIDS-related B-lineage highly malignant lymphomas show gene deletions, among them that of the tumor suppressor gene WWOX (double tryptophan WW domain; osteosarcoma oxidoreductase, vide infra) (125), mycoplasma microorganisms were not reported (so far) as being visualized in the tissue sections. Now, over 35 years after the negative M.D. Anderson Hospital studies, reports appear of mycoplasma-infected human tumors (adenocarcinomas) in the $40-50 \%$ range $(126,127)$. It is recognized that the presence of a pathogen does not mean causation without fulfilling the Koch's postulates.

M.fermentans and M.penetrans cultured from patients with AIDS were inoculated into cultured mouse embryo cells at the Armed Forces Institute of Pathology, Bethesda, MD. Progressive multistage malignant transformations (MT) were observed. Early stages of MT were reversed upon mycoplasma eradication with antibiotics (ciprofloxacin). Advanced stages of MT became irreversible (constitutive). Chromosome breaks and deletions were observed during MT, but insertion of mycoplasma genomic 
sequences into tumor cell nuclei could not be documented. Tumor cells grew into tumors in nude mice, first very slowly, then in accelerated rate, upon serial passages. Tumor cells showed increased expression of G-coupled proteins, overexpressed chemokine receptors and expressed Ras and Vav oncoproteins. In advanced mycoplasma-induced tumor cells, the $\mathrm{Rb}$ and $\mathrm{p} 53$ tumor suppressor genes are down-regulated (128-130). Even though the initial oncogenesis occurred in vitro in embryonic cell cultures, the authors state: 'Our previous studies have revealed that chronic and persistent infection with these seemingly lowvirulence mycoplasmas could gradually but significantly affect many important biological characteristics of mammalian cells and even lead to malignant transformation' (130). However, were innate or adaptive immune reactions generated in the embryonic cell cultures?

The vav family proto-oncogenes (vav1, 2, 3) show src homology, encode nerve cell dendrites, axons and ephrins in Schwann cells in embryonic life, activate $\mathrm{NF \kappa B}$ in B lineage lymphocytes, reorganize actin cytoskeletons, act as guanine exchange factors, thus activating Rho GTPases, and accelerate cell mitoses. Vav stands for the sixth letter of the Hebrew alphabet and means 'link' or 'connection'. Vav is emerging as a newly recognized powerful oncoprotein. In an article of spectacular illustrations, Vav is described as guanine nucleotide exchange factor for the Rho (Ras homologous) family GTPases (its $d b l$ homology DH domain, the diffuse B large cell lymphoma oncogene), displaying seven other (eight) homologies; among them calponin and pleckstrin homology, zink finger and Src homologies. A truncated Vav is the cell-transforming oncoprotein. The vav proto-oncogene was exclusively expressed in hematopoietic cells (and was first found to be silent in epithelial, mesenchymal and neuroectodermal cells). Active vav proto-oncogene was detected in some autoimmune diseases (131-136). The human vav protooncogene maps to chromosome region 19p12-12.2 linked to the insulin receptor locus (137).

When human malignancies display the VAV oncoprotein: is there a good reason to suspect an etiological connection with prior or persistent chronic mycoplasma infection (with any strain of mycoplasma, or with only the M. fermentans, $M$. penetrans strains)? Would serological studies clarify this issue in non-smokers with Vav oncoprotein-positive lung cancers? Or, in patients with Vav oncoprotein-positive prostate cancer and positive serology for genitourinary mycoplasmas (vide infra)? Vav1 is expressed in some human lung cancers and Vav3 in some prostate cancers (vide infra) $(138,139)$.

Chlamydia lymphoma-genesis. These pathogens notoriously induce chronic infections. In old textbooks these agents were mistaken for large viruses (trachoma virus; psittacosis virus, see in Sinkovics' Die Grundlagen der Virusforschung), but the characteristics of their multiplication was recognized to be that of 'intracellular bacteria, or mycoplasma' (117). Chlamydia sp. notoriously infect the uterine cervix and the prostate $(140,141)$. In the uterine cervix, it may co-exist with human papilloma viruses (140).

C. psittaci is a well-established etiological agent of human marginal zone and mucosa-associated lymphatic tissue (MALT) lymphomas. The matter was debated 'against versus for' in Blood with evidence supporting more 'for', than 'against' (142-144).
Periocular adnexal lymphomas are the most common tumors $(145,146)$, but in one case chlamydia-carrier marginal zone lymphoma originated in the choroid plexus of the brain without concomitant periocular involvement. Monocytes/macrophages in the lesions carried the pathogen (147). Chlamydia antigen-driven $\mathrm{B}$ cells undergo polyclonal expansion. In this stage oncogenes are not yet activated. The process is reversible by eradication of chlamydia with antibiotics (148). By MALT-like translocations favoring $\mathrm{NF} \kappa \mathrm{B}$ activation (vide infra at Helicobacter pylori), monoclonally expanding B cell population(s) emerge (149). The chlamydia heat shock protein (HSP60) released intracellularly exerts anti-apoptotic effect, thus the expansion of the B cell clones continues (150).

$B$ cell clones with the translocation $t(11 ; 18)$ were free of chlamydia, but the $\mathrm{NF \kappa B}$ activation remained constitutive. The involved cell clones suffered mutations or deletions of the A20/ TNFAIP3 gene (tumor necrosis factor $\alpha$-induced protein; A20 ubiquitin-modifying anti-NFאB enzyme at $6 \mathrm{q}$, deleted also in AIDS- and EBV-related lymphoma). The gene product proteins of these genes act as suppressors of NFKB. Further, the promoter of the p16/INK4 $\alpha$ gene (inhibitor cyclin-dependent kinase 4) was silenced by hypermethylation. The cartoon in the cited article (151) shows genetic predisposition, chronic antigenic stimulation of B lineage cells (once rendered constitutive, the B cell proliferation continues after the disappearance of the antigen of chlamydia derivation), two genetic translocations: $t(11 ; 18)$ and $\mathrm{t}(14 ; 18)$, p16 alterations, DC-induced helper T cell generation,

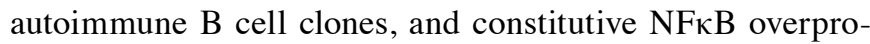
duction (151). With co-authors, this author presented human patients with malignant lymphomas from the clinical material of M.D. Anderson Hospital, in which cases chronic antigenic stimulation (not that of chlamydia) initiated the proliferation of lymphoid cell clones. Some of the antigens were auto-antigens and the lymphoid cell clones acted as foreign grafts attacking the host (causing a graft-versus-host-like autoimmune disease underlying the malignant lymphoma) (152). If an unidentified chlamydia antigen mimics host cell antigens, even after the disappearance of the pathogen, the immune reactions against that antigen may continue both as an autoimmune disease and as a malignant lymphoma. Thus, periorbital B cell lymphomas occurring in Africa (Kenya), but without chlamydia microorganisms being documented in the lesions (153), they still might have been initiated by $C$. psittaci. It appears as if chlamydia initiated in vivo an inflammatory process confined to lymph nodes, not carcinogenic, but lymphomagenic.

\section{Tumor cell colonies generated in the inflammasomes}

Glioblastoma multiforme (GMF). This highly malignant and radio-chemotherapy-resistant tumor expropriated the FasL $\rightarrow$ FasR pathway for driving cell cycle progression (154). The Fas receptor- (FasR) and granulocyte colony stimulating factor- (GCSF) encoding gene sequences could be artificially recombined in vitro. Previously, we presumed that such mismatched fusions of broken chromosomes 1 and 10 occur in melanoma in vivo (vide infra). These tumor cells undergo mitoses upon capturing FasL. The microglia and astrocytes interact with tumor cells either in an inhibitory or in a stimulatory fashion. It is usually unknown what agents activate these reactions, especially at the earliest tumor-induction phase. In astrocytes 
the activated TLR4 induces the IL $\beta-1 / \mathrm{MyD} 88 / \mathrm{NF} \kappa \mathrm{B}$ signaling pathway resulting in the mobilization of the 'cell survival' MAPK and Jak1STAT1 (janus kinase) chain reaction (155). Rapidly growing tumors (glioblastoma) advance into hypoxic territories. There, in hypoxic tumor cells, hypoxia-inducible transcription factor HIF1 $\alpha$ translocates into the nucleus to bind HIF1 $\beta$. The HIF1 $\alpha \beta$ dimer induces neoangiogenesis outside the cell and apoptosis-resistance within the cell. Further, hypoxia responsive elements (HRE) on inflammatory gene promoters (COX, NOS, PTX, CXCR-4, SDF-1 $\alpha$ ) capture HIF1 $\alpha$ (cyclooxygenase, nitric oxygen species, acute phase protein pentraxin, chemokine, stromal-derived factor). In response, tumor cells proliferate ( $\uparrow \mathrm{Ki67}$ ) and migrate; and resting stem cells undergo activation and enter the cell cycle. These stem cells are vulnerable to malignant transformation instead of differentiation (156).

Adenosine $\rightarrow$ adenosine receptor $\mathrm{A}(1) \mathrm{R}$ interactions suppress glioblastoma growth and cellular invasion. $\mathrm{A} \mathrm{AR}^{+}$microglia cells inhibited the growth and invasion of glioblastoma cells. The nucleoside adenosine derives from the nucleotide ATP. In contrast, A2AR stimulation by its ligand results in the expression of cyclooxygenase-2, prostaglandin and nerve growth factor (NGF). Activated A1AR suppresses MMP production in the tumor (157). These tumors contain microglia cells and macrophages and originate from stem cells (158). In addition to up to $30 \%$ microglia in the tumor mass, and some subverted not transformed astrocytes also provide tumor growth factors (159). One of the growth factors is TGF $\beta$ acting through its receptor TGF $\beta I I R$. Small hairpin complementary shRNA in a plasmid down-regulated expression of the receptor, and thus reduced tumor cell growth and invasiveness in nude mice (160).

J.C. Horvath and this author submitted a hospital surveillance committee-approved protocol for the immunotherapy of GMF with viral oncolysate-stimulated immune T lymphocytes and lymphokine-activated NK cells (LAK cells), which was not funded, for those clinical investigators, who possibly could obtain the financial support (161). This article provided a review on the interactions of astrocytes and microglia with the tumor cells (based on work being carried out up to 2006 at M.D. Anderson Hospital). It concluded that after the eradication of the tumor bulk by surgery and radiotherapy, it will be immunotherapy that will prevent the relapse with resistant tumor cells. At M.D. Anderson and elsewhere, a vaccine of bone marrow-derived dendritic cells pulsed with tumor homogenates was recommended $(162,163)$. A genetically engineered oncolytic adenovirus was another choice (reviewed in ref. 164). The splice variant of the epidermal growth factor receptor EGFRvIII vaccine was already in its earliest clinical trials. The article (161) recommended a viral oncolysate vaccine subcutaneously, and adoptive immune T cell/NK cell therapy to be administered through an Ommaya reservoir into the tumor bed. In support, it was known that the brain readily accepted extravasated immune $\mathrm{T}$ and NK cells (165-167). NK cells attack glioblastoma cells $(168,169)$. However, TGF $\beta$ inactivates NKG2D cells (170). Adoptive lymphocyte-therapy is a target of Treg cells/MDSCs. While the lymphoid cells (immune T cells, NK cells) kill tumor cells in vitro, they are disabled to do so in vivo. It appears that postoperative temozolomide and the EGFRvIII vaccine became the current standard therapy, except, tumor cells emerged without EGFRvIII expression, that recurred $(171,172)$.
Inflammatory events are essential in the induction and sustenance of glioblastoma. These events are supported by the alleviating effects of anti-inflammatory drugs on the clinical course of this malignancy. Non-steroidal anti-inflammatory drugs activate the genes NAG-1 (non-steroidal anti-inflammatory drug-activated gene), and growth and differentiation factor (GDF-15) in glioma cells. NAG-1 is usually silenced by dense methylations of its promoter; demethylation restores NAG-1 (173). NAG could stop migration and induce apoptosis (with troglitazone) of tumor (gastric cancer) cells (174). The distant TGF-relative, GDF, may be unpredictable: GDF-15 reduced susceptibility of HER2/ne $u^{+}$breast cancer cells to trastuzumab, and GDF-9 induced EMT in prostate cancer cells (175).

Inflammatory changes in the cerebrum bring about and sustain an incurable tumor of the highest lethality, and of chemoradiotherapy resistance. The most complex genomics of glioblastoma exhibit somatic mutations and loss of heterozygosity in its numerous oncogenes. The platelet-derived growth factor and VGF are up-regulated; tumor suppressors p53, RB and CDKN (cyclin-depedent kinase inhibitor) are deleted (176). An Achilles heal of this tumor is revealed by its epigenomics. The oncosuppressor microRNAs-487ab are down-regulated and the oncoprotective microRNA-502 and microRNA-532 are up-regulated. Two other microRNAs (miR-17-5p and miR-106a) are related to tumorigenesis and survival (177). Could the oncoprotective miR-502/532 be attacked by complementary siRNAs? Will targeted therapy (suppression of PDGF with imitanib, VEGF with bevacizumab, iNOS with mercaptoethyl-guanidine, cyclooxygenase with sulindac sulfide, or indomethacine, etc.) induce remissions, which could be maintained with the immunotherapeutic modalities (vaccines and adoptively administered immune T lymphocytes and LAK cells), which, however, unfortunately seldom if ever are supported outside the NIH/NCI (161).

Papillary carcinoma cells of the thyroid release inflammatory cytokines. The pathogenesis of Hashimoto's autoimmune thyroiditis and its consequences of B cell lymphoma or papillary carcinoma have recently been reviewed, but without being able to recognize the initiator(s) of these conditions (178). The customary chain of events is followed: DCs recognize thyroglobulins and thyroid peroxidases, instruct $\mathrm{CD} 4^{+} \mathrm{T}$ cells to engage $\mathrm{B}$ cells to produce antibodies. The expanding B cell population first is polyclonal, until after one MALT-like B cell clone emerges for monoclonal expansion. In the background, because of CTLA-4 (cytotoxic T lymphocyte-associated antigen 4) gene polymorphism, the inhibitor of autoimmunity becomes deficient. The autoimmune B cell clone shows some rearrangement of its IgM heavy chain locus and may undergo the translocation $t(8 ; 14)$ (q24;q32). Apparently no Treg clones rise to inhibit the proliferation of the autoimmune B cell clone $(178,179)$.

The fusion oncoprotein RET/PTC (re-arranged transformation papillary thyroid cancer) is formed and the MAPK signaling pathway is activated. Elevated levels of nitric oxide synthases (NOS) increased NO levels; vascular endothelial growth factor and its receptors VEGFR-1, -2, angiopoietin-2 and its receptor Tie 2 were overexpressed; so was the endothelin-1 pathway (180). The RET oncoprotein activates inflammatory genes within thyrocytes; these are the granulocyte/monocyte growth factor, IL-1 $\beta$, cyclooxygenase- 2 , chemokine ligands 2 and 20, IL-8 (chemokine ligand 8), chemokine receptor 4 (CXCR4), extracel- 
lular matrix-degrading enzymes and lymphocyte selectin genes, mRNAs and gene product proteins. $\mathrm{NF} \kappa \mathrm{B}$ and transforming growth factor $\beta$ (TGF $\beta$ ) levels are high and protect tumor cells from apoptotic death. The activated STAT pathway drives tumor cell replication and inhibits DC maturation, thus immature DCs establish a tolerant Th2-type internal environment (181). The presence of $\mathrm{CD} 4^{+} \mathrm{CD} 25^{+} \mathrm{FoxP} 3^{+}$tumor-associated lymphocytes allowed larger tumors and their locomotion to form lymph node metastases (182).

Further pecularities in autoimmune thyroiditis, the thyroid lymphoma and carcinoma are that: i) The background immunologic milieu of the host is Th1-type, so much so that the inducer $\mathrm{T}$ cell clone may turn malignant. The malignant $\mathrm{T}$ cell clone was $\mathrm{CD}^{-}$but $\mathrm{CD}^{+} \mathrm{CD}^{+}{ }^{+} \mathrm{TCR}^{+}$and chemokine-producer $\left(\mathrm{CXCR}^{+} \mathrm{CCR}^{+}\right)(183)$. In contrast, is it not so that the antibody producer B cell clone is the product of a Th2-type environment? ii) A combat of the reactive lymphocyte clones results in the appearance of many apoptotic cells (184). The question is who are the killers and who are the victims? iii) The inhibitory signals against autoimmunity derive from the CTLA-4 pathway of T (and B) lineage lymphocytes. However, the CTLA-4 pathway does not appear inactivated, even soluble CTLA-4 receptors circulate and are able to react with their ligands CD80/86 (185). Yet autoimmunity occurs and culminates in a $\mathrm{B}$ lineage lymphoma. There is an effort to block CTLA-4 with ipilimumab to induce autoimmunity against tumor cells (melanoma) masquerading for exemption and acceptance as self. However, in an environment of autoimmunity already in existence, papillary carcinoma cells are able to arise and prevail. These questions remain unanswered even in the best and most recent texts (186). The papillary carcinoma cells release the inflammatory cytokines (181). The situation in Greaves' autoimmune hyperthyroidism is better understood. There, autoantibodies to the overexpressed thyroid-stimulating hormone receptor (TDHR) and decreased Treg cells in number and function co-operate with IFN $\alpha$-producer plasmacytoid DCs that cause apoptotic death of the Treg cells (187). It is still unclear what inflammatory process activates the plasmacytoid DCs in the first place: may be an endogenous retrovirus?

Barrett's esophagus and esophageal adenocarcinoma. Barrett's esophagus (BE) is a premalignant condition consequential to chronic gastroesophageal reflux disease (GERD). BE may be viewed as an inflammasome, which harbors a chronic inflammatory process and the germline mutations of one or all three suspect genes: macrophage scavenger receptor-1 (MSR1, locus at 8p22); adenosquamous cell carcinoma-1 (ASCC1, locus at 10q21-22); and collagene triple helix repeat containing-1 (CTHRC1, locus at 8p22.1-24.22). MSR1 mutations are frequently of somatic, and occasionally of germ-line causation. MSR1 mutation leads to up-regulation of the cyclin D1 gene both in adeno-, and squamous cell esophageal carcinomas (CCND1) (188-190). The major inducer of the inflammatory reactions is deoxycholic acid (DCA); its effects on esophageal carcinoma cell lines (squamous cell carcinoma HET-1A, adenomatous carcinoma SKGT4) were studied in vitro. DCA induced IL- 8 in both, and IL- $1 \alpha \beta$ in the adenomatous cell line (191). The tribbles-homology-3 (TRB-3) gene (a gene controlling mitogen-activated protein kinase cascades) (191,192), was induced in the squamous, and silenced in the adenomatous cell line; it was suppressed in most other esophageal carcinoma cell lines in silico. It could be experimentally silenced by small interfering RNA (siRNA) preparations. Re-induction of the TRB-3 gene into the adenomatous cell line resulted in the release of $\mathrm{NF \kappa B}$, a cytokine known to activate inflammatory cytokines upon its intranuclear translocation $(190,191)$. In BE active are IL-6 and signal transducer and activator of transcription (STAT) (192,193), oxidative stress inducible NO synthase (194), cyclooxygenase-2 (195), and prostaglandins (196). In BE, inflammatory gene expression is associated with the activity of miRNA-375. Poor prognosis-associated lymphokines were IFN $\gamma$, IL-1 $\alpha$, IL-8, IL-21, IL-23. Co-expression of miRNA-375 improved the prognosis, so did expression of the pro-apoptotic and cell proliferation inhibitor annexin-1 (ANXA1), which was inhibited in most esophageal tumors (197).

In the benign stage of Barrett's esophagus, the artificial induction of intracellular acidification elicited the appearance of reactive oxygen species (ROS) followed by double-stranded DNA breaks. These were detected by immunofluorescence for histone $\mathrm{H} 2 \mathrm{AX}$ phosphorylation and were prevented by pretreatment with isothiocyanate-stilbene-disulfonate or acetylL-cysteine.

Non-repaired dsDNA breaks are considered to be precancerous events (198). MicroRNAs intervene in esophageal carcinogenesis. In comparison with healthy and cancerous tissues, down-regulated microRNAs in the cancer tissue are considered to be tumor suppressors lost, whereas up-regulated microRNAs are suspect cancer inducers. Elevated level of miR-21 in non-cancerous tissues of patients with squamous cell carcinoma, and reduced levels of miR-375 in cancerous tissues of patients with adenocarcinomas foretold adverse clinical course. This review describes the micro-RNA technology (isolation and quantification) in good details. The very valuable Fig. 2A of the cited article compares adenocarcinoma cancerous tissue and non-cancerous tissue levels (with a typographic error overlooked by the editors): in tumor tissue overexpressed were miR-21, miR-223, miR-192, and miR-194; underexpressed was miR-203. In Fig. 2B, squamous cell carcinoma tumor tissue overexpressed miR-21 and underexpressed miR-375. In Fig. 2A, miR-375 is not mentioned in connection with adenocarcinomas, but in the conclusion it is pointed out to be an adverse prognostic factor for BE-associated adenocarcinomas (199).

Pancreatic adenocarcinoma. Chronic pancreatitis leads to adenocarcinoma induction. In Romanian patients studied at Haţieganu Medical School in Cluj-Napoca (Kolozsvár), Transsylvania, TNF $\alpha$ and IL-6 are the dominant cytokines in that condition. In the malignantly transformed tissue, IL-8 and macrophage inflammatory protein-3 $\alpha$ appear in elevated levels (200). In tumorigenesis of the gastrointestinal tract, TGF $\beta$ first exerts anti-inflammatory and anti-oncogenic effects. Once a malignant tumor is generated, TGF $\beta$ becomes its supporter by promoting its invasion and metastases, and by subverting its stroma for the production of cytokines with paracrine circuitries to receptors of the tumor cell (201). For example, fibroblasts of the pancreatic cancer's microenvironment secreted neuregulin-1 for the phosphorylation and thus activation of Erb3 (epidermal growth factor receptor) and AKT (activated kinase transforming/ thymic, also known as protein kinase B) signaling pathways in the tumor cells. Erlotinib and the Erb3-directed mcab MM-121 shut down this pathway and halted the growth of pancreatic 
cancer xenografts (202). The sHH oncogenic pathway is active in pancreatic cancer cells. Here stem cell gene product proteins Nanog, c-Myc, Oct-4 (nanogs were the celtic people remaining eternally young, avian myelocytic leukemia oncogene, octamerbinding motif) drive tumor cell growth. A laboratory product small hairpin shRNA is inhibitory to Nanog. Natural products epigallocatechin-3-gallate and quercetin synergistically inhibited the $\mathrm{SHH}$ pathway and by overcoming Bcl-2- and XIAP- (X-liked inhibitor of apoptosis) promoted tumor cell death (203). A masterfully constructed cartoon depicts the oncogenic genome within pancreatic cancer cells and the cancer-supporting and inflammatory and immunoevasive activities (cyclooxygenase-, prostaglandin-, and VEGF-production, Treg cells, myeloidderived suppressor cells and tumor-associated macrophages) of the tumor's microenvironment (204). The tumor cell itself exerts effective immunoevasive maneuvers. Supernatants of pancreatic cancer cells inhibit CD4 T cell proliferation and migration, but induced (failed to inhibit) IFN $\gamma$ production. However, allowed CD69 ${ }^{+}$lymphocyte subset expansion (205). The cluster of differentiation marker CD69 characterizes most NK/NKT cells and as such it regulates CD17 lymphocytes in establishing Th-17 type immune environment (206). In imitating Treg cells for the neutralization of immune $\mathrm{T}$ cells, pancreatic cancer cells are able to express Fox 3 under the inducing effect of TGF $\beta 2$. When siRNA suppressed Fox 3 expression, the cancer cells secreted IL-6 and IL-8 (that were suppressed when Fox3 was expressed). Mimicking Treg, pancreatic cancer cells antagonized immune T cell clonal expansion (207).

The lectin galectin-3 is targeted by matrix metalloproteinase-7 (MMP-7, matrilysin). MMP-7 levels are high in the blood of patients with pancreatic adenocarcinoma. In mice STAT3 dictates the pace of pancreas adenocarcinoma cell divisions and MMP-7 expression $(208,209)$. The ZIP4 protein (zink transporter protein; Zrt/Irt-like protein: zink/iron responsive transporter) works with IL-6 and STAT in stimulating pancreatic cancer cell growth; silencing it with shRNAs inhibits cancer cell growth $(210,211)$. Of cancer cells growing in holo-, mero-, and paraclones, it is the holoclone stem cells that are most chemoresistant, express most of the stem cell genes and corresponding microRNAs and yield most of the tumor-initiating cells (212). In pancreatic ductal adenocarcinoma cells miR-155 targets and destroys the tumor suppressor p53-induced nuclear protein 1 (TP53INP1). Next, the tumor metastasis suppressor E1A-binding protein (EP300) is neutralized by miR-194, miR-200b, miR-220c and miR-429.

The tumor suppressors DPC/Smad4 (DNA-picked chromatin; signaling mothers against decapentaplegic; decapentaplegic $=$ TGF $\beta$ ligand $)$ are knocked out by miR-421 and miR-483. The miR-132 and miR-212 inhibit the binding and neutralizing the E2F protein by RB. The NFKB-repressing factor (NKRF) is neutralized by miR-301, thus liberating NFKB. The 'sprouty homolog' of drosophila, Spry2, is neutralized by miR-27a. The tumor suppressor inhibitor of growth protein ING4 is targeted for neutralization by miR-214. In contrast, those miRs that may act as tumor suppressors (miR-34, a p53 activator; miR-96, a K-ras inhibitor; miR375, a pro-apoptotic stimulator by inhibiting 14-3- $\zeta$ and down-regulator of Akt; miR-15a, a wingless drosophila Wnt/ $\beta$-catenin and fibroblast growth factor-7 inhibitor) (213) remain silent in the inflammatory carcinogenesis process within the pancreas. A detailed tabulation of up- and down-regulated microRNAs in pancreatic cancer with literature citations was made available (214), both references from M.D. Anderson Hospital $(213,214)$.

In the human genome, the promoter gene of the inflammatory cytokine IL- $1 \beta$ undergoes single nucleotide polymorphisms (215), that alone without a specific pathogen may initiate an inflammatory process. The stimulated IL-1 signaling pathway up-regulates the tumor promoters nicotinamide phosphoribosyl transferase and prostaglandin $\mathrm{H} 2$ synthase in human pancreatic cancer cells (216).

Oncogenesis in the pancreas shows how an inflammatory process can trigger a cascade of oncosuppressor gene silencing and oncoprotein activation, and that the DNA is a willing partner in the plot. It appears as if in an ancient cross-talk resumed, the RNA mobilizes its forces to rescue the DNA from a host threatened with an impending demise (vide infra).

Inflammatory breast cancer. Lymphangitic loco-regional spread of tumor cells results in the occlusion of lymph channels causing the 'peu d'orange' cutaneous edema. However, there is redness, warmth and pain. No infectious pathogens are present (negative stains and cultures). The host defensive reactions are dominated by scanty monocytic-lymphocytic infiltrates; no eosinophils, no granulocytes, no purulence. It appears to be an endogenously activated inflammatory process ('intrinsic inflammation') without any explicitly recognizable external pathogen. The breast cancer associated BRCA gene-defective cancer cells can not repair, but live with, dsDNA breaks. The repair enzyme polyadenosine diphosphate ribose polymerase [PARP, to be distinguished from PPAR, the peroxisome proliferator (vide infra)] would initiate ssDNA strand repairs. However, PARP inhibitors (olaparib, and the anti-inflammatory cordycepin) send BRCA-mutated breast cancer cells through the apoptotic death pathway.

In a murine breast cancer model, leptin activated the Notch signaling pathway and IL-1 and VEGF/VEGFR-2 overexpression (217). The thymic stromal lymphopoietin (TSLP, a IL-7-like type 1 inflammatory cytokine signaling through TSLP-R in $\mathrm{CD}^{+}$cells and thus inducing IL-10 and IL-13 production for a Th2-type host immune environment) promotes the progression of breast cancer cells both by lymphatic and vascular routes. Inactivation of TSLP in a mouse model, resulted in the cessation of all tumor metastases (218). The inflammatory cytokine, TNF $\alpha$ promotes the growth of HER2/neuT breast cancers in mice. Deprivation of TNF $\alpha$ resulted in disarranged tumor vasculature and conversion of Th2-type to Th1-type inner immune environment (unexpected, as TNF $\alpha$ with IFN $\gamma$ are classical inducers of the Th1-type inner environment) (219). The relevance of these pathways to human breast inflammatory cancers is unknown. However, TNF $\alpha-308$ polymorphism comes with reduced breast cancer incidence in Caucasian women (220). The polycomb protein, zeste homolog-2 (EZH-2) is overexpressed in human inflammatory breast cancer cells with cell cycle progression resulting. Patients with zeste-expressor inflammatory breast cancer cells had short survivals (221). ErbB1/2 overexpression is also frequent in inflammatory breast cancer cells. The patented lapatinib analog GW583340 induced cytoplasmic ROS (vide infra) formation with apoptotic cell deaths following; these effects were reversed by superoxide dismutase (SOD). Inflammatory breast cancer cells with elevated levels of SOD and glutathione resisted apoptotic deaths due to no $\mathrm{H}_{2} \mathrm{O}_{2}$ (ROS) 
formation upon treatment with the lapatinib analog or paraquat (222).

The infiltration of human breast cancers with $\mathrm{CD}^{+}$ Tlymphocytes is correlated with better prognosis (223). However, a subclass of $\mathrm{CD}^{+} \mathrm{T}$ cells, appears to promote lymphangitic spread of human breast cancer cells toward regional (axillary) lymph nodes. These breast cancer cells undergo EMT. The lymphocytes are of the ILEI subclass (interleukin-like EMT inducers). This subclass of T lymphocytes expresses TNF $\alpha$ and TGF $\beta$ and needs the co-operation of mutated Ras and Snail oncoproteins in the tumor cells (224). This author proposed the terminology of traitor/transforming $\mathrm{T}$ cells $(\mathrm{T} / \mathrm{T} \mathrm{T})$ for this subclass of lymphocytes (225). However, no matter how attractive it is to presume it, it is not known if the T/T T subclass of lymphocytes induces the inflammatory type of breast cancer. In cooperation with chemokines CCR4/CCL22, DC-activated and tumor antigen-specific CD4 ${ }^{+}$Treg cells infiltrate breast cancers and contribute to the cancer's immunosuppressive process (226a).

Gene sequences related to the mouse mammary tumor retrovirus (Bittner virus) occur in human breast cancers (226b), but further proof is needed for the causative, and/or inflammation-inducing effects of these agents. Many human tumors (teratocarcinomas, melanoma, ovarian adenocarcinoma, and malignant lymphomas express endogenous retroviruses, but without solid proof for etiologic connections (225) (vide infra at Kaposi's sarcoma).

The Michigan Cancer Foundation's human breast cancer cell line MCF-7 contains cells of the luminal cancer stem cell markers (ESA, CD44 ${ }^{\text {hi }}$ hyaluronate-binding pro-metastatic adhesion molecule, $\mathrm{CD} 24^{\mathrm{lo}}$ selectin-binding adhesion molecule, $\mathrm{CD}_{133^{+}}$, and chemotherapy-resistance Drug $\left.{ }^{\mathrm{RES}} \mathrm{MCF}-7\right)$. Numbpeptide-activated cytotoxic T cells killed CD $44^{\mathrm{hi}} / \mathrm{CD} 24^{\mathrm{lo}}$ cancer stem cells. Patients with breast cancer may benefit from treatment with Numb-specific adoptive lymphocyte infusions, or vaccination with Numb peptides (Numb, Notch oncoprotein antagonist, from drosophila) (227). However, if $\mathrm{Numb}^{+}$cells are eliminated, its antagonist Notch may prevail. Further, in breast cancer cells Numb protects pro-apoptotic p53 from degradation (cited in ref. 228).

The M.D. Anderson team recognized a chain reaction with the pro-inflammatory TNF $\alpha$ activating IKK $\beta$ (I $\kappa$ kinase), which then inactivated the cancer suppressing complex of TSC1/TSC2 (tuberous sclerosis), which usually acts by holding down mTOR. $\mathrm{NF} \kappa \mathrm{B}$ gets liberated in the process and transits from cytoplasm to nucleus to activate inflammatory and potentially oncogenic genes $(229,230)$. A comparison of the genomics of inflammatory and non-inflammatory breast cancers revealed overexpression of immune system and mTOR pathways in inflammatory breast cancers (231). The Michigan Comprehensive Cancer Center found overexpressed EGFR and disabled p53 genes. More significantly, the RhoC (Ras homolog) GTPase GTP-binding protein gene was overexpressed in $90 \%$, whereas the Wisp3/ LIBC tumor suppressor gene (Wnt-induced secreted protein, from wingless drosophila; lost in inflammatory breast cancer) was deleted in $80 \%$ of the specimens examined (232). In France, those inflammatory breast cancer stem cells that overexpressed aldehyde dehydrogenase (ALDH) metastasized most frequently. Graphs show high rate of mortality in $\mathrm{ALDH}^{+}$inflammatory breast cancers (233). However, non-inflammatory breast cancers expressing ALDH also run an adverse clinical course.
Breast cancer stem cells' DNA is highly oncogenic showing 100 to 1,000-fold increased tumorigenicity in xenografts, more than that of the general cell population of the MCF-7 (Michigan Cancer Foundation) established cell line (vide supra). The stem cell phenotype is $\mathrm{ESA}^{+} \mathrm{CD} 44^{+} \mathrm{CD} 24^{+/ l o w}$ (epithelial-specific antigen). The oncogenic stem cell DNA is highly chemoresistant. The cell line under the effect of miR-27b overexpresses the drug-metabolizing enzyme CYP1B1 (cyclophilin). The active miR-21 targets the mRNAs of tumor suppressor genes tropomyosin (TPM1) and PDCD4. The tumor promoter gene pleomorphic adenoma (PLAG) is targeted by miR-200a and miR-224 and the activities of these miRs are curtailed in the oncogenic stem cells (234). A report names six microRNAs that are overexpressed (miR-335, miR-337-5p, miR-451, miR-486-3p, miR-520a-5p, miR-548d-5p) and seven microRNAs (miR-1a, miR24, miR-29a, miR-30b, miR-320, miR-342-5p, miR342-3p), that were down-regulated in inflammatory carcinomas of the breast. However, the exact genomic origins of most of these miRs have not as yet been pinpointed (235). Inflammatory cytokines IFN $\gamma$, IL-6, LPS endotoxin, and poly(I:C) activate microRNA-155 in breast cancer cells; miR-155 then activates the STAT and JAK (Janus kinase) 'cell survival' pathways connecting inflammatory immune stimulation with the inception of oncogenesis (236).

The inflammatory COX-2 expression appears early: already active in ductal carcinoma in situ (DCIS), catalyzing arachidonic acid to prostanoids. Its inducer is 12-myristate 13-acetate that could be blocked with the flavonoid apigenin $(237,238)$.

The very rare incidence of anaplastic large cell lymphoma (ALCL) beneath silicone breast implants occurred in women receiving the implants for cosmetic reasons (not after mastectomies for breast cancers). The lymphoma cells are $\mathrm{ALK}^{-\mathrm{CD}} 2^{+}$ $\mathrm{CD}^{+/-}{ }^{-\mathrm{CD}} 30^{+} \mathrm{CD} 20^{-} \mathrm{CD} 45^{-}$granzyme $\mathrm{B}^{+} \mathrm{EBV}^{-} \mathrm{T}$ cells of defective $\mathrm{T}$ cell receptors. The lymphoma cells express the anti-apoptotic myeloid cell leukemia-1 protein (MCL-1) controlled by microRNA-29 (it suppresses MCL-1 expression). In ALCL, low miRNA-29 levels allow high expression of MCL-1 (providing protection against apoptosis). It is presumed to be induced by unidentified cytokines from histiocytes reactive to silicone particles (239-241).

Non-silicone implant-related $\mathrm{ALK}^{+}$anaplastic large cell lymphomas overexpress the anti-apoptotic MCL-1 protein (while Bcl-2 is silent). The MCL-1 protein is under the control of microRNA-29a: in ALK'ALCL miR-29a levels are low due to methylations of the miR-29a gene, thus MCL-1 is liberated. In this lymphoma the nucleophosmin (NPM) and ALK genes fuse: $\mathrm{t}(2 ; 5)(\mathrm{p} 23 ; \mathrm{q} 35)$, thus creating the NPM/ALK oncoprotein (NPM is nuclear phosphoprotein B23 numatrin). The NPM/ ALK oncoprotein activates the PI3K/Akt and mTOR pathways; the reaction chain continues in the constitutive activation of signaling from sHH/GLI (sonic hedgehog; glioma-associated oncogene homolog) $(242,243)$.

Colonic polyps and adenocarcinoma. The classical 'Vogelgram' describes the sequential gene deletions, mutations and fusion oncoproteins that lead to the transformation of colonic polyps into adenocarcinomas (244). In this scheme, peroxisome proliferator-activated receptor $\delta$ (PPAR $\delta$ ), a potential tumor promoter, responding to its natural ligands of fatty acids prostacyclins/prostaglandins and active in inflammatory processes, 
is suppressed by the product of the adenomatous polyposis coli gene (APC), whose tumor suppressor pathway consists of APC/ $\beta$-catenin/T cell factor-4 (TCL-4). This pathway is lost to mutations and deletion at $5 \mathrm{q} 21$. In the healthy tissues, the APC gene product protein inhibits the complex formation between $\beta$-catenin and TCL-4. Among others (c-Myc), PPARd is another target of the $\beta$-catenin/TCL-4 complex (the PPAR $\delta$ promoter contains the TCL-4 binding sites). PPAR $\delta$ mRNA levels are high in colorectal tumor tissues, but suppressed by the functional APC. Deleted (or lost to mutation or silencing) are at $17 \mathrm{p}$ the proapoptotic p53, the Waf1/CIP1 (wild-type p53 activated fragment; cycline kinase inhibitory protein) at $21 \mathrm{p}$, the Dickkopf, the Wnt antagonist, the colon cancer suppressor gene (DCC) at 18q21, the $\mathrm{nm} 23$, the non-metastatic gene at $17 \mathrm{q} 21$, and the mutated colorectal cancer gene (MCC) at 5q. Point-mutated is at $5 \mathrm{q}$ in its codon 12 the K-ras with activation of c-Myc at 8q24. Loss of heterozygosity at 5q21 involves the APC/MCC genes (244-247).

The colonic polyps are 'inflammasomes' expressing great histiocytic, leukocytic and mast cell activities; CXCL12 (stromal derived factor, chemokine) switches from IL-10 to IL-17 and from anti- to pro-inflammatory Treg cells. The advancement of adenomas (polyps) into adenocarcinomas is through an 'inflammatory phenotype' characterized by attraction of leukocytes and macrophages into these lesions by IL-8. Knock-out of the pro-apoptotic p53 gene secures cell survival. Paracrine neovascularization is elicited and activation of the cell motility processes by chemokines (CCL20-to-CCR6; up-regulation of cyclooxygenase- 2 by CXCL1) initiate the process. Activation of 'cell survival pathways' (STAT, MAPK, PI3K/Akt) overcomes the opposing forces of MyD88 and IL-18 driving the cell to the stage of no return, that is, the switch of stem cell genes away from differentiation to immortalization (recognized clinically as 'malignant transformation'). The proinflammatory genes involved, many TLRs (especially TLR4), and chemokine genes originating from the innate era, are identified: CXCL1, CXCL2, CXCL3, IL-8, CCL5. CCL19, CCL20, CCL21, CCL23, CCL5; and inflammatory $\mathrm{iNOS}^{+}$macrophages are activated (248-253). However, Hanahan and Weinberg omitted inflammatory contributions from the list of carcinogenic events. Mantovani et al supplemented trait number 7: inflammatory carcinogenesis (254-256).

At the Semmelweis University, Budapest, Hungary, a major engagement is underway for the elucidation of key mechanisms in colonic carcinogenesis (257). What goes wrong in the lymphoid follicles receiving stem cell supplements for the regeneration of the colonic mucosal lining? The colonic epithelial cell layer is shed and regenerated by an upward flow of new cells in the crypts in rapid successions. The gut-associated lymphatic tissue (GALT) is dispersed as isolated lymphatic follicles (ILFs). ILFs receive bone marrow-derived stem cells (BMDSC) through their blood and lymphatic vascularization. In ILFs, the resident multiple lymphoid elements, dendritic cells, and syncytia of myofibroblasts encounter and interact with the arriving BMDSCs. The ILFs preserved innate immune faculties and as such, are immediately reacting through TLRs. For the repair of mucosal bowel wall damage, ILFs initiate the mobilization of epithelial cells in the crypts $(257,258)$. The contact between ILF cells and epithelial cells is through the Wnt-Lgr5 (wingless in drosophila; leucin-rich repeat containing $\mathrm{G}$ protein-coupled receptor 5) pathway. Lgr5 is overexpressed in metastatic colorectal cancer cells. These cells undergo EMT, either by mobilizing their own Twist and Snail gene product proteins, or by fusing with subverted tumor-associated macrophages (257-260). The colonic mucosa continually is at an inflammatory alert $(252,256)$, that facilitates the switch diverting from the regenerative function in the stem cells, to the process of immortalization (malignant transformation), upon repeated intake of nutritionally introduced chemical toxins.

The commonly present LPSs and TNF $\alpha$ readily activate $\mathrm{REL} / \mathrm{NF} \kappa \mathrm{B}$. The gene of this ancient transcription factor was discovered in a strain of the avian reticuloendothelial virus (c-rel $\rightarrow \mathrm{v}-r e l)$. NFאB up-regulates TGF-R $\beta I I$. In colon

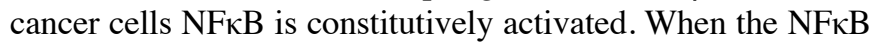
up-regulator I $\mathrm{B}$ kinase $\gamma$ was knocked out by iRNA, tumor cell xenografts regressed $(261,262)$, The tumor suppressor oncostatin M (OSM) is silenced early in colonic polyps and colon carcinomas. When active, OSM reduces tumor cell proliferation, induces differentiation or apoptosis. It is neutralized by DNA methylation, or histone deacetylation. Methyl-transferase inhibition (5-aza-deoxycytidine) and histone re-acetylation (deacetylase inhibitor, trichostatin) re-activated OSM in colon cancer cell lines (263). The microRNA-137 acts as a colon cancer cell suppressor, but early in the process of carcinogenesis, $\mathrm{CpG}$ islands of its gene are hypermethylated and thus silenced (264). In contrast, microRNA-21 interacts in a mutual stimulatory way with inflammatory events (IL-6, IL-8, IL-12a, the tolerogen IL-10, and with the genotoxic NOS2). These actions spelled out a high inflammatory risk score and cancer-specific mortality (265). In mice, inflammation in the bowels mediates the methylation and silencing of the polycomb target genes (for example, lysine 27 on histone 3 was trimethylated) (266). In contrast, reacetylation of histones by histone deacetylase inhibitors (DHDACI, vide supra) could induce intrinsic (mitochondrial) apoptosis of colon cancer cells (267). In Shanghai, P.R. China, a genetically engineered adenovirus delivers the XIAP-associated factor 1 gene (XIAF), which neutralizes the anti-caspase activity of the apoptosis inhibitor XIAP (X-linked inhibitor of apoptosis). The TNF-related apoptosis inducing ligand (TRAIL) is then administered. In colon cancer cell xenografts in mice thus treated, the entire anti-apoptotic machinery (XIAP, c-IAP-2, survivin) was neutralized, and the xenografts were destroyed (268).

The colon is the battlefield between bacteria and bacteriophages and bacteria and the host (164). Its genomics/proteomics and epigenetics have gone through a very long evolutionary history. Add the uninvited guest, the human polyomavirus JC, whose $\mathrm{T}$ antigen inactivates $\mathrm{p} 53$, while it liberates $\beta$-catenin for intranuclear entry for interactions with proto-oncogenes. It is present in 271/766 (35\%) of human colon cancers (269). Now emerges the enterotoxigenic Bacteroides fragilis, as a major contributor to colonic carcinogenesis, according to data emanating from the Johns Hopkins' Cancer Institute (270).

The $B$. fragilis enterotoxin cleaves E-cadherin and thus activates the $\beta$-catenin/sHH/Wnt cascade. The enterotoxin is neoangiogenic. Colonic mucosal epithelial cells proliferate due to the activation of the STAT/MAPK cascades. Activated $\mathrm{NF \kappa B}$ and c-IAP2 (X-linked inhibitor of apoptosis) protect these cells from apoptotic death. The proliferating mucosal cells exude IL-8, an activator of NFkB, cyclooxygenase-2 and prostaglandin. The bacteroides enterotoxin re-arranges the 
cytoskeleton of the mucosal epithelial cells. In the process, protooncogenes K-ras and c-myc are activated. Further, the human homolog of the drosophila gene 'legless', the B cell lymphoma gene BCL9 becomes activated. This gene is an activator of the $\beta$-catenin/Wnt cascade. The major inhibitor of this cascade, the dickkopf-1 gene product protein is not produced due to the silencing of the dickkopf-1 gene (or its promoter). Some other dickkopf genes (dickkopf-3, -4) are up-regulated; their gene product proteins are neoangiogenic, but the Dickkopf-4 protein is inhibitory to the $\beta$-catenin signaling pathway. Other cyto- and lymphokines activated by the bacteroides enteroxin-stimulated colonic mucosal cells are IL-6, IL-8, IL-10 and TGF $\beta$. Cytidine deaminase, TNF $\alpha, \mathrm{NF} \mathrm{B}, \mathrm{IL}-4$ and IL-13 somehow eliminate 'the guardian of the genome', p53, in the colonic mucosal cells. Reactive $\mathrm{T}$ lymphocytes liberate $\beta$-catenin for intranuclear entry. Reactive lymphocytes express $\mathrm{T}$ cell factors: TCF-1 promotes, TCF-4 inhibits the Wnt/sHH cascade. Colon cancer cells often express the Fas ligand $\left(\mathrm{FasL}^{+}\right)$, thus $\mathrm{FasR}^{+}$immune $\mathrm{T}$ cells upon contact with these cancer cells die apoptotic death (extensively reviewed in ref. 62 and original references cited in ref. 270). IL-17 appears. This lymphokine may act as a tumor suppressor, when it activates DCs and NK cells; however, if it activates vascular endothelial cells, granulocytes and macrophages, it acts as a tumor promoter (70). In human $\mathrm{CpG}$ island methylator geno-phenotype colon cancer tissue (proven by assaying eight genetic markers), the effect of lymphocytic infiltrates on survival was favorable. The lymphocytes assume Crohn-like, peritumoral, intratumoral periglandular reactions, and tumor-infiltrating patterns (shown in microphotographs). The tumor-infiltrating pattern was associated with significantly improved tumor-specific and overall survival (271).

Further recent reviews of carcinogenesis in ulcerative colitis explain the genomics and proteomics of that condition, the role of IL-13 in the activation of the cell survival STAT pathway (272), but without being able to recognize the ultimate inducing agent(s) (recently reviewed in ref. 62). The highly methylated genes in the inflamed mucosa are the promoter of E-cadherin (CDH1), the transmembrane protein containing epidermal growth factor hyperplastic polyposis (TPEF/HPP), the glial cell line-derived neurotrophic factor (GDNF) and the myoblast differentiation genes (273). Of these genes, CDH1 and HPP exert tumor suppressive functions. This author is impressed by one rare side effect of ipilimumab: 'the hemorrhagic colitis' syndrome. A middle-aged male patient D.L. with metastatic melanoma was sent to the NIH/NCI clinics (Bethesda, MD) to receive a then investigational mcab ipilimumab. While he experienced a better than $50 \%$ partial regression of his melanoma, he developed 'hemorrhagic enterocolitis' with perforation and died in septic shock. Even though he received corticosteroids to alleviate his 'autoimmune enterocolitis', at postmortem examination (performed by Dr László Karai), the ulcerated intestinal tract, especially the colon, was infiltrated by lymphocytes (unidentified as to clonality) (unpublished data). The inflammatory reactions in ulcerative colitis are of internal autoimmune causation without an external pathogen. Autoimmune diseases of the gastrointestinal tract involve different organs (pancreatitis with ulcerative colitis) (274). Adenocarcinomas and primary lymphomas of the entire gastrointestinal tract, especially those of the colon with ulcerative colitis, are of autoimmune inflammatory causation (275).
In the non-polyposis colon cancers, Lynch syndrome, inducer genes are named after their ancestors discovered in yeast cells (276): postmeiotic segregation (PMS), mutated S and L homolog (MSH, MLH) (277). In the MSH2-associated Lynch syndrome, the epithelial cell adhesion molecule (EpCAM) gene is also deleted $(278,279)$. In Lynch syndrome, the oncogenic DNA is repaired mismatched. Mismatched repaired DNA proved to be quite viable in the highly malignant transformed cells. Cancers due to germ-line gene mutations may not need much inflammatory stimuli. However, the sporadic mucinous colon cancers arising in hypoxic, inflamed microenvironment are commonly right-sided, multifocal and express the PMS. These mucinous colon cancers are infiltrated by lymphocytes, and express PMS2 gene mutations. In a mouse model, the histone deacetylase inhibitor suberoylanilide hydroxamic acid (SAHA) decreased peritumoral colitis and NFKB levels (280).

The intestinal tract suffers from protracted inflammatory processes in ulcerative colitis, Crohn's disease and in nonhealing rectal fistulas-fissures (281-283). Polymorphisms of the IL-6 genes, epigenetic methylations of the CpG islands of the WTp53 and KRAS2 genes translate into the CIMP (CpG island methylator phenotype) (vide supra). Oxidative-induced DNA breaks initiate and hypoxia-induced inflammation sustains the growing tumor (284-286).

Dietary anti-oxidants ( $\alpha$-tocopherol; $\beta$-carotene, the carotenoid, lycopene) and anti-inflammatory agents, aspirin or ibuprofen) are claimed to reduce the rate of inflammatory mutations. Lycopene and tocopherol decreased the rate of p53 mutations; carotens decreased the rate of KRAS mutations. In women, $\alpha$-tocopherol was protective to p53 against CIMP, whereas in men $\alpha$-tocopherol was associated with increased mutation rate in these situations. Only in GG IL-6 genotype did high tocopherol levels reduced risks of p53 and KRAS mutations (287).

Prostate cancer. Apart from vertically transferred somatic (acquired), or germ cell mutations (in the minority of cases), this tumor has long been suspected to originate with somatic gene mutations against the background of a chronic inflammation (in the majority of cases). For example, if Mycoplasmataceae are carcinogenic by activating the vav oncogenes (vide supra), could a genitourinary mycoplasma infection be inductive to prostatic adenocarcinoma? Prostatectomy specimens frequently yield (35\%) Propionibacterium acnes, or corynebacteria, or nanobacteria (288-291), but preceding genitourinary mycoplasma infections in the prostate might have been unrecognized. Calcifications in cancerous breast or prostate tissues may indicate reactions of hydroxyapatite and nanobacteria, if nanobacteria as true life forms really existed on Mars and were transferred to Earth by meteorites (292). As to genetic predisposition and inflammation, the deletion of glutathione S-transferase gene in combination with the histologic picture of 'proliferative inflammatory atrophy' lead to the precancerous intraepithelial neoplasia (293).

Mice transgenic for vav3 overexpression (vide supra) in the prostate develop $\mathrm{NF \kappa B}$ - and $\mathrm{PI} 3 \mathrm{~K}$-driven prostate cancers by 3 months of age. These cancer cells also expressed androgen receptor (AR) overactivity. The cancerous glands showed hypervascularity and heavy lympho-mononuclear cell infiltrates. Up-regulation of HER2 and down-regulation of PTEN 
occurred. When vav3 is activated in the prostate, it may positively interact with the AR, or it may stimulate the growth of prostatic cancer cells independently from AR by activating the PI3K/Akt 'cell survival' pathway $(294,295)$. The human $v a v$ proto-oncogene was mapped to $19 \mathrm{p} 12-12.2$ (137). Vav3 expression was significantly elevated in androgen-independent human prostate cancer cells. A siRNA complementary to vav3 mRNA inhibited the growth of both androgen-dependent and independent human prostate cell lines. Molecular inhibitors of PI3K were also effective in inhibiting the growth of $v a v 3^{+}$prostate cancer cells (296). These authors speak of 'non-bacterial prostatitis' (not to misread as 'nanobacterial'), but do not state if attempts at culturing mycoplasma from the precancerous gland were actually attempted. These authors succeeded in documenting vav3 oncogene activation in $81 \%$ of the human breast cancer specimens. Vav activated estrogen receptor expressions (297). These studies done at Mount Sinai Hospital, New York and at Albert Sabin Way, Cincinnati, OH, are entirely comparable with similar experiments conducted at the University of Miami's Miller School of Medicine $(295,296,298)$. Here, the Rho GTPase guanine nucleotide exchange factor Vav3 was found markedly increased in androgen-independent human prostate cancer cells. The stimulation of AR by Vav3 protein was active in the presence of subnanomolecular androgens. Thus, Vav3 could maintain prostate cancer cell growth in patients receiving androgen deprivation therapy, which is seldom absolutely complete. Intact pleckstrin strands are KSTR amino acid strings (lysine for $\mathrm{K}$, serine for $\mathrm{S}$, threonine for $\mathrm{T}$, arginine for $\mathrm{R}$, in the substrate of leukocyte kinase $\mathrm{C}$ of leukocytes and platelets) obligatory to the activity of Vav3 (298).

A pro-inflammatory condition in the pre-carcinomatous/ carcinomatous prostate is consequential to the lipooxygenase product, eicosatetraenoic acid (5-oxo-ETE). $\mathrm{H}_{2} \mathrm{O}_{2}$ stimulated the increase of 5-oxo-ETE, nicotinic acid adenodinucleotide phosphate (NADP) and glutathione disulfide. Glutathione reductase-dependent generation of NADP was blocked by N-ethylmaleimide (NEM). 5-oxo-ETE exerted strong chemoattraction toward granulocytes. Granulocytes further released inflammatory cytokines. Arachidonic acid and calcium ionophore induced self-stimulation of granulocytes by 5-oxo-ETE release, that could be blocked by NEM (299). Thus, these chemical reactions could induce prostatitis without a pathogen. A macrophage inhibitory cytokine-1, member of the TGF-family prostate-derived factor is claimed to inactivate suppressor genes and activate oncogenes (300).

Two genes held highly suspect in promoting inflammatory carcinogenesis in the prostate were the ribonuclease $\mathrm{L}$ (RNASEL) and the MSR1 genes. Further, the Toll-like receptor family genes and the cyclooxygenase gene have been under suspicion. Finally, single nucleotide polymorphism (SNP) of some lympho- and cytokine genes was considered to be contributory to inflammatory carcinogenesis of the prostate: IL-1 $\beta$, IL-6, IL-8, IL-10, and the TNF family genes. SNP in four inflammation pathway genes IL-4, IL-6; posttranscriptional gene silencing (PTGS2); and STAT3 were most significantly associated with inflammatory carcinogenesis in the prostate. Aggressive prostate cancers were associated with SNP in AKT1, PIK3R1 (phosphoinositide-3-kinase regulatory subunit 1) and STAT3 genes (301). (Prostatic intraepithelial neoplasia surrounded by heavy inflammatory infiltrate emerges as one of the most adverse prognostic factors. Rider JC, AACR Press release, Sept 27, 2011).

This most versatile tumor may use nitric oxide (NO) for its cells' survival. The plasma membrane molecule carboxypeptidase-D (CPD) obtains arginine from extracellular sources. Arginine is converted to NO intracellularly. Low doses of testosterone or prolactin up-regulate CPD. Through the arginine pathway more NO was generated; these tumor cells gained resistance to apoptotic death (302). NO suppresses Snail and activates Raf-kinase (rat fibrosarcoma) inhibitory protein (RKIP), thus inhibiting EMT of tumor cells (303). The prostate cell genes stearoyl CoA desaturase 1 (SCD1) and the insulin-induced gene 1 (INSIG1) may engage proto-oncogenes, the mesenchymal differentiation inducer gene MAF (musculoaponeurotic fibrosarcoma, MAP) and the Notch receptor ligand jagged (304), thus statins acting on SCD1, and metformins acting on insulin-like GF genes may suppress prostate cancer cells. Some prostate cancer cells de-differentiate toward bone marrow mesenchymal stem cell (305). Promoters of EMT are, among others, the loss of tumor suppressor genes p53 and PTEN (306); $\beta$-microglobulins; $\beta$-catenin induced by HIF-1 $\alpha$; ester-12 myristate 13 -acetate; TGF $\beta$; VEGF; and growth and differentiation factor 9 (GDF-9). Estrogen receptor $\beta(\mathrm{ER} \beta)$ expression is suppressed in aggressive high Gleason grade prostate cancer cells. The ligand to the ER, $5 \alpha$-androstane-3 $\beta$-17 $\beta$-diol, maintains the epithelial cell phenotype. Hypoxia and TGF $\beta$ suppress ER. Active ER suppresses HIF- $1 \alpha$ and VEGF-A. In the absence of ER activity, VEGF-A (neurophilin-1) promotes Snail-1 in the nucleus, which activates vimentin and all other genes to encode the mesenchymal phenotype (307). Prostate cancer cells undergoing EMT metastasize. Most circulating tumor cells display EMT phenotype (308a). Hypoxia, TGF $\beta$ and VEGF are notoriously active in the inflammasomes. The inflammatory oncoprotein NFKB activates the snail oncogene; the Snail oncoprotein is a major inducer of EMT. The proteasome inhibitor NPI-0052 (nonapeptide inhibitor salinosporamide A from marine actinomycete Salinospora tropica), and the NFKB inhibitor DHMQ (same as TMDQ, dimethyl or trimethyl-1,2-dihydroquinoline) repress Snail and reverse the process of EMT (308b).

MicroRNAs miR-143 and miR-145 inhibit EMT and bone metastases; however both miRs are down-regulated in high Gleason grade tumors and thus are inhibited to act (309). In aggressive prostate cancers, miR-221/222 canceled the mRNAs-to-protein translation of p27 (CDKN1B), and p57 (CDKN1C), thus cell growth suppressive cyclin-dependent kinases were silenced. The miR-16 can arrest the cell cycle in $\mathrm{G}_{0}-\mathrm{G}_{1}$ by negatively regulating (suppressing) HMGA1 (high mobility group) and CAPRIN (cytoplasmic activation/proliferation-associated protein) genes (310). 'The oncogene from hell', c- $m y c \rightarrow c-M Y C$, can be silenced by miR-145, except that miR-145 is rendered non-functional in aggressive tumors. Some c-MYC supportive miRs (miR-363, miR-92a, miR-20b, and miR-18b) are up-regulated. Three genes up-regulated in aggressive prostate cancers (Gleason $>8$ ) are CCNA2 (cyclin-A2), CDCA5 (cell division cycle-associated) and KIF23 (kinesin family, for rolling movement of the cell nucleus). The two miRs (miR-145 and miR-331-3p) negatively regulating these genes normally, are silenced. When ectopically re-expressed in prostate cancer cell lines, these two miRs exerted growth inhibitory effects (vide infra) (311). 
The TMPRSS2-ERG gene fusion at locus 21q22 takes place early in prostate cancer cells (transmembrane protease serine 2; ets-related gene; ets E26 avian erythroblastosis retrovirally acquired cellular-to-viral oncogene: c-ets $\rightarrow \mathrm{v}$-ets) (312). Androgen-signaling brings these two genes into proximity, thus inducing their fusion (313). Altogether, the untranslated 5'UTR region of gene TMPRSS2 (21q22) may fuse with ERG (21q22), ETV1 (7p21), SLC45A3 9 (solute carrier gene) and with HERV-K, the human endogenous retrovirus gene, that in extravillous cytotrophoblasts exerts immunosuppressive properties in the defense of the fetus $(314,315)$. It is the ERG element that dictates the malignancy, even when not fused: patients with increased copy number of the ERG gene in chromosome 21 have a high chance for relapse after radical prostatectomy (316). High expression of HSP-27 encoded from 7q11.23 independently from ETS gene rearrangement (vide supra) predicted high risk disease (Gleason >8) and poor prognosis (317). ETS1, ETS4 gene expressions are responsible for anchorage-independent high proliferative tumor cell growth (318).

Another retroviral genomic sequences (and mature virions, in this case) had allegedly existed in some human prostate cancer cells, both in the stroma and in the tumor cells. This is the xenotropic murine leukemia virus-related gammaretrovirus (XMRV). The chain of events is such, that TNF $\alpha$ activates $\mathrm{NF} \kappa \mathrm{B}$, which activates XMRV. Herpesviruses are known to activate retroviruses (reviewed in ref. 108) and in the human prostate EBV latent membrane protein-1 (vide infra) also activates XMRV. The p65/RelA (reticuloendothelial) component of NFkB attaches to two sites $\kappa \mathrm{B}-1$ and $\kappa \mathrm{B}-2$ in the UT3 region of the long terminal repeat of XMRV. The wild-type $\mathrm{XMRV}$ replicates in human prostate carcinoma cell lines, in a Burkitt's lymphoma cell line, and in an EBV-immortalized cell line (319). However, strong new evidence indicates that human prostate cancer xenografts passaged in nude mice picked up two murine proviruses (PreXMRV-1 and 2) which recombined to produce replicating XMRV retroviral particles (320). Picking up murine endogenous retroviruses by human tumor xenografts was observed before (321). The role of XMRV in inflammationinduced human oncogenesis, or in chronic fatigue syndrome, appears to have been negated $(320,322)$. Under these circumstances, it remains a tantalizing puzzle how positive results of viral isolations and serological evidence of human infection with the XMRV could possibly be reported. (High risk 'lethal prostate cancers' xenografted in immunosuppressed mice revealed the well known mutations of the TP53 gene and large numbers of highly individual non-germ-line somatic mutations, 'hypermutations', in DLK2, 8 -like homolog, GPC6, glypican-6, and SDF4, stromal cell-derived factor 4, genes; androgen suppression-resistance occurred in Wnt mutated tumors. The individual mutations continued to evolve in the xenografts. (Kumar A, et al, press release on Sept 26 from Fred Hutchinson Cancer Research Center, Seattle, WA).

Finally, the B cell-specific Moloney mouse leukemiasarcoma viral genome's insertion site BMI1 in the human genome remains a point of interest, inasmuch as this site is involved in the activity of stem cells with (or without) the oncogenic activities of the Sox oncoprotein (SRY-related high mobility box family transcription factor; sex-determining region in $\mathrm{Y}$ chromosome, vide infra). The BMI1 protein is a transcriptional repressor of the polycomb group gene family.
The immunoreactivity of the BMI protein is in the cytoplasm, where it acts as a negative regulator of the cell cycle (Ink4a/ Arf, inhibitor cyclin-dependent kinase 4; alternative reading frame), thus cell proliferation. BMI1 is active in stem cells and in several tumors, including gastric and prostatic adenocarcinomas $(323,324)$.

Both stromal and malignant epithelial cells in the prostate gland express keratinocyte growth factor, also known as fibroblast growth factor-7 (KGF, FGF) and its receptor (KGFR). KGF exerts anti-apoptotic (by activating $\mathrm{Bcl}-2$ and $\mathrm{Bcl}_{\mathrm{XL}}$ ) and cell proliferation promotional activities (by activating the Akt or MAPK pathways) (325,326ab). While KGF induces differentiation into epithelial basal cells, it promotes AR expression (327). The LNCaP human prostate cancer cell line does not express KGF, but its exposure to external KGF promotes its growth; this is antagonized by flutamide or activin A or vitamin D3 analogs V and BXL-628, Université Libre de Bruxelles (328ab-330). AR is readily activated in prostate cancer cells by IGF, EGF, and KGF (331). Several human established prostate cell lines exhibited malignant growth without expressing KGF/KGFR (332), but high Gleason grade and metastatic tumor cells were KGF- and/ or KGFR-positive (333). Benign prostatic hyperplastic cells were negative, but $65 \%$ of human prostate cancer tumors were positive for KGF expression, especially in their hormone-insensitive stages (334). From these data, it appears that in benign prostatic hyperplasia, whether chronically infected or not, there is very little if any KGF activity, whereas the appearance of KGF activity signifies advanced and probably hormone-resistant disease. The rare paraneoplastic syndrome, bilateral palmar hyperkeratosis with prostate cancer (335) could be an adverse prognostic sign, especially if persists during therapy.

The WW (double tryptophane) domain contains oxidoreductase. The WWOX tumor suppressor gene is best understood in its relation to Tax oncoprotein of the human $\mathrm{T}$ cell leukemia virus, HTLV-I, and osteosarcomagenesis. While the HTLV-I Tax oncoprotein activates the NFkB pathways, WWOX inhibits the HTLV-I/Tax/NFkB pathway (336). In osteosarcomagenesis, WWOX is frequently absent (silenced; mutated). Active WWOX neutralizes the RUNX2 oncoprotein (runt box transcription factor) (337,338). Transduced adenoviral and lentiviral vectors are available for attempts at correcting WWOX deficiency. Tested in xenografted human breast cancer cells, WWOX was either re-instated through the adenoviral vector, or reactivated by the de-methylating agent 5 -aza-deoxycytidine. Active WWOX inhibited tumor cell growth (339). Naturaly, WWOX is activated by its ligand PPXY $(\mathrm{P}=$ proline, $\mathrm{Y}=$ tyrosine, $\mathrm{X}=$ any amino acid, $\mathrm{W}=$ tryptophane) (340). WWOX is suppressed in adenocarcinomas other than that of the breast (osteosarcoma, colon cancer, small cell carcinoma of the lung, prostate cancer) $(336,337,341-343)$. In small cell carcinoma, it is the BmI1 protein (vide supra) that suppresses WWOX (342). In prostate cancer, active WWOX not only suppressed tumor growth, but actually induced apoptotic deaths of tumor cells, but in actively growing tumors WWOX is silenced (344). Since WWOX suppresses HER $2 / \mathrm{neu}^{+}$breast cancer cells, it was tested against prostate cancer cells expressing ErbB2, equivalent of HER2/neu. The transcription factor Ap2y activates the Erb2/neu promoter. When ErbB2 is active in prostate cancer, it enhances AR expression and promotes hormone-independent growth (343). The tumor cells either function on minuscule 
amounts of testosterone, or activate their own testosterone production, the ligand for an autocrine loop (AR). The tumor cell carries out the conversion of adrenal androgens into testosterone and dihydrotestosterone and is able to synthesize androgens from cholesterol; these reactions are enhanced by insulin $(344,345)$. These androgens as ligands bind the AR in an autocrine circuitry. Cytochrome enzymes P (CYP) are active in this conversion. The CYP enzymes can be antagonized by abiraterone, but they can gain abiraterone-resistance (344).

The Sprouty 1 (Spry1) gene product protein is a tyrosine kinase receptor inhibitor for the fibroblast growth factor (keratinocyte growth factor, vide supra). Prostate cancer cells down-regulate Spryl both by hypermethylation of its DNA, or by silencing its mRNA by targeting it at its 3'UTR region with microRNA-21 (345). The high cysteine-rich protein gene (HPCX) encoded from locus Xq27-q28 is considered to be a susceptibility gene in hereditary prostate cancer. It is closely associated with genes SPANX (sperm protein associated with nucleus) and MAGE-C1 (melanoma antigen gene protein). The SPANX genes exhibit frequent gene deletions, duplications, homology-based sequence transfers and recombinations. The MAGE-C1 gene's mRNA offers 12 target sites to miRNAs. Gene MAGE-11 resides at Xq28; its protein product belongs to the 'cancer-testis antigen family'; it increases the transcriptional activity of the AR. AR is mapped to Xq11-12 (347-350a). The ectopically expressed super elongation complex gene SEC23A reduced the growth rate of human prostate cancer cell lines without inducing apoptosis. When its mRNA was targeted by microRNAs miR-200c and/or miR-375, this inhibitory effect was abolished (351). There appeared a report on genes in the prostate, which are activated by inflammatory events (301). Of the genes reviewed here, only SPANX appears to be labile and versatile enough to be up-regulated by an inflammatory process. The most recent assessment lists those genes that foretell aggressive disease, relapses and prostate cancer-related deaths. These genes are LEPR (the leptin receptor gene), CRY (the mammalian clock cryptochrome gene), RNASEL (the ribonuclease L gene; latent endoribonuclease, pro-apoptotic unless suffered gene polymorphism), the IL-4-gene, and the ARVCF (armadillo repeat protein deleted in velo-cardio-facial syndrome gene, $\delta$ catenin genes, neurojungin, plakophilin, plakoglobin genes) (352). Except for IL-4, the reactions of these genes to inflammatory signals is not well defined at all. The ARVCF gene family is best understood in the context of the metazoan evolution of the catenin gene family (353). The epigenomics of prostate cancerassociated genes (methylation and de-acetylation) (354), and the relationship of gene-derived mRNAs as targets for microRNAS reveals extraordinary complexity. MiRNA-141 levels correlated with those of PSA, lactic dehydrogenase, and circulating tumor cells during the clinical course of prostatic carcinoma (355). The miRNA-200c is activated by oxidative stress (in the inflammasome); by inhibiting oncogene $\mathrm{ZEB}$, it is more of a tumor suppressor gene (356); so it is, when it reduces adenocarcinoma formation in Barrett's esophagus (vide supra), or suppresses squamous carcinoma cells in the head and neck region $(85,357)$; but by reducing tumor suppressor Sec23A protein levels, miRNA-200c becomes a promoter of prostate cancer cell proliferation (350a). In comparison, miR-203 is expressed high in normal prostatic tissues, but it is down-regulated in cancerous tissues. In bone metastatic prostate cancer cell lines, miR-203 is active in inducing the reversal, the mesenchymal to epithelial reaction $(350 \mathrm{~b})$.

The Sox (vide supra) family genes are highly up-regulated in pluripotent embryonic stem cells to maintain clonogenicity, selfrenewal and pluripotency, therefore they are active in fetal life. Sox 9 is expressed in intestinal crypts and hair follicles (sites of high cellular activities). Stem cells in the basal epithelium of the healthy prostate express Sox9. The $\mathrm{sHH}$, Wnt/ $\beta$-catenin pathway regulates Sox9. Sox 9 is highly expressed in hormone-refractory prostate cancer cells. The Sox 9 mRNA can be neutralized by shRNA (358). The single exon sox 2 gene is embedded within a long non-coding RNA at 3q26.3-27, and exists also in an overlapping transcript: Sox2ot. The sox $2 o t \rightarrow$ SOX2OT is transcribed from a highly conserved element. The ncRNA exercises regulatory function over the expression of the sox 2 genes. In active sox 2 gene, lysine (K) 4 of histone 3 is trimethylated (H3K4me3); in inactive sox 2 gene lysine 27 of histone 3 is trimethylated (H3K27me3) (359).

In fetal life, Sox is an inducer of ectoderm and neurogenesis. It is down-regulated (silenced) upon differentiation. Sox2 undergoes amplifications and mutations, for example in squamous cell carcinomas of mucous membranes of the head and neck and esophagus (82). Up-regulated Sox 2 with or without Sox4, Nanog, Oc4, BMI-1, and Myc induces adenocarcinomas of breast, endometrium, and prostate (360-363). In the prostatic adenocarcinoma stem cells, Sox 2 with Nanog, Oct-4, Myc, Ras, Klf4 (Krüppel-like family transcription factor, from drosophila), Oskar (drosophila oocyte) co-exist and co-act. Sox is a major contributor to the grade of malignancy: with up-regulated CD44 and PSA; apoptosis resistance (without high Bcl-2, but with down-regulation of $\mathrm{Ca}^{2+}$ entry), high $\mathrm{Ki} 67$ ( $\mathrm{K}$ index nuclear protein for cell divisions), high Gleason score (8-9-10), and easy transplantability as xenografts. The Sox 2 protein was expressed both in the cytoplasm and in the nucleus of the cancer cells. These cancer cells proliferated at an accelerated rate. These cells resisted apoptosis-induction by thapsigargin and cisplatin. In some prostate cancer cells doxycycline induced shRNA release. In mice reeeiving doxycyclin in their drinking water, $\mathrm{SOX}^{+}$tumor xenograft grew very slowly (363).

Sox 2 being the major contributor to all these, when its mRNA is neutralized by a complementary short hairpin shRNA, there is a complete reversal of all malignant scores close to normal: drop of Sox protein, restored apoptosis susceptibility, decreased Ki67 due to a standstill of cell proliferation, drop of Gleason score, and loss of growth potential in xenografts (363).

Prostate cancer bone metastases appear 'blastic' (osteoblastic), but osteoclastic bone lytic activity is substantial. The runt-related transcription factor RUNX family proteins (RUNX2, pivotal in osteogenesis) in heterodimers with the Cbf- $\beta$ protein (C-repeat binding factor; core binding factor) are overexpressed in those prostate cancer cells that metastasized to the bone. These cells have already undergone EMT $(364,365)$. However, when an $\alpha_{\mathrm{v}}$-integrin antagonist re-arranged the tumor cell surface, E-cadherin expression went up and vimentin expression went down, forcing the cell to present itself with less mesenchymal and more epithelial phenotype, thus with reduced locomotion and metastatic spread (366). The RUNX complex continued to exert pro-metastatic acts. The DNA proviruses of 
mouse leukemia retroviruses readily integrated into the run $x 2$ locus (367). Regulatory Treg cells maintain Fox3 expression through the protective effect of RUNX/cbf complexes on the Fox3 mRNA (368). The RUNX/cbf complex suppresses the IL-4 silencer gene Il4, and thus releases IL-4 production (369). While RUNX maintains Treg and IL-4 levels, when anti-RUNX targeted therapy becomes available for the treatment of bone metastases by RUNX suppression, IL-4 levels and the supply of Treg cells may drop. Tumor cells notoriously utilize IL-4 (tolerance-induction) and Treg cells (immune T cell elimination), thus RUNX inhibition may result in some immunological benefits as well. The mcab denosumab is directed at the ligand of RANK, the activator receptor of $\mathrm{NF} \kappa \mathrm{B}$; it inhibits bone resorption by osteoclasts (370). The standard osteoclast inhibitors remain the zoledronic acid preparations.

The oncogenic DNA of high grade prostate cancer cells is endowed with a great deal of biological-hormonal, radio- and chemotherapy resistance. The Dendreon DC vaccine sipuleucel (Provenge), while immunizing against prostatic acid phosphatase, an antigen of minor significance; it barely prolongs life. The PROSTVAC-VF (Bavarian Nordic) contains a poxvirus (vaccinia and fowlpox virus) expressing PSA with immunostimulatory molecules with or without GM-CSF. The PSA-TRICOM induces powerful immune responses, but as yet without significant clinical benefits $(371,372)$.

Oncolytic viral therapy induces stabilization of disease, minor and partial responses (especially by intratumoral injection, that is, into the prostate) and only occasionally complete responses (usually of short duration). Clinically meaningful durable remissions would occur if antiviral immune reactions of the host could be suppressed, or by-passed by using serially and sequentially alternating different oncolytic viruses (164). Naturally oncolytic (Newcastle disease virus, attenuated poxviruses, reovirus), or genetically engineered oncolytic viruses (adenoviruses, herpesviruses, vesicular stomatitis virus, measles virus, etc.) are available in clinical trials (373-389).

Among others, the special AT-rich sequence-binding gene product proteins $($ Satb1, 2) regulate gene expressions in embryonic stem cells. Nanog is a major determinant of stem cell gene expressions. Nanog ${ }^{\text {low }}$ stem cells lose their pluripotency to differentiation into somatic cells. Nanog ${ }^{\text {high }}$ stem cells may self-renew, but remain undifferentiated. Low Satb1 protein levels favor the rise of Nanog ${ }^{\text {high }}$ stem cells. Satb2 acts by down-regulating Satb1. Both Satb1 and 2 proteins actually bind 5 '-flanking sequence of the Nanog gene. Further, in action, the Satb1 and 2 proteins form homo- and heterodimers. Thus, the balance of Satb1/Satb2 directs Nanog in the stem cell toward differentiation, versus the stem cell keeping its pluripoteny persistently (390). The persistence of pluripotency renders the stem cell conducive to malignant transformation. The transcription factor FoxP3 suppresses the Satb gene in breast and prostate cancer cells; its effect is regulated by miR-7 and miR-155 (391). This system is deleted in cancer cells, and is awaiting for its re-insertion by a viral vector. However, the Satb1 mRNAs can be destroyed by a small hairpin RNA. The uniquely prostate cancer specific gene's DD3 (differential display code 3) presence in prostate cancer (DDR3/PCA3) could regulate as a promoter the expression of the shRNA $(392,393)$. An oncolytic adenovirus was constructed to express the gene of SATB1-shRNA under the DD3 promoter regulation. This adenoviral vector expressed the anti-Satb1 shRNA in prostate cancer cells (cultured in vitro) and was cytopathic to the tumor cells (394).

The genomic complexity of prostate cancers is extraordinary. While seven genetic patterns have been fairly well sequenced (395), many more remain still unrecognized. In addition to the well known androgenic pathway, prostate cancer cells overexpress EGF-R, IGF-R and prolactin receptors (396-399). The appearance of periostin (and tenascin) exclusively in the tumor stroma indicates that they are tumor-induced and as such they should be targeted for therapy (400).

However, this tumor is not targeted as yet with individualized gene therapy. The primary tumor is treated with the 'fits all' radical prostatectomy, or high dose linear accelerator external beam, and brachy-radiotherapy with or without androgendeprivation therapy. The 'Beaumont regimen' revealed that in high risk disease (Gleason $>8$ ) apparently still confined to the gland (always questionable), hormonal therapy (non-curative, palliative) delaying high dose radiotherapy was of disadvantage ('detrimental') (401-403). This is known since 2007, yet androgen-deprivation is still recommended to precede radiotherapy. Unless leuprolide and/or bicalutamide induce tumor cell apoptotic deaths (other than promoting the 'metabolic syndrome' of the patient), the tumor cells arrested in $\mathrm{G}_{0}-\mathrm{G}_{1}$ survive the radiotherapy (402). These are the metabolically active, but rendered irreversibly stagnant (not replicating), 'senescent' tumor cells. This is the situation in which AR-negative neuroectodermal prostate cancer cells may arise (without producing PSA).

The best results for high risk disease would appear to be the immediate high dose interstitial radiotherapy (iridium Ir-192 source brachytherapy within the gland and its immediate periphery), preceding the external beam intensity-modulated radiotherapy (IMRT). External beam radiotherapy may induce late carcinogenesis (adenocarcinomas, sarcomas) in the pelvis, and brachytherapy in the prostatic urethra $(404,405)$. These tumors probably arise from radiation-induced gene-mutated cells escaping apoptotic death, but without inflammatory etiology. The predicted incidence of radiotherapy-induced tumors is as high as $1 / 250$ at 5 years, and 1/70 at 10 years (405).

For metastatic disease, no curative treatment is available, but various cytotoxic chemo-, or targeted therapeutic regimens with alternating androgen suppression prolong life. Epigenetic interventions (cancer suppressor gene demethylations or histone re-acetylations) hold promise bordering the spectacular, for the restoration of cancer suppressor genes and for the suppression of oncogenes $(354,406)$. For the promise of targeted therapy, abiraterone inhibiting the cytochrome enzyme CYP17A1, and the $s r c$ family kinase-inhibitor, dasatinib, are the examples (407-409). Dasatinib is a wide-spectrum oncogenic kinase inhibitor, acting against the break-point/Abelson fusion oncoprotein (BCR/ABL), platelet-derived growth factor receptor (PDGF-R) and c-KIT (kinase target). However, the sequentially and serially mutated oncogenic DNA switches from those suppressed to several new (more than 3) oncoprotein-driven pathways.

For the promise of improved immunotherapy, ipilimumab releases autoimmune reactions against cellular targets masquerading as 'self' and as such, it could induce durable remissions in cases of prostatic carcinoma $(407,409)$. Subclasses of cytotoxic/cytolytic lymphocytes are able to recognize and attack transforming stem cells. In order to attack prostate stem cell 
antigen-expressing senescent prostate cancer cells, the $\mathrm{T}$ cell receptor could be re-directed by bispecific antibodies $(410,411)$.

Estimations in the literature put the incidence of inflammation induced cancers in general at the $35-40 \%$ range, except for prostatic carcinoma: there $85 \%$ for inducing inflammatory somatic mutations, and $15 \%$ for various forms of inherited (SNP, gene mutations and familial vertical transfers) etiology. Non-steroidal anti-inflammatory agents and anti-oxidants [aspirin, ibuprofen, celecoxib, curcumin (Anand P, et al, Press release from M.D. Anderson Hospital, Houston, TX, Sept 8, 2011), resveratrol, epigallocatecholamine-gallate, licorice roots' triterpenoids' metabolite glycyrrhizin, lycopene, quercetin, pomegranate polyphenols, silibinin, soy isoflavons, vitamin D analogues, etc.] are of some preventive value in reducing incidence, but have no proven clinical efficacy against established disease; however, it is claimed that they improve the efficacy of standard therapy, and in combinations (the anti-inflammatory celecoxib and antiandrogen hormonal therapy) may induce remissions (412). The preventive value of dutasteride is in doubt: indeed it has utterly failed to prevent high grade disease. These data explain the increased attention inflammation-induced prostate cancer, frequent, most versatile, and devastating, received in this article.

Endometrial adenocarcinoma. The menstrual cycles were recognized to be inflammatory processes imitating wound healing and inviting the outpour of inflammatory cells (leukocytes, macrophages, NK cells). Even in the menopausal stage, the endometrium is receiving an influx of estrogens without the counterbalance of progesterone; androgen levels may rise. Carcinogenesis in the endometrium may occur both by the intrinsic (cancer is initiated first and then is supported by inflammatory cytokines), or by the extrinsic (chronic or periodical inflammatory processes induce carcinogenesis) pathways. Inactivation of PTEN and consequential activation of the PI3K, and Akt 'cell survival' pathways follows. Cytoplasmic NFKB is liberated to transfer into the nucleus, where it activates a series of inflammatory genes. Chemokine and cytokine showers imbue the tissues. The first gene to undergo point mutation is the Kras (Werner Kirsten's Moloney virus-induced rat sarcoma). The transforming tissues exude prostaglandins and cyclooxygenases. Irreversible malignant proliferation of the endometrium takes place $(413,414)$.

The Sox 4 oncogene at $6 \mathrm{p} 22$ encodes the Wnt/sHH oncogene cascade with Notch and TGF $\beta$ signaling. Sox 4 is under the suppressive control of the non-coding small miRNA-129-2. In cancerous endometrium the $\mathrm{CpG}$ islands of miRNA-129-2 are hypermethylated and thus silenced. In response, the Sox4 oncoprotein is up-regulated. Patients with hypermethylated miRNA-129-2 experience poor prognosis and short life expectancy. DNA demethylation (for example, with 2'-deoxy5-azacytidine) and histone re-acetylation (for example, with the deacetylase inhibitor trichostatin) with resulting restoration of miRNA-129-2 activity promptly stabilize the course of their disease. Sox 4 oncoprotein levels drop; cancer cell proliferation falls to a standstill. Patients with stable disease or actual remission experience prolonged survival $(361,362)$.

Melanoma. Ultraviolet light- (UV irradiation-) induced acquired somatic mutations against the background of UV light-induced subcutaneous inflammation induce this tumor. The inflammatory mechanisms, if any, that induce visceral, or uveal, melanomas, are unknown. Oncogene B-raf (rat fibrosarcoma) is the most common mutation encoding a serine-threonine oncoprotein kinase. This oncoprotein kinase induces abnormalities of the mitotic spindles with aneuploidy in the melanocytes (415). This author repeatedly reviewed his experience with this tumor, both at the basic science level and in the practice of medical oncology $(416,417)$. Patients with melanoma express weak tumor-specific immune reactions in addition to the 'non-specific inflammatory background'. In past decades, Bacille Calmette-Guerin (BCG) was widely used to stimulate the anti-melanoma-specifc immune reactions (418). Some human melanoma cells activate an endogenous retrovirus, whose role in the etiology of the disease, and in the induction, or suppression, of host immunity remain unresolved (419-421).

As to pre-molecular medicine era immunotherapy, Newcastle disease virus-, or influenza A virus-oncolysates cancelled or retarded the appearance of metastases after surgical resections of metastatic lymph nodes in stage III disease; in stage IV disease significant prolongation of life was observed in not prospectively randomized patients treated with viral oncolysates $(164,422,423)$. However, overvaccination could be detrimental. Was this due to chronic inflammatory reactions, or overstimulation of leukocytes and macrophages by GM-CSF? Eggermont analyzed the problem in his superb editorial (424). Patients with metastatic melanoma eliminate the Th1-type internal immunological environment and replace it with the actively tumor-tolerant Th2-type setting (425). Through the expression of the ICOS-L/B7H ligand (inducible $\mathrm{T}$ cell costimulatory ligand) by melanoma cells, the host generates large tumorprotective Treg cell populations (426).

The broken chromosomes 1 and 10 undergo mismatched fusion encoding the Fas receptor from 10q23-26 on the cell surface, while the cytoplasmic tail is the granulocyte colony stimulating factor encoded from 1p32-34. Capturing the Fas ligand (FasL $\rightarrow$ FasR) induces mitoses of these melanoma cells (Horvath JC, et al, Proc 89th Annual Meeting American Association Cancer Research, New Orleans, LA, 39: 584, abs. 3971, 1998) (427). Glioblastoma cells utilize the FasL $\rightarrow$ FasR pathway for their divisions (vide supra) (154). The malignant cell DNA can use chromosomal breaks and fusions to its own advantage. The malignant cell DNA enlists microRNAs for its propulsion; in melanoma, the miRs-30b/30d silence the intercellular communication enzyme $\mathrm{N}$-acetyl- $\alpha$-D-galactosamine polypeptide or $\mathrm{N}$-acetyl galactosamine transferase (GALNT7) and induce the lymphokine IL-10 for promoting tumor cell spread in an immunologically tolerant microenvironment (428).

The mcab ipilimumab and tremelimumab, and the mutated B-RAF oncoprotein inhibitor vemurafenib lead the proceedings toward the possible cure of metastatic melanoma.

Sarcoma. The classical tumor with possible inflammatory etiology is the malignant fibrous histiocytoma (429-431). Under the inquiry 'malignant fibrous histiocytoma' PubMed lists several examples of this tumor occurring in a lumbar abscess, in the retroperitoneum, in the thymus, in the kidney, in the spermatic cord, etc. HIF promotes the inflammatory process. In the chronic inflammasome, reactive oxygen and nitrogen 
(N-synthase, iNOS) species induce DNA strand mutagenesis. A mutagenic product is 8-nitroguanine. Co-expression of 8 -nitroguanine in the nuclei and HIFl $\alpha$ in the cytoplasm of the targeted cell resulted in $\mathrm{NF} \kappa \mathrm{B}$-driven sarcomagenesis with adverse prognosis (432).

Inflammatory lymphocytes reactive to sarcoma cells usually represent complex clones of lymphoid elements. Autologous small round immune $\mathrm{T}$ cells and autologous and allogeneic large granular lymphocytes (recognized as NK cells) react to sarcoma cells exploring the target in emperipolesis and either practicing tolerance, or launching a cytolytic attack. Cytotoxicity is expressed either (or both) as cytoplasmic lysis, or 'nuclear clumping', a morphological sign of apoptosis (433). In a desmoplastic small tumor cell sarcoma, talcum-induced reactive macrophages attacked tumor cells in the pleural cavity (430).

An established human chondrosarcoma cell line was observed to undergo a slow process of differentiation in vitro. The chondrosarcoma cell line was in advanced passages (over 30 transfers in the 5th year of its establishment), thus considered to be free of any healthy stromal cells, including fibroblasts. To the 33rd passage population of the tumor cell line, small compact, and large granular buffy coat-derived lymphocytes ( $\mathrm{T}$ cells and NK cells) of a healthy donor (JGS) were added. The large often polyploid tumor cells were explored by the donor lymphocytes (in emperipolesis), but suffered very rare incidence of cytolysis or 'nuclear clumping'. Dividing tumor cells gradually yielded elongated fibroblast-like cells in their progeny with single round nuclei without aneuploidy. These cells eventually showed signs of senescence (documented in serial microphotography). The laboratory was experienced in punctually marking and observing cultures with a very good record of avoidance of mix-ups or cross-contaminations $(321,434)$. The unexplained (and held doubtful) 'spontaneous' differentiation of a chondrosarcoma cell line can now be explained by the reaction of PPAR $\gamma$ uniting with their ligands (435). The artificial ligands of the system (the glitazones, indomethacin) are known, but if lymphocytes could deliver the natural ligands, that has not as yet been explored. If found, a balance of sarcomagenesis in the inflammasomes could be envisioned: the activation of oncogenes counterbalanced by re-differentiation of the transforming cells through ligandactivation of their PPAR $\gamma$ receptors. However, the PPAR $\delta$ pathway (not to be mistaken for the PARP pathway) appeared to be tumor-promoting in adenocarcinomas (vide supra). A chain of events were recognized following the growth arrest of chondrosarcoma chondrocytes by FGF beginning with the dephosphorylation of the RB protein $\mathrm{p} 107$, and repression of the E2F genes (436).

Retrovirally induced sarcomas dominate in the animal world from fish up to simian hosts (429), but human sarcomas appear as an outstanding exception. Even when retroviral particles are visualized in a human sarcoma, these have not as yet been isolated and identified (431). It is not clear at all how human sarcomas may be induced by inflammatory reactions, and how inflammatory cells (cytolytic lymphocytes) are actually mobilized to attack them (433). One can envision inflammasomes generating sarcomas. However even the most scholarly reviews of inflammasomes abstain from such suggestion (437). Sarcomagenesis in the inflammasome: is it overlooked or is it not happening?
Instead of the expected sarcomagenic retroviruses, two human sarcomas harbor herpesviruses: in multifocal leiomyosarcoma of children EBV, and in all forms of Kaposi's sarcoma, the human herpesvirus-8 (HHV-8, KSHV) prevail. However, both herpesviruses may activate, or co-exist with, endogenous retroviruses $(108,433)$. Herpesvirally induced sarcomas (Kaposi's sarcoma; EBV-induced leiomyosarcoma), evoke their own virus-specific immune reactions (vide infra). Weather these immune reactions are defensive against, or promotional to, these tumors, will be best settled, if both directions are recognized to co-exist (vide infra).

Concluding remarks. It was in 1972, when Ruckdeschel et al described their clinical observation on postoperative empyemas reducing relapses of surgically removed lung cancers (438a). A decade later, postoperative intrapleural administration of BCG not only failed to inhibit, but it possibly enhanced, recurrent tumor growths of lung cancer (438b). Nowadays, inflammation is named in the pathogenesis of lung cancer $(439,440)$ and we construct immunotherapy for lung cancer 'from inflammation to vaccination' (441).

In the 1970's, the re-organized and lavishly supported NIH/ NCI for the Conquest of Cancer issued contracts and grants for the 'non-specific immunostimulation of patients with leukemias and solid tumor cancers'. It would be interesting to find out if corynebacteria (C. parvum) actually promoted tumor growth. BCG did better: it induced partial regression of some tumors (melanoma, transitional cell carcinoma of the urinary bladder). It became a vogue and a bandwagon to show 5-10\% improvements of chemotherapy results, when BCG was added in the case of practically every tumor; it was then 'the state of art' therapeutical intervention. Yet it has been almost completely abandoned. There was a balance to be found between oncogenic and oncosuppressive immune reactions. The oncogenic inflammatory reactions were not immediately recognized or simply overlooked, and the oncosuppressive immune reactions were overestimated.

At that time (the 1960's), the NIH/NCI steadfastly withheld funding for a 'tumor-specific' immunotherapy protocol (viral oncolysates against melanoma and sarcoma) with or without chemotherapy. Grant applications for viral oncolysate vaccine therapy with chemotherapy combination were rejected as 'mutually exclusive and useless'. However, in the last decade over a dozen clinical trials prove that tumor-specific immunotherapy is additive or even synergistic with chemotheapy, as if chemotherapy inhibited the tumor-promoting inflammatory reactions, and opened up an avenue for the immunotherapy to target the tumor: 'Evidence accumulating in support of cancer vaccines combined with chemotherapy' (442).

Considering the impressive documentation of inflammatory carcinogenesis, the induction of any new inflammatory reaction (oncolytic viruses, vaccines) into a tumor-bearing host should be carefully balanced. Oncolytic viruses and vaccines may work best when combined with a moderate chemotherapy regimen (reducing the tumor load and controlling the oncogenic inflammatory reactions). The newly induced inflammatory reaction of a vaccine or an oncolytic virus should be very specific and forcefully directed against its target; it should be re-inforced by chemo-cytokines and microRNAs with the proven reputation to be pro-apoptotic and anti-carcinogenic. 


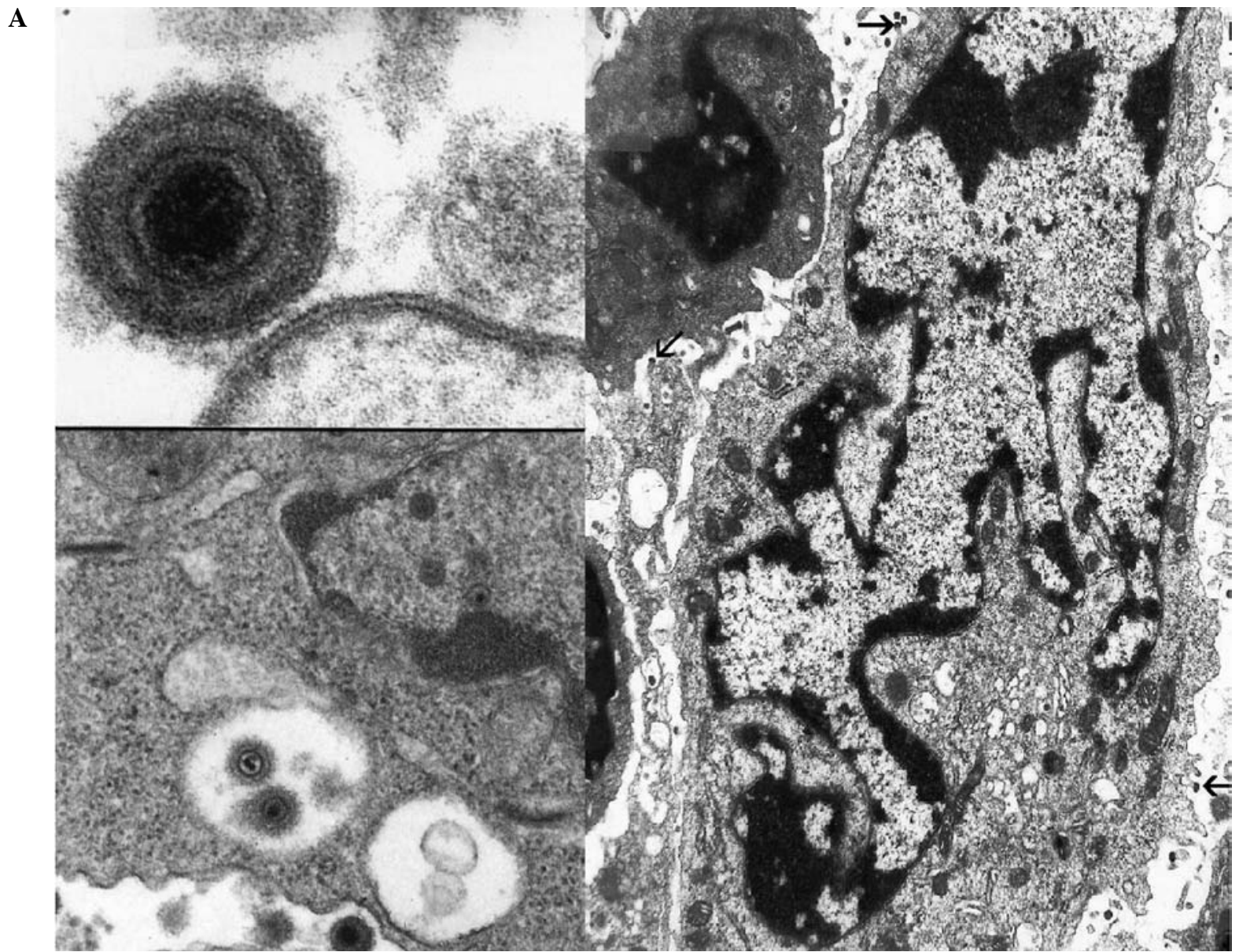

Figure 4. (A), Kaposi's sarcoma cell from the pre-AIDS era shows immature intranuclear, and mature cytoplasmic herpesviral particles (not known then to be HHV-8). Original magnification, x47,500. (B), Budding retroviral particles (not HIV-1) from Kaposi's sarcoma cell of the same patient. The activated endogenous retroviral particles by histochemical stains and attempts at culturing them were not identical with HTLV-I. Original magnification, x16,000. In the case of diminutive reproduction, please use magnifying lens to view the virus particles. Transmission Philips electron microscopy from the material of the Veterans' Medical Center, Department of Pathology (Head, Professor Ferenc Györkey), Houston, TX, USA.

\section{Pathogens with potentially oncogenic genomic sequences}

Viral I. Kaposi's sarcoma cell nuclei are loaded with immature HHV-8 particles and frequently release an endogenous retrovirus (similar to, but not identical with, human $\mathrm{T}$ cell leukemia virus, HTLV-I) (Fig. 4: if printed in a diminutive size, please view under a magnifying glass).

KS-associated herpesvirus (KSHV, HHV-8) reprograms vascular endothelial cells into lymphoendothelial cells (KS cells). The virus infects B lymphocytes and turns them into plasmablasts. Occasionally tonsillary $\mathrm{T}$ lymphocytes are also infected. KS cells express the c-Kit (kinase tyrosin) receptor. The major viral oncoprotein is the $\mathrm{G}$ protein-coupled receptor (guanyl nucleotide-dependent protein). Open reading frame K12 encodes kaposin A accelerating the MAPK and PI3K pathways, and kaposin B producing endothelial cell regulator Prox 1 (after drosophila gene prospero) (443). The KS cell genome induces anti-apoptotic Bcl-2, down-regulates $\mathrm{Rb}$ and p53, induces mTOR and Notch proto-oncogenes, and releases autocrine, or receives paracrine growth factors: IFN $\gamma, \mathrm{TNF} \alpha$, IL-6 (reviewed in ref. 444). KSHV induces ubiquitin ligases and HIF-1 $\alpha$, thus contributing to the Warburg effect of anaerobiosis (445a). The viral FLICE inhibitory protein FLIP (FADD-like IL-1 $\beta$ converting enzyme, Fas death domain) contributes STAT activation and spindle cell formation. Viral ORF57 encodes vIL-6; its mRNA transcript accumulation (TA) and miRNA recognition elements (MRE) stabilize viral and host cell IL-6 mRNAs $(443,445 b)$.

The human interferon (IFN) regulatory factor-related KSHV gene (vIRF-3) inhibits types I and II IFN production in effusion lymphoma cells; it inhibits histocompatibility complex class II expression (thus antigenic presentations). In a peculiar way, IFN $\gamma$ production was also inhibited (whereas IFN $\gamma$ was shown to be a growth factor for KSHV) $(446,447)$.

KS cells release 17 adenylated miRNAs. KS miR-K12-7 and miR-K12-7-5p target the 3'UTR of RTA (replication and transcription activator) and the viral immediate early gene, thus maintaining viral latency (448). The genome of KSHV releases 12 microRNAs (miR) from its LANA-associated intron. These miRs post-transcriptionally regulate host cell gene expressions by silencing the corresponding mRNAs. The translation of the miR of thrombospondin is inhibited; thrombospondin is a strong tumor suppressor and anti-neoangiogenic factor (449). The nuclear ribonuclease Drosha-cleaved hairpin RNA exits the nucleus with exportin5, is processed by cytoplasmic ribonuclease Dicer, is incorporated into the RNA-induced silencing complex and thus is guided to the 3'UTR of the targeted mRNA, which is degraded. These viral 
miRs have no sequence conservation to metazoan miRs. But the KSHV-induced miR-K12-11 is homologous to the host lymphoma cell-induced miR-155. These miRs regulate the BTB (bric á brac) and CNC (cap'n collar) homology domains, abbreviated as BACH (450-453). BACH-1 is a negative regulator of transcription recognizing antioxidant response elements within gene promoters. In suppressing BACH-1, the membrane-bound subunit of the amino acid transporter xCT increases, thus intracellular glutathione stores remain full providing protection against oxidative stress. Further, xCT is the cell surface receptor for the entry of KSHV (454). The Orf63 of KSHV is homologous to the cellular NLR (nucleotide binding and oligomerization, leucine-rich repeat), which activates innate immune reactions in the inflammasome. KSHV Orf63 protein blocks the cellular NLR at its initiation (IL-1 $\beta$, caspase-1 and IL-18), thus the virus escapes the activation of innate immunity and its consequences (455).

Classical pre-AIDS KS had the reputation to be a tumor afflicting immunosuppressed hosts (immunosuppressed organ transplant recipients). The classical Southern European patients were elderly. Even in the virulent African KS, an immune system exhausted by bacterial, protozoal and helminthic infections was blamed for the acceptance of these tumors. Severe immunosuppression in HIV-1-infected patients with the acquired immunodeficiency syndrome was thought to render these patients defenseless to these tumors. However, in the most paradoxical way, the recrudescence of virulent $\mathrm{KS}$ in these patients after viral inhibitory therapy-induced remission of AIDS with recovery of the immune competence proved that KS could be subject of inflammatory oncogenesis. A long list of references attest to this concept (E.A. Mesri citing articles from Y. Aoki, C. Boshoff, J.L. Douglas, B. Ensoli, R.C. Gallo, P. Monini, L. Pantanowitz, G. Riva, etc.) (456). To the dissatisfaction of this author, these articles speak in generalities and fail to pinpoint the exact tumor-supporting infectious-immunological mechanisms. Constituents of the inflammatory infiltrates around the tumors are listed one by one (DCs, several different classes of lymphoid cells including plasma cells, NK cells, monocytes/ macrophages), but without their roles in oncogenesis, or in the defense against it, specified. In patients with KS, immune T cells and cytolytic NK cell are generated, exert cytotoxity to malignantly transformed cells, but eventually fail to control tumor growth (457-459). However, there is recovery from KS, that is regressions of these tumor may occur, for example in organ transplant recipients, when the immunosuppressive medication is withdrawn or modified (460-462). Immunosuppression with rapamycin exerts also anti-tumor effects by the inhibition of the mTOR pathway (mammalian target of rapamycin), thus it may reduce post-transplant Kaposi's sarcoma generation with retention of the graft. HHV-8/KSHV remains in latency failing to enter its lytic cycle in rapamycin-treated virus-carrier cells. Rapamycin specifically inhibits the replication and transcription activator (RTA), the lytic switch protein (463).

Viral II. Dreyfus and Sinkovics independently have made the elaborate suggestion, that an ancestor of the gammaherpesviruses transferred the genomics (genes, operons) into the ancestors of sharks, which in unison for the first time, encoded the adaptive immune system (reviewed in ref. 62). The HHV-4 Epstein-Barr human gammaherpesvirus (EBV) causes infec- tious mononucleosis; while the infected large B lymphoblasts are rejected by immune $\mathrm{T}$ cells, epigenomic latent viral genomes remain silent for the rest of the patient's life. The viral genome persists epigenetically located in memory B cells. EBV contributes to the pathogenesis of lymphoepithelioma-nasopharyngeal carcinoma (type II latency), African Burkitt's lymphoma (type I latency with EBNA-1 expression), NKT cell lethal midline granuloma (type II latency), and Reed-Sternberg cells of Hodgkin's disease (type II latency with EBNA-1 and LMPs expression). EBV immortalizes human B lymphocytes in in vitro suspension cultures (type III latency with expression of EBNA-1-6 and LMP1, 2A, 2B); it induces posttransplant lymphoproliferative disease; it induces the $\mathrm{EBV}^{+}$diffuse large $\mathrm{B}$ cell lymphoma in the elderly; it is associated with brain lymphomas in patients with AIDS, it is present in multifocal pediatric leiomyosarcomas, and in gastric carcinomas in Japan (reviewed in refs. 62,443). In autoimmune diseases (lupus erythematosus, multiple sclerosis), there is an interaction between EBV and latent endogenous retrovirus(es). Herpesviruses trigger the maturation of latent endogenous retroviruses, which appear budding from lymphoid cells, Reed-Sternberg cells, and even the transformed lymphoendothelial cells of Kaposi's sarcoma $(62,108,178,464)$.

The EBV genome is present in $\mathrm{T}$ cells of angiocentric lymphomas presenting as 'lethal midline granuloma' (465), a pathological entity different from the lethal midline granulomas caused by malignantly transformed NKT cells (466-468). In patients with X-linked lymphoproliferative disease with SAP gene mutation (SLAM-associated adaptor protein containing SH2 domain; SH2, Src homology; SLAM, signaling lymphocyte activation molecule), EBV infections are life-threatening (469). It is the tolerogenic, Th2-type immunological environmentinducer IL-10 that activates the expression of EBV's LMP1 in Burkitt's lymphoma and in NK cell lymphomas (470). After EBV infection in healthy individuals, the viral genome rests in a circular configuration in the epigenome. The cytokines IL-4, IL-13 and IL-21, DNA-methylations and histone acetylationsdeacetylations control the mode of expression of the latent EBV genome. For the host's health, the most ominous change is the expression of the latent membrane protein (LMP1, 2A, 2B) (471-475).

In order to neutralize host cell mRNAs, which are to be translated into defensive inflammatory proteins, the EBV genome generates numerous (may be over 25) microRNAs (EBERs) $(474,475)$. The EBV genome BamHI-A rightward fragment (BARF) and BamHI (Bacillus amyloliquefaciens endonuclease) fragment $\mathrm{H}$ rightward open reading frame (BHRF1) release the viral microRNAs most frequently in the case of type III latency in nasopharyngeal tissues. EBV miRNAs target chemokine CXCl-11, inhibit transition from latent to lytic viral replication, and suppress the p53 up-regulated mediator of apoptosis (PUMA), and regulate the expression of LMP (476). The N-terminus of the BARF protein activates Bcl-2 and proto-oncogene c-myc. Soluble BARF protein imitates a CSF and thus is mitogenic; it suppresses IFN $\alpha$ production. Gammaherpesviral miRs are orthologs and interrelated (shared seed sequences) among Marek's disease HV of turkeys, KSHV and others (miR-155) (477).

IL-4 and IL-13-induced STAT pathway elicits EBV's LMP induction (471-473). LMP is a proto-oncogene. EBNA-2 and 
EBNA-5, IL-10 and IL-21 induce LMP expression. The truncated LMP transforms and immortalizes B cells and activates Bcl-2, NFkB, STAT-1 and JAK3 (janus kinase). LMP suppresses pro-apoptotic p53 and Bax signals (Bcl-2-associated $\mathrm{X}$ protein). LMP induces miR-29b, and thus down-regulates TCL1 ( $\mathrm{T}$ cell leukemia) oncogene (478). The negative regulation of an oncoprotein by EBV LMP must have some so far unclear biological implications. LMP2A activates PI3K/Akt/mTOR pathways. In a mouse model of Burkitt's lymphoma with c-myc translocation, rapamycin suppressed splenomegaly and tumor metastases (479). Host snail and twist gene activations lead to epithelial-tomesenchymal transition of EBV-infected cells in NP carcinoma. Translocated $\beta$-catenin from cytoplasm to nucleus activates proto-oncogenes (480). LMP1 increases intranuclear transfer and accumulation of $\beta$-catenin (481). The LMP of EBV activates STAT protein kinase C (PKC) and ERK (extracellular signal regulator kinase) (482). Malignantly transformed cells operate these very same signaling pathways.

In the EBV granuloma/inflammasome there are lymphoid cells infiltrating $\left(\mathrm{CD} 8^{+} \mathrm{CD} 4^{+} \mathrm{T}\right.$ cells and $\mathrm{CD} 4^{+} \mathrm{CD} 25^{+} \mathrm{Fox} 3^{+}$Treg cells, NK cells, B lineage cells depositing immunoglobulins), mast cells, neutrophil and eosinophil leukocytes and inflammatory lymphokines (IL-1 $\beta$, IL-3, IL-6, IL-8) and chemokines (CXCL1, the IL-8 analog, CXCL22, CXCL5-6), further, RANTES and MCP1 (regulated on activation normal T cell expressed secreted; macrophage chemoattractant protein) and $\mathrm{NF} \kappa \mathrm{B}$ and $\mathrm{TNF} \alpha$ lubricate the interstitium. These agents one by one or in unison maintain an inflammatory environment serving the virus and the tumor to the disadvantage of the host (483).

EBV-associated multifocal smooth muscle tumors occur worldwide and target children. These tumors are now being recognized in Singapore as leiomyosarcomas in $\mathrm{EBER}^{+}$ immunosuppressed kidney transplant recipient adults. EBV latency was considered to be type II (vide supra). The tumors persisted and advanced after the discontinuation of the immunosuppressive medication (cyclosporine and prednisone). The tumors activate the mTOR/Akt pathway of oncogenesis. The Ras association domain family gene (RRASSF1A) was methylated (considered to be silenced) and the MGMT gene (methylguanine-DNA methyltransferase) was hypomethylated (yet considered to be silenced) in every case. It is known that EBV LMP attacks (suppresses) RASSF1A. The mTOR inhibitor sirolimus (rapamycin) induced remission in a patient with a leiomyosarcoma in the liver. The tumors were chemotherapy-resistant. Demethylation of the RASSF1A gene could be attempted, however, demethylators can liberate the latent EBV genome (484). A lung transplant patient developed $\mathrm{EBV}^{+}$ uterine leiomyosarcoma (485). A retroperitoneal inflammatory leiomyosarcoma was first mistaken for a malignant fibrous histiocytoma (vide supra) (486). It is not known if this tumor was EBV-associated. May be all leiomyosarcomas should be submitted to the Chang-Moore digital transcriptome subtraction technology in search for an epigenetically hidden circular EBV genome (as this technology worked for the recognition of HHV8/KSHV, vide supra).

In activating the ras (Harvey or Kirsten rat sarcoma) proto-oncogenes gammaherpesviruses can induce a cascade of intracellular events, the most prominent being the pre-oncogenic PIK3 ‘cell survival pathway' (487a).
Viral III. In the chronically inflamed uterine cervix, the high risk papilloma virus deposits its E6 (to target p53) and E7 (to target RB) oncogenes; after neutralizing these tumor suppressor genes, actual activation of oncogenes follows (487b). High risk human papillomavirus, (HPV-16/18), causes squamous cell carcinomas of the uterine cervix, and with or without $\mathrm{EBV}$, is responsible for carcinogenesis in the oral cavity and nasopharynx $(487 \mathrm{c}, 488)$. These are the lymphoepithelioma-like HPV-related head and neck carcinomas $(489,490)$. In North America the incidence of $\mathrm{HPV}-16^{+} \mathrm{EBV}^{-}$nasopharyngeal carcinomas are increasing (491). High risk HPV-16 shows up in anal warts (491) and in anal squamous cell carcinomas (493).

The target cells of the virus are keratinocytes and other epithelial cells. Against the background of non-specific chronic inflammation (in the uterine cervix, in the nasopharynx, in the ano-rectum), the HPV-16 E6 and E7 oncoproteins heterodimerize with the p53 protein and the RB protein and these complexes are removed by ubiquitination. HPV-16's oncogenes E6 and E7 share a common promoter. The complex formation involves the LXCXE (L, leucine; C, cysteine; E, glutamine and $\mathrm{X}$, any amino acid) motif of E7 fitting into a pocket of the RB protein (494-496). The virally infected cell loses its major propensity toward apoptosis and cell cycle control. Degradation of the regulator of hTERT, nuclear factor binding box 1 (NFX1), removes hTERT repression (human telomerase reverse transcriptase) and thus immortalizes the infected cell. Normally NFX1 promotes the generation of protein p105, an inhibitor of NFкB (497). With degraded NFX1, the p105 protein is not generated; consequentially NFאB levels rise. The virus induces centriole overduplication and the infected cells divide with supernumerary mitotic spindles; consequentially, aneuploidy sets in and the chromosomes become missegregated. HPV-16 activates the polo-like kinase 4 promoter; plk4 mRNAs are overproduced and the cell cycle accelerates its G2 to M progression. Under hypoxic inflammatory conditions, E6 inactivates the human tumor suppressor de-ubiquitinase gene CYLD (cylindromatosis); this act rescues NFאB for unrestricted activity (498-500a). The inflammasome of the uterine cervix is bathed in high levels of the tolerogenic IL-10. It is the peripheral blood mononuclear cells that produce IL-10; the CpG islands of the proximal promoters for IL-10 production in the epithelial cells were silenced by hypermethylation (500b).

There appears to be an abundance of identified and unidentified microRNAs released from the genomes of papillomavirus-infected tumor cells and from the viral genome (501). An outstanding example is miR-200a, which down-regulates oncogenes ZEB1, 2 and TGF $\beta$ (vide supra). These oncogenes work through the E-cadherin/ $\beta$-catenin pathway in promoting EMT of the tumor cells, thus initiating metastatic spread. Expression of miR-200a suppresses tumor metastases (502).

The oncoprotein E7 down-regulates the oncosuppressor and cell differentiation inducer miR-203. The target for negative regulation by miR-203 is p63, the level of which gradually drops as stem cells differentiate. Viral oncoprotein E5 lowers miR-203, and raises p63 levels. The cancer cell proliferation inducer miR-146a is active in cervical cancer cells (also active in breast, pancreatic and some prostate cancer cells, as well as in psoriasis). The immunosuppressive miR-146a is strongly up-regulated in cervical carcinoma cells; miR-146a 
is up-regulated by oncoprotein E5. From the point of view of inflammatory carcinogenesis, it is important to observe the suppressor of cytokine signaling proteins, SOCS-3: they suppress IL-6 and IFN production. Whereas, miR-203 targets SOCS-3 for negative regulation, thus inducing increased inflammatory responses (503).

Host immunity can eliminate early lesions of cervical carcinogenesis, but the immunity thus gained may be ineffective in protecting against a newly acquired papillomavirus infection (504). High grade cervical dysplasia persists with the continuous expression of the HPV-16 E7 oncoprotein. This oncoprotein overrides the suppressor activity of $\mathrm{p} 21^{\mathrm{cip} 1}(505)$. Tumor-infiltrating macrophages are subverted and assume the CD45 ${ }^{+}, \mathrm{F} 4 / 80^{+}$ and $\mathrm{CD} 11 \mathrm{~b}^{+} \mathrm{M} 2$ phenotype of tumor-associated macrophages (TAM). TAM expressed IL-10 and Fox 3 and thus antagonized CD8 tumor-immune T cells. $\mathrm{CD} 45^{+} \mathrm{CD} 11 \mathrm{~b}^{+} \mathrm{Grl}{ }^{+}$myeloid-derived suppressor cells also invaded the tumors. Depletion of TAM with clodronate liposomes restored tumor-immune $\mathrm{T}$ cell activity with tumor regression (506). Mice carriers of lung-metastasizing $\mathrm{E}^{+}$tumors could be induced into remissions by immunizing them with intra-lymph node injections of an E7 vaccine and treating them with a dsRNA TLR3 ligand (507).

Nitric oxide synthases generate the free radical from L-arginine. The bioreactivity of NO consists of the induction of ss and dsDNA breaks and DNA cross-linkages. In the DNA molecule $\mathrm{G}: \mathrm{C}$ and $\mathrm{T}: \mathrm{A}$ transversions occur resulting in the formation of the mutagenic 8-nitroguanine. The wild-type p53 eliminates damaged cells by inducing their apoptotic death. However, when E6 causes the degradation of p53, and E7 blocked the retinoblastoma protein, the mutated virus-carrier cells survive and replicate. Thus an inflammatory condition with the release of free radicals is co-carcinogenic (508a).

About one third (38\%) of the inflammasomes of actinic keratosis and the non-melanoma skin cancers (basal cell and squamous cell carcinomas) that follow harbor HPV genomic sequences (by PCR assays), but practically none herpesviral (EBV, CMV) sequences (508b).

Viral IV. Hepatitis B and C viruses with or without alcohol and/ or aflatoxin exposure induce hepatocellular carcinoma (HCC), after keeping the liver parenchyma inflamed and becoming cirrhotic for several years (or decades). G1896A and A1762T/ G1764 double mutant HBV induces cirrhosis and HCC. Serum HBV DNA levels may clear to the negative stage of HBeAg and HBsAg. G1776A viral genomic mutations favorably prognosticated the achievement of a negative HBeAg status $(509,510)$. The proteomics of infected hepatocytes in patients with hepatitis B surface antigen (SHBs) expression revealed high rate of apoptotic deaths. Apoptosis-susceptibility or resistance was regulated by the following genes: glucose-regulated protein $78 \mathrm{kDa}$ (GRP78), heterogenous nuclear ribonucleoprotein H3, Rho GDP (guanine diphosphate) dissociation inhibitor, cystatin B, far upstream element-binding protein and TNF receptor associated protein 1 . The gene GRP78 encoded apoptosis-resistance; siRNA treatment silencing this gene resulted in apoptosis susceptibility. Circulating hepatitis B surface antigen (HBsAG) rendered infected hepatocytes susceptible to apoptosis (511).

The HBV genome is directly oncogenic; it is often compared to the retroviral genome's RNA transcribed into proviral DNA by the viral RT. Like in the case of retroviruses, the HBV genomic DNA also integrates into the host cell's genome. The HBV X gene (Hex) and its oncoprotein eliminate the proapoptotic p53 protein, activate methyltransferases to silence more tumor suppressor genes, or gene promoters, by hypermethylation and increase telomerase activity. In summation, it is an anti-apoptosis and anti-senescence agent (512). HBx activates miR-29a, which suppresses PTEN and promotes the migration of transformed liver (hepatocellular carcinoma) cells (513).

Hepatitis $\mathrm{C}$ virus (HCV) enters cells through the integral tight junction protein occludin and its membrane-associated MARVEL domain (MAL proteins, myelin and lymphocytes; MAL-related for vesicle trafficking and membrane link) (514). MARVEL-defective cells were non-permissive to HCV entry. The HCV envelope proteins E1 and E2 interact with the phospholipid scramblase 1 securing attachment of the viral particles (515). Only hepatocytes are susceptible to CV entry and its core proteins (Core 3a) interact with host proteins, the heterogenous nuclear ribonucleoprotein $\mathrm{H} 1$, nuclear factor 45, and C14 open reading frame 166 (516). These interactions trigger the pre-oncogenic pathways in the infected cells. These processes occur against the background of anti-viral immune reactions in the liver parenchyma, which include oxidative stress and neoangiogenesis. Generation of iNOS, Cox-2, VEGF, PGE2 and activation of the Akt pathway occur. Administration of Core $3 \mathrm{a}$ and Cox-2 specific siRNAs reduced all these reactions and the size of the viral progenies released $(517,518)$. HCV genome encodes p7, a peptide functioning as an ion channel in lipid bilayered membranes. RNA interference (siRNA) inhibits the encoding of HCV envelope proteins E1, 2 and thus reduces intracellular viral replication (519abcd). Chronic HCV infection could culminate into MALT lymphoma with $\mathrm{t}(14 ; 18) \mathrm{IgH} /$ Bcl-2 translocation (520).

About $55 \%$ of the patients with $\mathrm{HCV}$ infection treated with pegylated IFN $\alpha$ and ribavirin can achieve sustained viral remissions depending on the viral genotype and the type of the patients' immune response. Of the lympho-cytokine response, steady levels of IL-4 (a Th2-type inducer) and TNFa (a Th1-type inducer) were necessary (521).

Nrf2, the nuclear factor erythroid-2 related factor-2 is activated in response to oxidative stress in HCV-infected cells. MAPK is the activator. Under these circumstances phosphorylated Akt is also activated. Activated Nrf2 and the "cell survival pathways' overcome BAD (Bcl-2 antagonist of cell death) and prolong the life of $\mathrm{HCV}$-infected cells (522). In HCV-induced liver cirrhosis and $\mathrm{HCC}$, TGF $\beta$ induces EMT resulting in invasion and metastasis by the transformed tumor cells (523). Notoriously, NFkB protects HCC from apoptotic death. The protein NEMO (NF essential modulator) activates $\mathrm{NF} \kappa \mathrm{B}$; if it is switched off, $\mathrm{NF} \kappa \mathrm{B}$ remains silent, thus HCCs are allowed to die apoptotic death (524). In patients with advanced pre-cancerous liver cirrhosis, the anti-inflammatory agent colchicine prevented malignant transformation: this occurred in Ciudad Mexico in $9 \%$ of colchicine-treated patients versus in $29 \%$ of untreated patients (525). Previously, treatment with lamivudine, adefovir, tenofovir protected somewhat patients with hepatitis B from HCC advancement (526). Now, surgically non-resectable HCC is being treated with sorafenib or sunitinib, or with oncolytic virus therapy, i.e., genetically engineered adeno-, herpes-, measles-, myxo-, retrolenti-, vaccinia-, and vesicular stomatitis- 
viruses $(164,527-531)$. Sunitinib reduces the number of myeloid suppressor and Treg cells (532). The mechanisms of viral oncolysis directly targeting cancer cells should override any possible adverse effect on inflammatory carcinogenesis.

Bacterial. Helicobacter pylori (Hp) is the classical bacterial pathogen that induces severe, host-damaging inflammatory reactions, while depositing its oncogene cagA (cytotoxin-associated gene product protein) in epithelial cells of the gastric mucosa, or in lymphocytes invading the lesions. Its pathogenesis generated an excessive literature that was recently and repeatedly reviewed in ref. 62 . In a brief summary for a very complex issue (by a clinical oncologist-hematologist-infectious diseases specialist, who has had the opportunity to diagnose and treat patients with $H$. pylori-induced MALT lymphomas): CagA entering lymphocytes and/or gastric mucosal epithelial cells, undergoes phosphorylation, binds Src kinase and $s r c$ homology domain 2-containing tyrosine phosphatase (Rous sarcoma c-src proto-oncogene) and activates extracellular signal-regulated kinase (ERK). The MAPK cascade is activated. In lymphocytes, the anti-apoptotic $\mathrm{Bcl}_{\mathrm{XL}}$ proteins take over. In gastric mucosal cells, H. pylori induces hypermethylation of the $\mathrm{CpG}$ dinucleotides, and deacetylation of histone tailes, and methylations of selected lysine and arginine groups of histones in gene promoters. The inactivation of the gene for RUNX3 (533) by CpG island hypermethylation provided apoptosis-resistance of the involved cells, mediated by $\mathrm{Bcl}-2$ and $\mathrm{Bcl}_{\mathrm{xL}}$ overriding the pro-apoptotic $\mathrm{BAX}$ and BAK proteins (runt-related transcription factor, $\mathrm{B}$ cell lymphoma extra large, Bcl-2-associated $\mathrm{X}$ protein, $\mathrm{Bcl}-2$ antagonist killer) MALT lymphomas are originated in the stomach and elsewhere $(534,535)$. Gene translocations characterize MALT lymphoma cells: $\mathrm{t}(11 ; 18)(\mathrm{q} 21 ; \mathrm{q} 21), \mathrm{t}(14 ; 18)(\mathrm{q} 32 ; \mathrm{q} 21)$ favoring $\operatorname{IgH}$ and Bcl-2 and the activation and release of NFkB. Hp-infected macrophages release a proliferation inducing ligand (APRIL) to B cell receptors (536-538).

The epithelial cells assume goblet cell phenotypes ('intestinal metaplasia'). The cytoplasmic $\beta$-catenin translocates to the nucleus, where it is to activate some proto-oncogenes $(539,540)$. Several CpG islands are methylated in a reversible fashion: most of the sites (not all of them) get demethylated after the eradication of Hp (541). The WWOX tumor suppressor gene (vide supra) is silenced by hypermethylation of its promoter (542). Overriding cell senescence, CagA induces the guanine exchange factor/cMyc/miRNA-p21 ${ }^{\text {waf/cip }}$ axis for driving the cell cycle toward uncontrolled proliferation and EMT, tantamount to local invasion and distant metastasis formation (535). In Germany, a second Hp protein GroEL emerges independently from CagA, as a major contributor to gastric carcinogenesis (543). Lymphocyte- and granulocyte-monocyte-mediated immune reactions are generated, but fail. Reactive oxygen and nitrogen species rise to induce dsDNA breaks. H. pylori-stimulated macrophages in MALT lymphoma release APRIL, the proliferation-inducing ligand (537). Early $\mathrm{H}$. pylori invasion is reversible (with specific antibiotic regimens). In a case, H. pylori infection was disgnosed and was immediately treated, while lymph node biopsy results were not immediately available. Treatment for $H$. pylori infection resulted in rapid regression of lymphadenopathy. Thereafter the lymph node biopsies revealed Burkitt's-like lymphoma EBV-negative with c-Myc rearrangement, but without Bcl-2 translocation (544).
Hungarian clinicians are very interested in those enzyme inhibitors (targeting urease, carbonic anhydrase, $\gamma$-glutamyl transpeptidase, efflux pump inhibitors) that may inhibit the growth of chlarythromycin-resistant strains of $\mathrm{Hp} \mathrm{(545).}$

Genitourinary parasites. Schistosoma haematobium induces squamous cell carcinoma of the urinary bladder in Central and North Africa (Burkina Faso; Cameroon; Egypt, Entebbe, at Lake Victoria, Uganda; Msambweni, Kenya; Southwestern Morocco, Lake Malawi, Nyasa/Niassa, Mozambique and Tanzania; Erinia River, Osun, Nigeria; Niger; Lusaka province, Zambia; lakes and Limpopo River, Zimbabwe). The intermediate host is the snail sp Bulinus (especially B. truncates/truncatus). However, it was in Brazil, where the hemocytes of the snail sp Biomphalaria were found to be self-protective against $S$. mansoni. The snail hemolyph contains amoebocytes and haemocytes; the haemocytes are granulocytes and hyalinocytes. The granulocytes move on psedopods, phagocytose and encapsulate the parasites (546). Eli Metchnikov (ИЛЬЯ ИЛЬИЧ МЕЧНИКОВ 1845 1916) observed the phenomenon of phagocytosis in the belly of Daphnia pulex, whose entire genome has just now been sequenced in full. The innate immune faculties of the snail offer better protection against the cercaria (547), than the united innate and adaptive immune faculties of the human host. The snail phagocytes generate nitrite oxide to kill the parasites. In the human host, tumor cell genomics give no clear explanation of the pathognesis. Genomics (including the mt genome) of the parasites revealed the lines of their phylogenesis, but so far gave little information as to their ability of evading the infested host's defenses $(548,549)$. However, an extraordinary study on the snails' innate immunity as it is activated against the trematodes, revealed that their thioester-containing protein (TEP) regulates the phagocytosis and encapsulation of the parasites. The fibrinogen-related proteins (FREPs) in a specific and variable manner react with polymorphic mucins of the parasites. These reactions are between diversified immune receptors of the host, and antigenic variants of the parasite (550). In the human host, the tumor suppressor gene p53 may or may not be downregulated, but the anti-apoptotic Bcl-2 gene is activated (551). Only the mucinous and signet ring adenocarcinomas expressed intense c-Myc (552). The RB gene was occasionally suppressed; p53 mutations occurred in 57\% of the tumors. Ras gene point mutations, EGFR c-Erb2 amplifications occurred irregularly (553). The detectable genomic changes (p53, H-ras, VEGF) could not be used as prognostic factors as to the outcome of the disease (554). However, p53 mutation and high Ki-67 expression indicated advanced disease and poor prognosis (555). Human cancer cells, especially those of colon carcinoma, overexpress the highly conserved oncofetal antigens TA1/E16 (tumorassociated, embryonic day 16 lymphocyte activating). Protein homologues of these antigens are expressed in schistosoma species (S. mansoni) (556), and as such, they may act as oncogenes in human cancers.

Polo-like kinases (Plk-1-5) encode centrosome spindle formatting proteins in schistosoma and in cancer cells. The patented drug Bl2536 inhibits mitoses in schistosoma and in cancer cells (557).

Opisthorchis viverrini, and Clonorchis sinensis, the liver flukes, induce cholangiocarcinoma of the bile ducts. In the tumor cells Ski/SnoN oncoproteins are highly overexpressed 
(Sloan-Kettering Institute). These are avian retrovirus-related oncoproteins discovered at the Sloan-Kettering Institute, New York. Proto-oncogene v-ski was captured by an avian erythroleukemia retrovirus SKI-T (c-ski $\rightarrow \mathrm{v}-s k i)$. The human c-ski locus maps to 1q22-24. Oncoproteins c-Ski/SnoN (Ski novel protein) are overexpressed in cholangiocarcinoma with or without induction by $O$. viverrini. SnoN binds the Smad complex and repress TGF $\beta$ 's inhibitory effect on cell proliferation, thus acting as an oncogene. SnoN binds the promyelocytic leukemia (PML) protein and they thus stabilize p53; the cell undergoes senescence and apoptosis. SnoN acted as an anti-oncogene (EMBO $\mathrm{J}$ 2889). In cholangiocarcinoma cells, the retinoblastoma (Rb) and p16(INK4) proteins are reduced. Cyclin D1, CDK4 and Smad4 are up-regulated (inhibitor of CDK4, cyclin-dependent kinase; mothers against decapentaplegic, from drosophila; decapentaplegic $=$ TGF $\beta$ ligand $)$. Oncoprotein $\mathrm{c}$-Ski suppresses TGF $\beta R$, as its direct antagonist. Active TGF $\beta$ antagonizes c-Ski by suppressing its neoangiogenic and metastatic potentials. The negative c-Myc regulator tristetrapolin (TTP) is disabled by a $\mathrm{CpG}$ site methylation, thus liberating c-Myc for the suppression of the anti-proliferative effect of TGF $\beta$ (558-566).

Are the notoriously present latent polyomaviruses JC and BK contributory to carcinogenesis in the GU tract? Extending the question to SV40 simian polyomavirus, that was present in some live attenuated poliomyelitis vaccines, and to the Merkel cell carcinoma polyomavirus, these agents are highly suspect contributors to some rare human neuroectodermal cancers expressing the $\mathrm{T}$ oncoprotein and/or integrated viral genomic sequences in the tumor cells' genome. Inflammatory monocytes serve as a reservoir for the Merkel cell polyomavirus (567-572).

\section{Mechanisms of inflammatory carcinogenesis}

Proteomics. Granule-loaded eosinophil granulocytes are expected to exert complex anti-tumor effects wihin inflammasomes (vide supra). In Hodgkin's granulomas the eosinophils fail to subdue the Reed-Sternberg cell (Fig. 2). The eosinophil granulocytes themselves may succumb to malignant transformation in the form of various hypereosinophilic syndromes including eosinophilic leukemia. The fusion inducing protein/ oncogene includes the platelet-derived growth factor receptor $\alpha$ gene (FIP/FDGFR), susceptible to inhibition by imatinib mesylate or dasatinib. Inflammatory defensive cells, be monocytes, granulocytes, lymphoid cells (lymphocytes and NK cells) or mast cells, do readily succumb to malignant transformation. TAMs are often subverted supporters of growth factors to the tumor. Immature dendritic cells (DCs) are often tolerogenic. The presence of $\mathrm{CD}_{123^{+}}$plasmacytoid DCs in breast cancer tissue is associated with poor prognosis (13).

Genetic polymorphisms (single nucleotide polymorphisms, SNP) may bring out the unexpected: a defensive immune reaction may be the inducer of malignant tranformation in these individuals. SNP of the TLR4 or some of the 'inflammatory genes' increases the risk of high grade prostate cancers. Pro-inflammatory IL-1 gene cluster polymorphism increases the risk of gastric cancer induction in the $H$. pylori-infected organ (vide supra). When TLR4 recognizes LPS endotoxin, it signals through MyD88/IL-1 to induce NFKB and the MAPK pathway. Polymorphism of the TLR4 gene may result in defec- tive signaling, lack of defensive reactions and consequentially severe direct tissue damage by the endotoxin (13).

Of damage-associated molecular pattern (DAMP) molecules, high mobility group proteins A and B emerge (HMGAB), as they are released from necrotic cells. The cognate receptors of HMBAB proteins are the RAGE (receptors for advanced glycation end products, glycation, non-enzymatic glycolyzation), or TLR2, TLR4. These receptors generate NFkB, E-selectins and insulin-like growth factor and/or its receptor, IGF-R (selectins are ligands to sialylated cell surface carbohydrates) (13).

How could defensive inflammatory reactions of a host derailed toward carcinogenesis? The promotion of the HER2/ neu oncogene by Freund's adjuvant settled the issue (573). Dormant oncogenes are awakened and activated, when the host mobilizes inflammatory reactions. The importance of chronic inflammatory processes in carcinogenesis is best proven by the anti-oncogenic efficacy of anti-inflammatory agents. The most efficient anti-inflammatory and oncogenesis inhibitory agents are ethyl pyruvate, non-steroidal anti-inflammatory drugs (acetyl salicylic acid, ibuprofen), prostaglandin- and COX2inhibitors (celecoxib) (13).

The human nitric oxide synthase gene is up-regulated by the Wnt/ $\beta$-catenin/Tcf4 signaling ( $\mathrm{T}$ cell factor/lymphoid enhancer factor). TNF $\alpha$, IL-1 $\beta$ and IFN $\gamma$ induce nitric oxide expression (hiNOS) by acting on the NOS gene promoter. NFKB is suppressed by $\beta$-catenin. The summation of these reactions is a decrease of hiNOS production in colon cancer and hepatocellular cancer cell lines (574).

Autophagy. Reduced HMG proteins induce mitochondrial superoxide production promoting autophagic cells. Other inducers of autophagy are perifosine (an mTOR inhibitor), and TGF $\beta$ (a multifunctional cytokine) $(575,576)$. In autophagic cells, induced by autophagy genes, the cell survival pathway PI3K and the pro-apoptotic beclin (the mammalian ortholog of yeast autophagy gene, $a t g$ ) compete; $\mathrm{Bcl}-2 / \mathrm{Bcl}_{\mathrm{XL}}$ antagonize beclin (577). Autophagic tumor cells may recover as progressive tumors; this can be inhibited by hydroxychloroquine (578). Autophagy may protect tumor cell during genotoxic and metabolic stress. However, resveratrol and curcumin may induce tumor cell death in autophagy and in mitotic catastrophe (579-581). The mitochondrial alternating reading frame, p14 ${ }^{\mathrm{ARF}}$ exerts anti-tumor effects by antagonizing MDM2, thus protecting $\mathrm{p} 53$, and suppressing c-myc/MYC. In autophagic cells, p14 ${ }^{\mathrm{ARF}}$ removes the Beclin/ $\mathrm{Bcl}_{\mathrm{XL}}$ complex and suppresses tumor cell recovery from autophagy (582). Inflammatory events in the tumor environment may induce autophagy of tumor cells as a stress adaptation response (583ab).

Autoimmunity. The epigenomics of EBV $(474,584,585)$, and reactivated endogenous retroviruses definitely are major contributors to the etiology of autoimmunity, especially in lupus erythematosus and multiple sclerosis (reviewed in ref. $178,474,586)$.

While the omnipresent cytoplasmic filamentous structures were not unenveloped myxo-, or retroviral strands (587ab,588), but products of the endoplasmic reticulum in response to IFN $\alpha \beta$ oversecretion, serological and biochemical evidence for a herpesvirus (EBV) triggering the maturation of endogenous 
retrovirus(es) is becoming steadily more convincing throughout of the passing decades $(62,152,474)$.

One would expect that the chronic (but self-directed) immune reactions would increase the incidence of malignant tumors, especially those of the lymphatic system. Increased lymphomagenesis in patients with autoimmune diseases (lupus) is well confirmed. The incidence of parotid gland marginal zone lymphoma in patients with Sjøgren's syndrome is 1000fold increased. In SLE, lymphomagenesis is minimally (3-fold) increased (589). Further, it is suspected, but not proven, that EBV in the background, and sporadic buddings of endogenous retroviruses in the lymphoma cells in the foreground, are the etiological agents (178). Unexpectedly, there is lower incidence of adenocarcinomas (breast, colon, prostate) against the background of a bona fide autoimmune disease, treated or untreated (590,591). The German Cancer Research Center in Heidelberg suggests that the natural risk for cancers in autoimmune diseases is increased, but is artificially reduced by suppressing the inflammatory component of cancer induction by antiinflammatory medications (592). MicroRNA-155 emerges as one of the master regulators of immune cell generation, as it has the ability to target mRNAs of a wide variety of the cellular immune system. Should a lymphoid cell population turn malignant, miR-155 may be able to suppress its mRNAs (593).

The IL-17+CD4 ${ }^{+}$Th17 lymphocytes are known to mediate autoimmune reactions. A stimulatory role to anti-tumor immune $\mathrm{CD}^{+} \mathrm{T}$ cells of a Th17+ lymphocyte subpopulation was recognized; chemokines CCL2 and CCL20 and IL-2 mobilized this population of Th17 lymphocytes, which did not have the ability to directly kill tumor cells (594). An $\mathrm{IL}_{-17} 7^{+} \mathrm{CD} 4^{+} \mathrm{FoxP} 3^{+}$regulatory Th17 cell population forms the minority of Treg cells. This population of Th17 cells derived from $\mathrm{CCR}^{+}$memory $\mathrm{T}$ cells under the effect of myeloid antigen-presenting cells, IL-2 and TGF $\beta$. In the colon, the CD $4^{+}$Fox $3 \mathrm{P}^{+} \mathrm{Th} 17$ lymphocyte population suppressed $\mathrm{CD}^{+} \mathrm{T}$ cells, and induced inflammatory cytokines. The Th17reg cells promote ulcerative colitis and thus colon carcinogenesis (595).

Reactive oxygen species and related genotoxic events. Positive comments on inflammatory carcinogenesis based on dsDNA damage (breaks, mutations) by reactive oxygen and nitrogen species abound in the literature (596-598). Followers of Professore Flaviano Magrassi, distinguished member of the 'Accademia Nazionale dei Lincei', from Naples, Italy, state 'A mutated cell is a sine qua non for carcinogenesis' (596). Hypoxia, DNA damage and genome instability; DNA breaks misrepaired (in the form of fusions of distant genes); aging and cancer; the necessity of $\mathrm{G}$ protein-coupled receptor for $\mathrm{p} 53$-dependent cell survival in response to genotoxic stress; nicotinamide phosphoribosyltransferase: a molecular link between metabolism, inflammation and cancer; aldehyde dehydrogenase-expressing colon stem cells contributing to tumorigenesis in the transition from colitis to cancer; high mobility group A1 gene: transforming inflammatory signals into cancer; oxidative stressing in prostate cancer is required for aggressive phenotype; role of IAPs in NFKB activation by genotoxic stresses; mitochondrial dysfunction and reactive oxygen species imbalance promote breast cancer motility through CXCL14; tyrosine kinase c-Src in the regulation of reactive oxygen species generation; role of mismatch repair in the control of oxidative DNA damage; genetic instability and tumorigenesis; induction of epigenetic alterations by chronic inflammation and its significance in carcinogenesis, are the long list of topics recommended for review from the Epub service. Indeed, a high innate production of pro-inflammatory cytokines was a signal for increased risk for death from cancer (599).

Oxidative DNA damage occurs consequentially to 'leaks' from electron transport chains. Leak electrons acting with oxygen produce superoxides often released in bursts. Certain enzymes also generate superoxides released in bursts. This is the established practice of phagocytic cells for the destruction of pathogens. Peroxisomes practice normal oxidative metabolism. The unintentional oxidative damage to DNA is caused by the highly reactive hydroxyl radicals abstracting $\mathrm{H}$ atoms from the methyl groups $\left(\mathrm{CH}_{3}\right)$ of thymine and from the $\mathrm{C}-\mathrm{H}$ bonds of 2'-deoxyribose. Additions to the C5-C6 double bond of pyrimidines transforms these into $\mathrm{C} 5-\mathrm{OH}$ and $\mathrm{C} 6-\mathrm{OH}$ adduct radicals. $\mathrm{H}$ atom abstraction from thymine forms an allyl radical. The $\mathrm{C} 5-\mathrm{OH}$ adduct radicals are reducers, and the $\mathrm{C} 6-\mathrm{OH}$ adduct radicals are oxidizers. Addition of oxygen to $\mathrm{C} 5-\mathrm{OH}$ adduct radicals yield C5-OH-6-peroxyl radicals. C5-OH-6-peroxyl radicals eliminate $\mathrm{O}_{2} \cdots$; after reaction with water, thymine and cytosine glycols are formed. Allyl radicals reacting with oxygen form 5-hydroxymethyluracil and 5-formyluracil. Thymine peroxyl radicals are reduced to hydroxyhydroperoxides decomposing into thymine glycol, and 5-hydroxyl- and 5-formyluracyls and hydroxyl-methylhydantoin. In certain early stages, oxidative DNA damage can be repaired. Genome repair begins with the enzymatic reactions: glycolases removing single lesions, or enzymes excising nucleotides. These are highly specific processes, like the removal of purin-derived, or pyrimidinederived oxidative lesions. The repair may be defective, but the DNA remains functional. For example, deamination of cytosine to uracil is a pro-mutagenic mistake (600). The glutathione peroxidase gene (GPX1) in the short arm of chromosome 3 frequently suffers loss of heterozygosity $(\mathrm{LOH})$, thus producing a defective enzyme, the gene product protein peroxide scavenging enzyme (600).

These events occur when the repair genes suffered SNP (vide supra). In response, mammalian cells express stress-induced genes encoding antioxidant reactions. However, further reactions along these lines leave the DNA molecule either mutated or non-functional. The scientific community hypothesizes that if the mutated cells are not eliminated by the 'guardian of the genome', the WTp53 pro-apoptotic machinery, mismatched repairs and gene fusions may resurrect the damaged cell in a shape and condition not fit into the orderly operation of a multicellular organisms, as if the resurrected cell would not fit into an organized cell community, but could live as a unicellular organism on its own. For example in a tissue culture vessel nourished and cleansed. Under these circumstances it would prove itself to be exempt of senescence and natural death. Its DNA would dictate incessant cell divisions 'forever'. This author, growing $\mathrm{EBV}^{+}$human lymphoblasts in suspension cultures and sarcoma and melanoma cells in solid cultures for over a decade, wrote in 1976 (321,601a): 'Could the cancer cell be viewed as an individual that extricated itself from boundaries exposed (imposed) on it by 500 millions years ( 3 billion) of evolution and recaptured the immortality of unicellular organisms that existed before the social order of cell communities was estab- 
lished? Certainly, some cell cultures of mammals, including man, create the impression of immortality of archaic unicellular life forms (i.e., lymphoblastic cells grown in suspension culture as long as nutrients are provided and metabolic waste is removed)' (601a). However, if the resurrected cell remains in its cell community, it may be attacked and killed. It may be able to evade such attacks and continue to replicate and eventually consume its host, as if it were an alien invader (601a).

'Demonstrating a link between defects in repair of oxidative DNA damage and a propensity for disease has not been easy'. Carcinogenesis probably requires combined gene mutations or knock-outs. 'The mere presence of damage is not proof of a causative link' (600), a scientifically justified most cautious comment in 2003; by 2010 the evidence for a positive relationship between inflammatory processes and carcinogenesis has reached an overwhelming volume and quality (599).

The empress (RNA) and the emperor (DNA) of all molecules. The ancient RNA/DNA interactions continue in the most complex intracellular environments. The position of the stem cell gene Sox 2 residing embedded in a long non-coding RNA strand (359), strongly suggests that such constellations might have already been formed in the precellular era on Earth. The microRNAs retain the power over DNA as they are capable of silencing the mRNAs of DNA-derivation. Actually, the microRNAs cooperate with the DNA, when the stem cell- or somatic cell-serving DNA genome transforms itself into a set of multiple oncogenes, thus preventing the cells' senescence and death. It may appear as if the microRNAs dictated the reversal of the cell-serving DNA into its primordial 'immortal' format (vide infra). The stem cells may rest, self-renew, yield daughter cells for specific differentiation in the form of functional somatic cells, or transform toward immortalized cell populations, whereby the DNA genome undergoes ds breaks; misrepaired, the dsDNA breaks result in gene fusions and oncogene formations, while genes inhibitory to this process (the 'tumor suppressor genes') are deleted. Inflammatory processes frequently signal the stem cell (or the differentiated somatic cell) toward the latter pathway. It appears as if microRNAs guide the DNA toward which way to go. Thus inflammatory reactions indirectly through miR-mediation penetrate the epigenome and act through its modification. A scholarly review spreads the word in Hungarian (so convincingly, as if the crosstalk between RNA and DNA actually went on in Hungarian) (601b). Indeed, the ancient RNA/DNA crosstalk should be understood in all languages. It is spelling out the verdict of life or death for the cell, from the time on, since cells lived on Earth. The extraordinary versatility of the DNA in its transformation into sets of oncogenes and its interaction with the epigenome in the process was well recognized even before the discovery of the microRNAs (602).

The question arises if it is not so, that hyperthermia (HSF), hypoxia (HIF), reactive oxygen/nitrogen species (ROS, iNOS) and stress, and showers of inflammatory cytokines release their danger signaling to the RNA/DNA complex. The somatic cellserving DNA in multicellular organisms accepted senescence and death, but retained suppressed but preserved, its primordial faculties of independence and 'immortality' in the germ cells and in the stem cells. The haploid germ cells in their unisons become differentiating somatic cells. However, the self-renewing stem cells, instead of the route to differentiation, may choose a reversal toward a primordial stage of life: restoring the ancient DNA and the ways it existed under precellular conditions intimately reacting with RNA and with 'amorphous proteins', as amino acid chains 'self-replicating' $(603,604)$. In the first cellular life forms, DNA was highly resistant to physicochemical damage and voraciously accepting for fusion and recombination alien genomic sequences (62). Upon the encounter of amorphous proteins and naked DNA, the proteins engulf the DNA and DNA strands penetrate to the helices of the proteins, while the proteins undergo subtle morphological changes of their nanoparticulate architecture (604). Innumerable such newly formed structures may exist in nature without being able to enter cells for replications, inasmuch as the natural proteins can not serve as specific ligands to any cell receptors (whereas, the proteins generated in the Domingo-Espin laboratory were actually encoded by borrowed viral genes from the foot and mouth virus and from the SV40 virus, and thus have become 'artificial viruses' able to react with cell and nuclear membrane receptors and transgress them) (604). In contrast, the natutally formed entities may show up in the metagenomic samples (in a mixture with regular incomplete virions), as the 'gene transfer agents' of Kristensen et al (605), or among the structures considered to form a 'fourth domain of life' on Earth (606).

The spheroplasts of a crenarchaeota and a prokaryota might have been fused by a fusogenic phage of an ancestral mycoplasma (like that of the Acheloplasma $\mathrm{sp}$ ) to form the first eukaryotic cells (an experiment of Nature that could be repeated in the lab today) (607). Since their origin by cell fusion, eukaryotic cells continue to readily communicate through cell fusions in physiological or oncogenic terms; all oncogenic viruses are able to fuse cells $(62,608,609)$. Lymphoma cells naturally fused with specific antibody-producer plasma cells (referred later to as 'natural hybridomas') discovered in mice in the late-1960's are presumed to exist in human patients $(62,225,610-614)$. In the first unicellular nucleated eukaryotes, with or without the acquisition of mitochondria or chloroplasts, the RNA/DNA complex maintained its superiority over all other molecules, either within the cell, or outside the cell, as phages converted into RNA ('the burst' of picornaviruses) (615), and into DNA eukaryotic viruses (616). Those sophisticated cells divided before senescence and death; natural death was not encrypted in their DNA genomes. In adverse circumstances, the most versatile ancient DNA transformed them into morphological and physiological entities grossly different from their basic original life forms: as if into a different (from an a-flagellate to a flagellate) species. The Naegleria DNA still practices such transformations of its host $(617,618)$. Later, in the unicellular parasite Theileria under immunological attack in an invaded bovine host, the most versatile theileria DNA encodes an elaborate physiology for the parasite to evade that immunological attack from its host (619). In the theileria-induced fatal lymphoproliferative disease, the East Coast fever, the parasite induces NFKB to secure apoptosis-free state of the schizont-infected lymphocytes. Theileria-infected macrophages bring into existence an autocrine loop for TGF $\beta 2$ and induce MMP9 for their survival and invasiveness $(620,621)$, thus using the same molecular mechanisms cancer cells utilize for their survival and invasiveness. In the glioblastoma cell, the DNA can switch from the anaerobic glycolysis to oxidative phosphorylation and metabolism (622). 
The Namalwa Burkitt's lymphoma cell DNA under attack with heavy irradiation, suffers severe ds breaks. The $\gamma \mathrm{H} 2 \mathrm{AX}$ (ataxia) histone detects an extraordinarily high incidence of these ds breaks. However, the oncogenic DNA (with the help of repair enzymes like the recombinase Rad51, forming nucleoprotein complexes from ss/ds broken DNA sequences) recovers from the 'mitotic catastrophe' (623). In the enzymefree (except for the ribozymes) pre-cellular RNA/DNA world, ss or dsRNA/DNA breaks might have been repaired telomerefree by end-to-end seals (which are chromosome fusions in the celluler world) (624).

The existence of viral genes related to RNA/DNA replication, but not shared with cellular genes (the 'viral hallmark genes' of Koonin et al, vide infra) strongly suggests that the origin of these genes predates the appearance of protocells (625). For self-replicating macromolecules in hypercycles (a symbiosis of molecules self-replicating in hypercycles, Ghadiri et al, vide infra), the Eigen's error catastrophe phase transited into a thermodynamic phase governing templated RNA/DNA synthesis (626).

Following the ideas of old-time astute observers, Arguello proposes that the cancer cell is the result of an 'atavistic metamorphosis' (627a). Duesberg et al (627b) consider the cancer cell to be the product of a trans-speciation process.

The oncogenic DNA bioengineers the neoplastic cell as its ancestors could (and still do) bioengineer the naegleria cell, while it remains close to indestructible, as its predecessor, the primordial DNA, must have been (628). Are oncogenes representing a reversal of the cell-serving DNA to its primordial format? Is the multiply fused and most powerful oncogenic DNA a replica of the primordial DNA? Is it not so, that the malady that we diagnose as 'cancer', is the expression of an inherent fundamental attribute of the DNA, its reversal from the differentiated to the native undifferentiated format in order to sustain life under any conditions and in any shape (628)? Were it not our killer, scientists would admire the cancer cell and its DNA 'oncogenes' as the ultimate achievement of bioengineering.

A 'precellular virus world' and a 'primordial gene pool' could have formed on Earth (629). That world can not only be imagined, but could possibly be duplicated in the laboratory. How the naked DNAs interacted with amorphous amino acid clusters $(603,604)$, hypercycling RNAs in the hydrothermal vents at the bottom of the oceans (and in their laboratory reactors) (630), the catalytic hammerhead ribozymes (631), and the circular self-replicating ('rolling circle') viroid RNAs (preserved from the pre-cellular world in extant plant and mammalian cells, the hepatitis $\delta$ viroid, some with a tandemly repeated homologous DNA attached) (632-635)? In order to envision these interactions in a most hostile precellular environment without potent protein enzymes, that were not being able to form without cellular ribosomes and without a genetic code, both advanced knowledge in proteomics and genomics, and a great deal of imagination, are required. This author for one, subscribes with gratitude the review, Professor W. Ford Doolittle (Dalhouise University, Halifax, Nova Scotia, Canada) rendered, to Koonin et al: 'Virus-like entities surely predated the appearance of modern cells...Evolutionary scenarios are an artform. They usefully exercise the brain...They do not have to be true! I do not disfavor the publication...' (629).

\section{Acknowledgements}

The author's views toward the idea of infectious and inflammatory carcinogenesis were profoundly influenced by Dr Milton Wainwright, senior lecturer in microbiology at the University of Sheffield, England, both in personal conversations and by reading of his writings. Further recommended reading is the volume 'Microbes and Malignancy. Infection as a cause of cancers' edited by Julie Parsonnet (Oxford University Press 1999). The author thanks to Professor János Minárovits, President of the Hungarian Microbiological Society, for allowing the full presentation of this material at the 16th International Congress of the Hungarian Society for Microbiology, July 20-22, 2011, Budapest, Hungary. The author expresses his gratitude to Professor Károly Nagy, Chairman of the Institute of Medical Microbiology, Semmelweis University, Budapest, Hungary, for his invitation to the author to present this material to a distinguished audience on May 12, 2011.

\section{References}

1. Balkwill $\mathrm{F}$ and Mantovani A: Inflammation and cancer: back to Virchow? Lancet 357: 539-545, 2001.

2. Editorial, 'Suffering Man, Isenheim Altarpiece'. Clinical Infectious Diseases 47: Cover and text, Sept 15, 2008.

3. Howe CD and Sinkovics JG: Life-threatening infections (endotoxin shock included) in patients with cancer. Project M26/ gm 9. Research Report, The University of Texas M.D. Anderson Hospital, Houston, TX. Printing Division, The University of Texas, Austin, TX 316-320, 1967; 303-305, 1970; 486-494, 1972; 465-466, 1975.

4. Sinkovics JG: Clinical recognition and treatment of endoxinemia. In: Immunobiology and Immunopharmacology of Bacterial Endotoxins. Szentivanyi A and Friedman H (eds). Plenum Press, pp269-279, 1986.

5. Sinkovics JG, Gröschel D, Plager C, Howe CD, Martin R and Smith J: Clinical observations in severe infections and a study of immunological defects involved. Project M27/gm 12. Research Report, The University of Texas M.D. Anderson Hospital, Houston, TX. Printing Division, The University of Texas, Austin, TX 532-533, 1976; 327-328, 1978.

6. Sinkovics JG: Infectious complications. In: Medical Oncology an Advanced Course. Volume II. Marcel Dekker, NY, pp1557-1739, 1986.

7. Li Y and Alam HB: Modulation of acetylation: creating a prosurvival and anti-inflammator phenotype in lethal hemorrhagic and septic shock. J Biomed Biotechnol 2011: 523481, 2011.

8. Leu JI, Pimkina J, Frank A, Murphy ME and George DL: A small molecule inhibitor of inducible heat shock protein 70 . Mol Cell 36: 15-27, 2009.

9. Leu JI, Pimkina J, Pandey P, Murphy ME and George DL: HSP70 inhibition by the small-molecule 2-phenylethynesulfonamide impairs protein clearance pathways in tumor cells. Mol Cancer Res doi: 10.1158/1541-7786.MCR-11-0019, 2011.

10. Wood J, Pring M, Eveson JW, Price N, Proby CM and Haque A: Co-expression of Bag-1 and heat shock protein 70 in human epidermal squamous cell carcinoma: Bag-1-mediated resistance to 5-fluorouracil-induced apoptosis. Br J Cancer 104: 1459-1471, 2011.

11. Sims JD, McCready J and Jay DG: Extracellular heat shock protein (Hsp) 70 and $\mathrm{Hsp} 90 \alpha$ assist in matrix metalloproteinase-2 activation and breast cancer cell migration and invasion. PLoS One 6: e18848, 2011.

12. Schildkopf P, Frey B, Ott OJ, Rubner Y, Multhoff G, Dauer R, Fietkau R and Gaipl US: Radiation combined with hyperthermia induces HSP70-dependent maturation of dendritic cells and release of pro-inflammatory cytokines by dendritic cells and macrophages. Radiother Oncol 2011 [Epub ahead of print].

13. Lyn-Kew K, Rich E, Zenf X, Wen H, Kunkel SL, Newstead MW, Bhan U and Standiford TJ: IRAK-M regulates chromatin remodeling in lung macrophages during experimental sepsis. PLoS One 5: e11145, 2010. 
14. Arcaroli J, Silva E, Maloney JP,He Q, Svetkauskaite D, Murphy JR and Abraham E: Variant IRAK-1 haplotype is associated with increased nuclear factor- $\mathrm{\kappa B}$ activation and worse outcome in sepsis. Am J Respir Crit Care Med 173: 1335-1341, 2006.

15. Demaria S, Pilarsky E, Karin M, Coussens LM, Chen Y-C, El-Omar EM, Trinchieri G, Dubinett SM, Mao JT, Szabo E, Krieg A, Weiner GJ, Fox BA, Coukos G, Wang E, Abraham RT, Carbone $\mathrm{M}$ and Lotze MT: Cancer and inflammation: promise for biological therapy. J Immunother 33: 335-351, 2010.

16. Lange SS, Mitchell DL and Vasquez KM: High mobility group protein B1 enhances DNA repair and chromatin modification after DNA damage. Proc Natl Acad Sci USA 106: 10320-10325, 2008.

17. Yang R, Tan X, Thomas AM, Shen J, Qureshi N, Morrison DC and van Way CW III: Crocetin inhibits mRNA expression for tumor necrosis factor-alpha, interleukin-lbeta, and inducible nitric oxide synthase in hemorrhagic shock. J Parenter Enteral Nutr 30: 297-301, 2006

18. Sun Y, Varambally S, Maher CA, Cao Q, Chockley P, Toubai T, Malter C, Nieves S, Tawara I, Wang Y, Ward PA, Chinnaiyan A and Reddy P: Targeting of microRNA-142-3p in dendritic cells regulates endoxin-induced mortality. Blood 117: 6172-6183, 2011

19. Domer PH, Fakharzadeh SS, Chen CS, Jockel J, Johansen L, Silverman GA, Kersey JH and Korsmeyer SJ: Acute mixed lineage leukemia $\mathrm{t}(4 ; 11)(\mathrm{q} 21 ; \mathrm{q} 23)$ generates an MLL0-AF4 fusion product. Proc Natl Acad Sci USA 90: 7884-7888, 1999.

20. Harper DP and Aplan PD: Chromosome rearrangement leading to MLL gene fusions: clinical and biological aspects. Cancer Res 68: 10024-10027, 2008

21. Bueno C, Montes R, Catalina P, Rodrigues R and Menendez P: Insights into the cellular origin and etiology of the infant pro-B acute lymphoblastic leukemia with MLL-AF4 rearrangement. Leukemia 25: 400-410, 2011

22. Stein RA: Epigenetics - the link between infectious diseases and cancer. JAMA 305: 1484-1485, 2011.

23. Iliopoulos D, Hirsch SA and Struhl K: An epigenetic switch involving NF-kappaB, Lin28, Let-7 microRNA, and IL6 links inflammation to cell transformation. Cell 139: 693-706, 2009.

24. Iliopoulos D, Jaeger SA, Hirsch HA, Bulyk ML and Struhl K STAT3 activation of miR-21 and miR-181-b-1 via PTEN and CYLD are part of the epigenetic switch linking inflammation to cancer. Mol Cell 39: 493-506, 2010.

25. Enders GH: Gauchos and ochos: a Wee1-cdk tango regulating mitotic entry. Cell Div 5: 12, 2010.

26. Tili E, Michaille JJ, Wernicke D, Alder H, Costinean S, Volinia S and Croce CM: Mutator activity induced by microRNA-155 (miR-155) links inflammation and cancer. Proc Natl Acad Sci USA 108: 4908-4913, 2011.

27. Butz H, Likó I, Czirják S, Igaz OP, Khan MM, Zivkovic V, Bálint K, Korbonits M, Rácz K and Patócs A: Down-regulation of WEE1 kinase by a specific subset of microRNA in human sporadic pituitary adenomas. J Clin Endocrinol Metab 95: E181-E191, 2010

28. Ross JS: Measuring circulating miRNAs: the new 'PSA' for breast cancer? Oncologist 15: 656, 2010.

29. Makarova KS, Wolf Y, van der Oost and Koonin EV: Prokaryotic homologs of Argonaute proteins are predicted to function as key components of a novel system of defense against mobile genetic elements. Biol Direct 4: 29, 2009.

30. Diederichs S, Jung S, Rothenberg SM, Smolen GA, Mlody BG and Haber DA: Coexpression of Argonaute-2 enhances RNA interference toward perfect match binding sites. Proc Natl Acad Sci USA 105: 9284-9289, 2008.

31. Gu S, Jin L, Zhang F, Huang Y, Grimm D, Rossi JJ and Kay MA Thermodynamic stability of small hairpin RNAs highly influences the loading process of different mammalian Argonautes. Proc Natl Acad Sci USA 108: 9208-9213, 2011.

32. Kim DH and Rossi JJ: RNAi mechanisms and application. Bio Techniques 44: 613-616, 2008.

33. Hu-Lieskovan SD, Heidel JD, Bartlett DW, Davis ME and Triche TJ: Sequence-specific knock-down of EWS-FLI1 by targeted nonviral delivery of small interfering RNA inhibits tumor growth in a murine model of metastatic Ewing's sarcoma. Cancer Res 65: 8984-8992, 2005

34. Eifler AC and Thaxton C: Nanoparticle therapeutics: FDA approval, clinical trials, regulatory pathways, and case study. Methods Mol Biol 726: 325-338, 2011.

35. Davis ME: The first targeted delivery of siRNA in humans via a self-assembling, cyclodextrin polymer-based nanoparticle: from concept to clinic. Mol Pharm 5: 659-668, 2009.
36. Esther RJ,Lamps L and Schwartz HS: Marjolin ulcers: secondary carcinomas in chronic wounds. J South Orthop Assoc 8: 181-187, 1999.

37. Mishra D and Raji MA: Squamous cell carcinoma occurring at site of prior herpes zoster of the scalp: case report of Marjolin ulcer. J Am Geriatr Soc 52: 1221-1222, 2004

38. Smidt LS, Smidt LF, Chedid MD, Bravaresco CS and Chedid MF: Radical surgical treatment for Marjolin ulcer occurring after chronic osteomyelitis. South Med J 98: 1053, 2005.

39. Agullo FJ, Santillan AA and Miller WT: Image of the month Marjolin ulcer. Arch Surg 141: 1143-1144, 2006.

40. Calikapan GT, Akan M, Karaca M and Akoz T: Marjolin ulcer of the scalp: intruder of a burn scar. J Craniofac Surg 19: 1020-1025, 2008.

41. Tank J, Scharschmidt T and Weiner SD: Development of bilateral low extremity Marjolin ulcers after childhood burns. Am J Orthop 37: E113-E115, 2008

42. Katz RD and Goldberg NH: Marjolin ulcer arising within hidradenitis: a case report and literature review. Ann Plast Surg 62: 173-174, 2009.

43. Grewal NS, Wan DC, Roostaeian J and Rubayi SR: Marjolin ulcer in hidradenitis suppurativa: case reports. Ann Plast Surg 64: 315-317, 2010.

44. Tiflikcioglu YO, Ozek C, Bilkay U and Akin Y: Marjolin ulcers arising in extremities. Ann Plast Surg 64: 318-320, 2010.

45. Huang CY, Feng CH, Hsiao YC, Chuang SS and Yang JY: Burn scar carcinoma. J Dermatol Treat 21: 350-356, 2010.

46. Sharma A, Schwartz RA and Swan KG: Marjolin's warty ulcer. J Surg Oncol 103: 193-195, 2011

47. Mertz KD, Schmid M, Burger B, Itin P, Palmedo G, Schärer L Kutzner H, Fernández F, Gueraz MT, Cribler B, Pfaltz M and Kempf W: Detection of Merkel cell polyomavirus in epidermodysplasia veruciformis-associated skin neoplasms. Dermatology 222: 87-92, 2011

48. Zur Hausen S: Merkel cell polyomavirus in the pathogenesis of non-melanoma skin cancer. Pathologe 30 (Suppl 2): 217-220, 2009.

49. Dworkin AM, Tseno SY, Allain DC, Iwanofu OH, Peters SB and Toland AE: Merkel cell polyomavirus in cutaneous squamous cell carcinoma of immunocompetent individuals. J Invest Dermatol 129: 2868-2874, 2009.

50. Tezal M, Sullivan MA, Hyland A, Marshall JR, Stoler D, Reid ME, Loree TR, Rigual NR, Merzianu M, Hauck L, Lillis C, WactawskiWende J and Scannapieco FA: Chronic periodontitis and the incidence of head and neck squamous cell carcinoma. Cancer Epidemiol Biomarkers Prev 18: 2406-2412, 2009.

51. Tezal M, Sullivan MA, Reid ME, Marshall JR, Hyland A, Loree T, Lillis C, Hauck L, Waclawski-Wende J and Scannapieco FA: Chronic periodontitis and the risk of tongue cancer. Arch Otolaryngol Head Neck Surg 133: 450-454, 2007.

52. Horewicz VV, Feres M, Rapp GE, Yasuda V and Cury PR: Human papillomavirus-16 prevalence in gingival tissue and its association with periodontal destruction: a case-control study. J Periodontol 81: 562-568, 2010.

53. Yakob M, Söder B, Meurman JH, Jogestrand T, Nowak J and Söder PO: Prevotella nigrescens and Porphyromonas gingivalis are associated with signs of carotid atherosclerosis in subjects with and without periodontitis. J Periodontal Res doi: 10.1111/j.16000765.2011.01398.x, 2011

54. Mäntylä P, Stenman M, Paldanius M, Saillu P, Sorsa T and Meurman JH: Chlamydia pneumoniae together with collagenase-2 (MMP-8) in periodontal lesions. Oral Dis 10: 32-38, 2004a.

54. Hernádi K, Szalmás A, Mogyorósi R, Czompa L, Veress G, Csoma E, Márton I and Kónya J: Prevalence and activity of Epstein-Barr virus and human cytomegalovirus in symptomatic and asymptomatic apical periodontitis lesions. J Endod 36: $1458-1459,2010 \mathrm{~b}$

55. Jotwani R, Eswaran SV, Mounga S and Cutler CW: MMP-9/ TIMP-1 imbalance induced in human dendritic cells by Porphyromonas gingivalis. Immunol Med Microbiol 58: 314-321, 2010.

56. Groeger S, Doman E, Chakraborty T and Meyle J: Effects of Porphyromonas gingivalis infection on human gingival epithelial barrier function in vitro. Eur J Oral Sci 118: 582-589, 2010.

57. Groeger S, Domann E, Gonzales JR, Chakraborty T and Meyle J: B7-H1 and B7-DC receptors of oral squamous carcinoma cells are upregulated by Porphyromonas gingivalis. Immunobiology 2011 [Epub ahead of print]. 
58. Fukaya T, Takagi H, Sato Y, Sato K, Eizumi K, Taya H, Shin T, Chen L, Dong CA, Szuma M, Yagita H, Malissen B and Sato K: Crucial roles of B7-H1 and B7-DC expressed on mesenteric lymph node dendritic cells in the generation of antigen-specific $\mathrm{CD} 4^{+}{ }^{\mathrm{F}}$ ox $\mathrm{P}^{+}$regulatory $\mathrm{T}$ cells in the establishment of oral tolerance. Blood 116: 2266-2276, 2010.

59. Tseng S-Y, Otsuji M, Gorski K, Huang X, Slansky JE, Pai SI, Shalabi A, Shin T, Pardoll DM and Tsuchiya H: B7-DC, a new dendritic cell molecule with potent costimulatory properties for T cells. J Exp Med 193: 839-846, 2001.

60. Brandl C, Ortler S, Herrmann T, Cardell S, Lutz MB and Wiendl H: B7-H1-deficiency enhances the potential tolerogenic dendritic cells by activating CD1d-restricted type II NKT cells. PLoS One 5: e10800, 2010

61. Hansen JD, Du Pasquier L, Lefranc MP, Lopez V, Benmansour A and Boudinot P: The B7 family of immunoregulatory receptors: a comparative and evolutionary perspective. Mol Immunol 46: 457-472, 2009

62. Sinkovics JG: Horizontal gene transfers with or without cell fusions in all categories of the living matter. In: Cell Fusions in Health and Disease. II. Cell Fusions in Disease. Dittmar T and Zänker KS (eds). Springer Verlag. Adv Exp Med Biol 714: 5-69, 2011.

63. Nohata N, Sone Y, Hanazawa T, Fuse M, Kikkawa N, Yoshino H, Chiyomaru T, Kawakarni K, Enokida H, Nakagawa M, Shozu M, Okamoto $\mathrm{Y}$ and Seki N: miR-1 as a tumor suppressive microRNA targeting TAGLN2 in head and neck squamous cell carcinoma. Oncotarget 2: 29-42,2011a.

63. Nohata N, Hanazawa T, Kikkawa N, Sakurai D, Sasdaki K, Chiyomaru T, Kawakami K, Yoshino H, Enokida H, Nakagawa M, Okamoto Y and Seki N: Identification of novel molecular targets regulated by tumor suppressive $\mathrm{miR}-1 / \mathrm{miR}-133 \mathrm{a}$ in maxillary sinus squamous cell carcinoma. Int J Oncol 39: 1099-1107, $2011 b$.

64. Nohata N, Hanazawa T, Kikkawa N, Mutallip M, Sakurai D, Fujimura L, Kawakami K, Chiyomaru T, Yoshino H, Enokida H, Nakagawa M, Okamoto Y and Seki N: Tumor suppressive microRNA-375 regulates oncogene AEG-1MTDH in head and neck squamous cell carcinoma (HNSCC). J Hum Genet 56 : 595-601, 2011 .

65. Schmidtner J, Distel LV, Ott OJ, Nkenke E, Sprung CN, Fietkau R and Lubgan D: Hyperthermia and irradiation of head and neck squamous cancer cells causes migratory profile changes of tumor infiltrating lymphocytes. Int J Hyperthermia 25: 347-354, 2009.

66. Young MR, Santhanam AN, Yoshikawa N and Colburn NH: Have tumor suppressor PDCD4 and its countereagent miR-21 gone rogue? Mol Interv 10: 78-79, 2010.

67. St John MA, Dohadwala M, Luo J, Wang G, Lee G, Shih H, Heinrich E, Krysan K, Walser Y, Hazra S, Zhu L, Lai C, Abermayor E, Fishbein M, Elashoff DA, Sharma A and Dubinett SM: Proinflammatory mediators upregulate snail in head and neck squamous cell carcinoma. Clin Cancer Res 15 6018-6027, 2009.

68. Du F, Nakamura Y, Tan TL, Lee P, Lee R, Yu B and Jamora C Expression of snail in epidermal keratinocytes promotes cutaneous inflammation and hyperplasia conducive to tumor formation. Cancer Res 70: 10080-10089, 2010.

69. Albesiano E, Davis M, See AP, Harr JE, Lim M, Pardoll DM and Kim Y: Immunologic consequences of signal transducers and activators of transcription 3 activation in human squamous cell carcinoma. Cancer Res 70: 6467-6476, 2010.

70. Maniati E, Soper R and Hagemann T: Up for mischief? IL-17/ Th17 in the tumour microenvironment. Oncogene 29: 5653-5682, 2010.

71. Cao J, Zhou J, Gao Y, Gu L, Meng H, Liu H and Deng D Methylation of p16 CpG island associated with malignant progression of oral epithelial dysplasia: a progressive cohort study. Clin Cancer Res 15: 5178-5183, 2009.

72. Li L, Zhou J, Gao Y, Gu L, Meng H, Liu H and Deng D: The tumor suppressor UCHL1 forms a complex with p53/MDM2/ ARF to promote 53 signaling and is frequently silenced in nasopharyngeal carcinoma. Clin Cancer Res 16: 2949-2958, 2010.

73. Mohammed J, Ryscavage A, Perez-Lorenzo R, Gunderson AJ, Blazarin N and Glick AB: TGFbeta 1-induced inflammation in premalignant epidermal squamous lesions requires IL-17. J Invest Dermatol 130: 2295-2303, 2010.

74. Yang G and Yang X: Smad4-mediated TGF-beta signaling in tumorigenesis. Int J Biol Sci 6: 1-8, 2010.
75. Wang X, Sun W, Zhang C, Ji G, Ge Y, Xu Y and Zhao Y: TGF- $\beta 1$ inhibits the growth and metastasis of tongue squamous carcinoma cells through Smad4. Gene 485: 160-166, 2011.

76. Meng W, Xia Q, Wu L, Chen S, He X, Zhang L, Gao G and Zhou H: Downregulation of TGF-beta receptor types II and III in oral squamous cell carcinoma and oral carcinoma-associated fibroblasts. BMC Cancer 11: 88, 2011.

77. Shinriki S, Jono H, Ota K, Ueda M, Kudo M, Ota T, Oike Y, Endo M, Ibusuki M, Hiraki A, Nakayamab H, Yoshitakeb Y, Shinohara M and Ando Y: Humanized anti-interleukin-6 receptor antibody suppress tumor angiogenesis and in vivo growth of human squamous cell carcinoma. Clin Cancer Res 15: 5426-5434, 2009.

78. Rao SK, Pavicevic Z, Du Z, Kim JG, Fan M, Jiao Y, Rosenbush M, Samant S, Gu W, Pfeffer LM and Nosrat CA: Pro-inflammatory genes as biomarkers and therapeutic targets in oral squamous cell carcinoma. J Biol Chem 285: 32512-32521, 2010.

79. Wen X-Z, Akiyama Y, Pan K-F, Liu Z-J, Lu Z-M, Zhou J, Gu L-K, Dong C-X, Zhu B-D, Ji J-F, You W-C and Deng D-J: Methylation of GATA-4 and GATA-5 and development of sporadic gastric carcinomas. World J Gastroenterol 16: 1201-1208, 2010.

80. Gasche JA, Hoffman J, Boland CR and Goel A: Interleukin-6 promotes tumorigenesis by altering DNA methylation in oral cancer cells. Int J Cancer 129: 1053-1063, 2011

81. Zhu GQ, Tang YL, Zheng M, Jiang J, Li XY, Chen SX and Liang XH: Hypoxia inducible factor $1 \alpha$ and hypoxia inducible factor $2 \alpha$ play distinct and functionally overlapping roles in oral squamous cell carcinoma. Clin Cancer Res 16: 4732-4741, 2010.

82. Singh B, Stoffel A, Gogineni S, Poluri A, Pfister DG, Shaha AR, Pathak A, Bosl G, Cordon-Cardo C, Shah JP and Rao PH: Amplification of the 3q26.3 locus is associated with progression to invasive cancer and is a negative prognostic factor in head and neck squamous cell carcinomas. Am J Pathol 161: 365-371, 2002.

83. Kikkawa N, Hanazawa T, Fujimura I, Nohata N, Suzuki H, Chazono H, Sakurai D, Horiquchi S, Okamoto Y and Seki N: miR-489 is a tumour-suppressive miRNA target PTNP11 in hypopharyngeal squamous cell carcinomas (HSCC). Br J Cancer 103: 877-884, 2010.

84. Zhang J and Sarge KD: Identification of a polymorphism in the RING finger of human Bmi-1 that causes its degradation by the ubiquitin-proteasome system. FEBS Lett 583: 980-984, 2009.

85. Lo WL, Yu CC, Chiou GY, Chen YW, Huang PI, Chen CS, Tseng LM, Chiu PY, Lu KH, Chang KW, Kao SY and Ciou SH: MicroRNA-200c attenuates tumour growth and metastasis of presumptive head and neck squamous cell carcinoma stem cells. J Pathol 22: 482-495, 2011.

86. Takeyama Y, Sato M, Horio M, Hase T, Yoshida K, Yokoyama T, Kondo M and Hasedawa Y: Knockdown of ZEB1, a master epithelial-to-mesenchymal transition (EMT) gene, suppresses anchorage-independent cell growth of lung cancer cells. Cancer Lett 296: 216-224, 2010.

87. Gibbs EA, Enfield KS, Tsui IF, Chari R, Lam S, Alvarez CE and Lam WL: Deciphering squamous cell carcinoma using multidimensional genomic approaches. J Skin Cancer 2011: 541405, 2011

88. Lockwood WW, Chari R, Coe BP, Thu KL, Garnis C, Malloff CA, Campbell J, Williams AC, Hwang D, Zhu CQ, Buys TPH, Yee J, English JC, MacAulay C, Tsao MS, Gazdar AF, Minna JD, Lam S and Lam WL: Integrative genomic analyses of BRF2 as a novel lineage-specific oncogene in lung squamous cell carcinoma. PLoS Med 7: e1000315, 2010.

89. Sanduja S, Blanco FF and Dixon DA: The roles of TTP and BRF proteins in regulated mRNA decay. Wiley Interdiscip Rev RNA 2: 42-57, 2010

90. Rorke EA, Adhikary G, Jans R, Crish JF and Eckert RL: P1 factor inactivation in the suprabasal epidermis causes increased epidermal hyperproliferation and hyperkeratosis but reduced carcinogendependent tumor formation. Oncogene 29: 5873-5882, 2010.

91. Endo-Munoz L, Dahler A, Teakle N, Rickwood D, HazarRethinam M, Abdul-Jahbar I, Sommerville S, Dickinson I, Kaur PO, Paquet-Fifield S and Saunders N: DE2F7 can regulate proliferation, differentiation, and apoptotic responses in human keratinocytes: implications for cutaneous squamous cell carcinoma formation. Cancer Res 69: 1800-1808, 2009.

92. Carcagno AL, Marazita MC, Ogara MF, Ceruti JM, Sonzogni SV, Scassa ME, Giono LE and Cánepa ET: E2F1-mediated upregulation of p19INK4d determines its periodic expression during cell cycle and regulates cellular proliferation. PLoS One 6: e21938, 2011. 
93. Rowland BD, Denissov SG,Douma S, Stunnenberg KG, Bernards R and Peeper DS: E2F transcriptional repressor complexes are critical downstream targets of p19(ARF) p53-induced proliferative arrest. Cancer Cell 2: 55-85, 2002.

94. Jiang L, Liu X, Chen Z, Jin Y, Heidbreder CE, Kolokythas A, Wang A, Dai Y and Zhou X: MicroRNA-7 targets IGF1R (insulin-like growth factor 1 receptor) in tongue squamous cell carcinoma cells. Biochem J 432: 199-205, 2010.

95. Friedrich RE, Hagel C and Bartel-Friedrich S: Insulin-like growth factor-1 receptor (ICF-1R) in primary and metastatic undifferentiated carcinoma of the head and neck: a possible target of immunotherapy. Anticancer Res 30: 1641-1643, 2010.

96. Kong KL, Kwong DL, Chan TH, Law SY, Chen L, Li Y, Qin YR and Guan XY: MicroRNA-375 inhibits tumour growth and metastasis in oesophageal squamous cell carcinoma through repressing insulin-like growth factor 1 receptor. Gut 2011 [Epub ahead of print].

97. Alzon F, Tabermero J, Cervantes A, Prudkin L, Andreu J, Rodriguez-Braun AHJ, Domingo A, Guijarro J, Gamez C, Rodon J, Di Cosimo S, Brown H, Clark J, Hardwick J, Beckman RA, Hanley W, Hsu K, Calvo E, Rosello S, Langdon RB and Baselga J: A phase I, pharmacokinetic and pharmacodynamic study of dalotuzumab (MK-0646), an anti-IGF-1R monoclonal antibody, in patients with advanced solid tumors. Clin Cancer Res 17: 6304-6312, 2011.

98. Galer CE, Corey CL, Wang Z, Younes MN, Gomez-Rivera F, Jasser SA, Ludwig DL, El-Naggar AK, Weber PS and Myers JN: Dual inhibition of epidermal growth factor receptor and insulinlike growth factor receptor I: reduction of angiogenesis and tumor growth in cutaneous squamous cell carcinoma. Head Neck 33: 189-198, 2011.

99. Oksenych V, Bernardes de Jesus B, Zhovmer A, Egly J-M and Coin F: Molecular insight into the recruitment of TFIIH to sites of DNA damage. EMBO J 28: 2971-2980, 2009.

100. Oksenych V and Coin F: The long unwinding road: XPB and XPD helicases in damaged DNA opening. Cell Cycle 9: 90-96, 2010

101. Fuss JG and Tainer JA: XPB and XPB helicases in TFIIH orchestrate DNA duplex opening and damage verification to coordinate repair with transcription and cell cycle via CAK kinase. DNA Repair (Amst) 10: 697-713, 2011.

102. Egly JM and Coin F: A history of TFIIH: two decades of molecular biology on a pivotal transcription/repair factor. DNA Repair (Amst) 10: 714-721, 2011

103. Lovatt T, Allderea J, Lear JT, Hoban PR, Ramachandran S, Fryer AA, Smith AG and Strange RC: Polymorphism in the nuclear excision repair gene ERCC2/XPD: association between an exon 6-exon 10 haplotype and susceptibility to cutaneous basal cell carcinoma. Hum Mutat 25: 353-359, 2005 .

104. Caro I and Low JA: The role of hedgehog signaling pathway in the development of basal cell carcinoma and opportunities for treatment. Clin Cancer Res 16: 3335-3339, 2010.

105. Von Hoff DD, Lo Russo PM, Rudin CM, Reddy JC, Yauch RL, Tibed R, Weiss GJ,Borad MJ,Hann CL, BrahmerJR, Mackey HM, Lum BK, Darbonne WC, Marsters JC Jr, De Sauvage FJ and Low JA: Inhibition of the hedgehog pathway in advanced basalcell carcinoma. N Engl J Med 361: 1164-1172, 2009.

106. Lofti R, Lere JL and Lotze MT: Eosinophil granulocytes and damage-associated molecular pattern molecules (DAMPS). J Immunother 30: 16-28, 2007.

107. Van Deventer HW, Burgents JE, Wu QP, Woodford RM, Brickey WJ, Allen IC, McElvania-Tekippe E, Serodt JS and Ting JP: The inflammasome component NLRP3 impairs antitumor vaccine by enhancing the accumulation of tumorassociated myeloid-derived suppressor cells. Cancer Res 70 10161-10169, 2010.

108. Sinkovics JG: Antileukemia and antitumor effects of the graftversus-host disease: a new immunovirological approach. Acta Microbiol Immunol Hung 57: 253-347, 2010.

109. Lamkanfi M and Dixit VM: Modulation of inflammasome pathways by bacterial and viral pathogens. J Immunol 187: 597-602 2011.

110. De Nardo D and Latz E: NLRP3 inflammasomes link inflammation and metabolic disease. Trends Immunol 32: 373-379, 2011.

111. Skeldon A and Saleh M: The inflammasomes: molecular effector of host resistance against bacterial, viral, and fungal infections. Front Microbiol 2: 15, 2011

112. Amer AIO: The inflammasome. Front Microbiol 2: 4, 2011.
113. Anand PK, Malireddi RK and Kanneganti TD: Role of the Nlrp3 inflammasome in microbial infection. Front Microbiol 2: 12 , 2011.

114. Negroni G: The role of mycoplasmas and viruses in human leukemia. Proc R Soc Med 59: 662-663, 1966.

115. Fallon RJ, Grist NR, Inman DR, Lemcke RM, Negroni G and Woods DA: Further studies of agents isolated from tissue cultures inoculated with human leukemia bone marrow. Br Med J 14: 388-391, 1965.

116. Murphy WH, Ertel IJ and Zarafonetis CJ: Mycoplasma studies of human leukemia. Ann NY Acad Sci 143: 544-556, 1967.

117. Sinkovics J: Die Grundlagen der Virusforschung. Verlag der Ungarischen Akademie der Wissenschaften, Budapest, pp420, 1956.

118. Sinkovics J: Pleuropneumonie-ähnliche eigenheiten in alten E. coli kulturen. Acta Microbiol Hung 4: 59-75, 1957.

119. Lederberg J and St Clair J: Protoplasts and L-type growth of Escherichia coli. J Bacteriol 75: 143-160, 1958.

120. Sabin B: Nature and source of mycoplasma in various tissue cultures. Ann NY Acad Sci 143: 628-634, 1967.

121. Dmochowski L, Dreyer DA, Grey CE, Hales R, Langford PL, Pipes F, Recher L, Seaman G, Shively JA, Shullenberger CC, Sinkovics JG, Taylor HG, Tessmer CF and Yumoto T: Studies on the submicroscopic morphology of structures resembling mycoplasma and virus particles in mice and men. Ann NY Acad Sci 143: 578-607, 1967.

122.Lo SC, Shih JW, Newton PB III, Wong DM, Hayes MM, Benish JR, Wear DJ and Wang RY: Virus-like infectious agent (VLIA) is a novel pathogenic mycoplasma: Mycoplasma incognitus. Am J Trop Med Hyg 41: 586-600, 1989.

123. Hu WS, Hayes MM, Wang RY, Shih JW and Lo SC: High frequency DNA rearrangement in the chromosomes of clinically isolated Mycoplasma fermentans. Curr Microbiol 37: 1-5, 1998.

124. Sasaki T, Sasaki Y, Kita M, Suzuki K, Watanabe H and Honda M: Evidence that Lo's mycoplasma (Mycoplasma fermentans incognitus) is not a unique strain among Mycoplasma fermentans strains. J Clin Microbiol 30: 24536-24540, 1992.

125. Deffenbacher KE, Iqbal J, Liu K and Chan WC: Recurrent chromosomal alterations in molecularly classified AIDS-related lymphomas; an integrated analysis of DNA copy number and gene expression. J Acquir Immune Defic Syndr 54: 18-26, 2010.

126. Huang XS, Li JY, Wu J, Meng Land Shou CC: Mycoplasma infections and different human carcinomas. World J Gastroenterol 7: 266-269, 2001

127. Chan PJ, Serai IM, Kaloudan TH and King A: Prevalence of mycoplasma conserved DNA in malignant ovarian cancer detected using sensitive PCR-ELISA. Gynecol Oncol 63: 258-260, 1996.

128. Tsai S, Wear DJ, Shih JW and Lo SC: Mycoplasmas and oncogenesis: persistent infection and multistage malignant transformation. Proc Natl Acad Sci USA 92: 10197-10201, 1995.

129. Zhang B, Tsai S, Shih JW, Wear DJ and Lo SC: Absence of mycoplasmal gene in malignant mammalian cells transformed by chronic persistent infection of mycoplasma. Proc Soc Exp Biol Med 218: 83-89, 1998

130. Zhang S, Tsai S and Lo SC: Alteration of gene expression profile during mycoplasma-induced malignant cell transformation. BMC Cancer 6: 116, 2006.

131. Katzav S, Martin-Zanca D and Barbacid M: vav, a novel human oncogene derived from a locus ubiquitously expressed in hematopoietic cells. EMBO J 8: 2283-2290, 1989.

132. Coppola J, Bryant S, Koda T, Conway D and Barbacid M Mechanism of activation of the vav protooncogene. Cell Growth Differ 2: 95-105, 1991.

133. Couceiro JR, Martin-Bermudo MD and Bustelo X: Phylogenetic conservation of the regulatory and functional properties of the Vav oncoprotein family. Exp Cell Res 308: 364-380, 2005.

134. Malartre M, Avaz D, Amador FF and Martin-Bermudo MD The guanine exchange factor vav controls axon growth and guidance during Drosophila development. J Neurosci 30: 2257-2267, 2010.

135. Afshari FT, Kwok JC and Fawcett JW: Astrocyte-produced ephrins inhibit Schwann cell migration via VAV2 signaling. J Neurosci 30: 4246-4255, 2010.

136. Hoffmann A, Aloyz R, Baker K, Dienhofer S, Owens T, Sladek R and Tzankov A: Vav-1 expression correlates with NFKB activation and CD40-mediated cell death in diffuse large B-cell lymphoma cell lines. Hematol Oncol 28: 142-150, 2010. 
137. Martinerie C, Cannizzaro LA, Croce CM, Huebner K, Katzav S and Barbacid M: The human VAV proto-oncogene maps to chromosome region 19p12-19.p13.2. Hum Genet 86: 65-68, 1990.

138. Dong Z, Liu Y, Levin L, Oleksowicz L, Wang J and Lu S: Vav3 oncogene is involved in regulation of secretory phospholipase A2-IIa expression in prostate cancer. Oncol Rep 25: 1511-1518, 2011

139. Lazer G, Idelchuk Y, Schapira V, Pikarsky E and Katzav S: The hematopoietic specific signal transducer Vavl is aberrantly expressed in lung cancer and plays a role in tumorigenesis. J Pathol 219: 25-34, 2009.

140. Syrjänen K, Mäntyärvi R, Väyrynan M, Castrén O, Yliskoski M and Saarikoski S: Chlamydial cervicitis in women followed-up for human papillomavirus (HPV) lesions of the uterine cervix. Acta Obstet Gynecol Scand 64: 467-471, 1985.

141. Werny DM, Saraiya M, Chen X and Platz EA: Prostate-specific antigen, sexual behavior, and sexually transmitted infections in US men 40-59 years old. 2001-2004 a cross sectional study. Infect Agent Cancer 2: 19, 2007.

142. Stefanovic A and Lossos IS: Extranodal marginal zone lymphoma of the ocular adnexa. Blood 114: 501-510, 2009.

143. Ponzoni M, Dolcetti R, Doglioni C and Ferreri AJM: Bugs and marginal zone lymphoma of the ocular adnexae: is the future already here? Blood 114: 3499, 2009.

144. Stefanovic A and Lossos IS: Variability in the prevalence of Chlamydophila psittaci infection in patients with OAML. Blood 114: 3499-3500, 2009.

145. Ferreri AJ, Dolcetti R, Magnino S, Doglioni C and Ponzoni M: Chlamydial infection: the link with ocular adnexal lymphoma Nat Rev Clin Oncol 6: 658-669, 2009. Erratum 6: 678, 2009.

146. McKelvie PA: Ocular adnexal lymphoma: a review. Adv Anat Pathol 17: 251-261, 2010.

147. Ponzoni M, Bonetti F, Poliani PL, Vermi W, Botteli C, Dolcetti R, Cangi MG, Ferreri AJ, Cin ED, Pasini E, Liserre R, Doglioni C, Rossi G and Facchetti F: Central nervous system marginal zone B-cell lymphoma associated with chlamydophila psittaci infection. Hum Pathol 42: 736-742, 2011.

148. Ferreri AJ, Ponzoni M, Guidoboni M, De Conciliis C, Resti AG, Mazzi B, Lettini AA, Demeter J, Dell'Oro S, Doglioni C, Villa E, Boiocchi M and Dolcetti R: Regression of ocular adnexal lymphoma after Chlamydia psittaci-eradicating antibiotic therapy. J Clin Oncol 23: 5067-5073, 2005.

149. Aigelsreiter A, Gariza T, Deutsch ASJ, Leitner E, BehamSchmid C, Beham A, Popper H, Borel N, Pospischil A, Raderer M Kessier HH and Neumeister P: Chlamydia psittaci infection in nongastrointestinal extranodal MALT lymphomas and their precursor lesions. Am J Clin Pathol 1235: 70-75, 2011.

150. Di Felice D, David S, Cappello F, Farina F and Zummo G: Is chlamydial heat shock protein 60 a risk factor for oncogenesis? Cell Mol Life Sci 62: 4-9, 2005.

151. Dolcetti R, Ponzoni M, Ferreri AJ and Godlioni C: Genetic and epigenetic changes linked to Chlamydophila psittaci-associated ocular adnexal lymphomas. Hematol Oncol 28: 1-2, 2010.

152. Sinkovics JG, Trujillo JM, Pienta RJ and Ahearn MJ: Leukemogenesis stemming from autoimmune disease. In: Genetic Concepts and Neoplasia. The University of Texas M.D. Anderson Hosptal, Williams \& Wilkins, Baltimore, MD, pp138-190, 1970

153. Carugi A, Onnis A, Antonicelli G, Rossi B, Mannucci S, Luzzi A, Lazzi S, Bellan C, Tosi GM, Sayed S, De Falco G and Leoncini L: Geographic variation and environmental conditions as cofactors in Chlamydia psittaci association with ocular adnexal lymphomas a comparison between Italian and African samples. Hemato Oncol 28: 20-26, 2010

154. Shinohara H, Yagita H, Ikawa Y and Oyaizu N: Fas drives cell cycle progression in glioma cells via extracellular signalregulated kinase activation. Cancer Res 60: 1766-1771, 2000.

155. Gorina R, Font-Nieves M, Márquez-Kisinousky L, Santalucia T and Planas AM: Astrocyte TLR4 activation induces a proinflammatory environment through the interplay between MyD88-dependent NFкB signaling, MAPK, and Jak1/Stat1 pathways. Glia 59: 242-255, 2011

156. Tafani M, Di Vito M, Frati A, Pellegrini L, De Santis E, Sette G, Eramo A, Sale P, Mari E, Santoro A, Raco A, Salvati M, De Maria R and Russo MA: Pro-inflammatory gene expression in solid glioblastoma microenvironment and in hypoxic stem cells from human glioblastoma. J Neuroinflammation 8: 32, 2011.

157. Synowitz M, Glass R, Färber K, Markovic D, Kronenberg G, Herrmann K, Schnermann J, Nolte C, van Rooijen N and Kettenmann H: A1 adenosine receptor in microglia control glioblastoma-host interactions. Cancer Res 66: 8550-8557, 2006.
158. Alves TR, Lima FR, Kahn SA, Lobo D, Dubois LG, Soletti R, Borges H and Neto VM: Glioblastoma cells: a heterogeneous and fatal tumor interacting with the parenchyma. Life Sci 89: 532-539, 2011

159. Charles NA, Hollad EC, Gilbertson R, Glass R and Kettenmann H: The brain tumor microenvironment. Glia 59: 1169-1180, 2011.

160. Wesolowska A, Kwiatowska A, Slomnicki L, Dembinski M, Master A, Sliwa M,Franciszkiewicz K, Chouaib S and Kaminska B Microglia-derived TGF-beta as an important regulator of glioblastoma invasion - an inhibition of TGF-beta-dependent effects by shRNA against human TGF-beta type II receptor. Oncogene 27: 918-930, 2008

161. Sinkovics JG and Horvath JC: The molecular biology and immunology of glioblastoma multiforme (GBM) with the presentation of an immunotherapy protocol for a clinical trial. Acta Microbiol Immunol Hung 53: 367-429, 2006.

162. Heimberger AB, Crotty LE, Archer GE, McLendon RE, Friedman A, Dranoff G, Bigner DD and Sampson JH: Bone marrow-derived dendritic cells pulsed with tumor homogenate induce immunity against syngeneic intracerebral glioma. J Neuroimmunol 103: 16-25, 2000.

163. Mineharu Y,King GD, Muhammad AG, Bannykh S, Kroeger KM, Liu C, Lowenstein PR and Castro MG: Engineering the brain tumor microenvironment enhances the efficacy of dendritic cell vaccination: implications for clinical trial. Clin Cancer Res 17: 4705-4718, 2011.

164. Sinkovics JG and Horvath JC: Natural and genetically engineered viral agents for oncolysis and gene therapy of human cancers. Arch Immunol Ther Exp 56: 30S-59S, 2008.

165. Calzascia T, Di Bernardino-Besson W, Wimotte R, Masson F, De Tribolet N, Dietrich PY and Walker PR: Cutting edge: cross presentation mechanism for efficient recruitment of tumor-specific CTL to the brain. J Immunol 171: 2187-2191, 2003.

166. Iwadate Y, Yamaura A, Sakiyama S, Sato Y and Tagawa M Glioma-specific cytotoxic T cells can be effectively induced by subcutaneous vaccination of irradiated wild-type tumor cells without artificial cytokine production. Int J Oncol 23: 483-488, 2003.

167. Vauleon E, Avril T, Collet B, Mosser J and Quillien V: Overview of cellular immunotherapy for patients with glioblastoma. Clin Dev Immunol pii: 689171, 2010.

168. Dillman RO, Duma CM, Ellis RA, Cornforth AN, Schlitz PM, Sharp SL and De Priest MC: Intralesional lymphokine-activated killer cells as adjuvant therapy for pimary glioblastoma. J Immunother 32: 914-919, 2009.

169. Castriconi R, Daga A, Dondero A,Zona G, Poliani PL, Melotti A, Griffero F, Marubbi D, Spaziante R, Bellora F, Moretta L, Moretta A, Corte G and Bottino C: NK cells recognize and kill human glioblastoma cells with stem cell-like properties. J Immunol 182: 353-359, 2009.

170. Crane CA, Han SJ, Barry JJ, Ahn BJ, Lanier LL and Parsa AT: TGF-beta downregulates the activating receptor NKG2D on NK cells and $\mathrm{CD}^{+} \mathrm{T}$ cells in glioma patients. NeuroOncol 12: 7-13, 2010.

171. Sampson JH, Aldape KD, Archer GE, Coan A, Desjardins A, Friedma AH, Ftriedman HS, Gilbert MR, Herndon JE, McLendon RE, Mitchell DA, Reardon DA, Sawaya R, Schmtting R, Shi W, Vredenburgh JJ, Bigner DD and Heimberger AB: Greater chemotherapy-induced lymphopenia enhances tumorspecific immune responses that eliminate EGFRvIII-expressing tumor cells in patients with glioblastoma. NeuroOncol 13: 324-333, 2011

172. Sampson JH, Heimberger AB, Archer GE, Aklape KD Friedman AH, Friedman HS, Gilbert MR, Herndon JE II, McLendon RE, Mitchell DA, Reaerdon DA, Sawaya R, Schmittling RJ, Shi W, Vredenburgh JJ and Bigner DD: Immunologic escape after prolonged progression-free survival with epidermal growth factor receptor variant III peptide vaccination in patients with newly diagnosed glioblastoma. J Clin Oncol 28: 4722-4729, 2010

173. Kadowaki M, Yoshioka H, Kamitani H, Watanabe T, Wade PA and Eling TE: DNA methylation-mediated silencing of nonsteroidal anti-inflammatory drug-activated gene (NAG-1/ GDF15) in glioma cells. Int J Cancer doi: 10.1002/ijc.26082, 2011.

174. Wang C, Wang $\mathrm{J}$ and Bai P: Troglitazone induces apoptosis in gastric cancer cells through the NAG-1 pathway. Mol Med Report 4: 93-97, 2011 
175. Bokobza SM, Ye L, Kynaston H and Jiang WD: Growth and differentiation factor 9 (GDF-9) induces epithelial-mesenchymal transition in prostate cancer cells. Mol Cell Biochem 349: 33-49, 2010.

176. Liu K-W, Feng H, Bachoo R, Kazlauskas A, Smith EM, Symes K, Hamilton RL, Nagana M, Nishikawa R, Bo H and Cheng S-Y: 2/PTPN11 mediates gliomagenesis driven by PDGFRA and INK4A/ARF aberrations in mice and humans. J Clin Invest 121 905-917, 2011

177. Dong H, Luo L, Hong S, Siu H, Xiao Y, Jin L, Chen R and Xiong $\mathrm{M}$ : Integrated analysis of mutations, miRNA and mRNA expressions in glioblastoma. BMC Syst Biol 4: 163, 2010.

178. Sinkovics JG: The adaptive immune system, autoimmunity and lymphomagenesis. In: Cytolytic Immune Lymphocytes. Schenk Buchverlag, Budapest and Passau, pp43-63, 137-148, 2008.

179. Tiemann M, Asbeck R and Wacker HH: Clonal B cell reaction in Sjögren disease and Hashimoto autoimmune thyroiditis. Pathologe 127: 289-295, 1996.

180. Donckler JE, Michel L, Delos M, Havaux X and van Beneden RL: Interrelated overexpression of endothelial and inducible nitric oxide synthases, endothelin-1 and angiogenic factors in human papillary thyroid carcinoma. Clin Endocrinol 64: 703-710, 2006.

181. Chechlinska M, Kowalewska M and Nowak R: Systemic inflammation as a confounding factor in cancer biomarker discovery and validation. Nat Rev Cancer 10: 2-3, 2010.

182. French JD, Weber ZJ, Fretwell DL, Said S, Klopper JP and Hausen BR: Tumor-associated lymphocytes and increased FoxP $3^{+}$ regulatory $\mathrm{T}$ cell frequency correlate with more aggressive papillary thyroid cancer. J Clin Endocrinol Metab 95: 2325-2333, 2010.

183. Koida S, Tsukasaki K, Tsuchiya T, Harasawa H, Fukushima T, Yamada Y, Ohshima K, Kamihira S, Kikuchi M and Tomonaga M: Primary T-cell lymphoma of the thyroid gland with chemokine receptors of Th1 phenotype complicating autoimmune thyroiditis. Haematologica 92: e37-e40, 2007.

184. Kaczmarek E, Lacka K, Jamolowska-Jurczyszyn D, Sidor A and Majewski P: Changes of B and T lymphocytes and selected apoptosis markers in Hashimoto's thyroiditis. J Clin Pathol 64 626-630, 2011.

185. Saverino D, Brizzolara R, Simone R, Chiappori A, MilinteridaFloriani F, Pesce G and Bagnosco M: Soluble CTLA-4 in autoimmune thyroid diseases: relationship with clinical status and possible role in the immune response dysregulation. Clin Immunol 123: 190-198, 2007.

186. Bozec A, Lassalle S, Hofman Y, Ilie M, Santini J and Hofman P: The thyroid gland: a crossroad in inflammation-induced carcinoma? An ongoing debate with the new therapeutic potential. Curr Med Chem 17: 3449-3461, 2010.

187. Mao C, Wang S, Xiao Y, Xu J, Jiang Q, Jin M, Jiang X, Guo H, Ning $G$ and Zhang Y: Impairment of regulatory capacity of $\mathrm{CD} 4^{+} \mathrm{CD} 25^{+}$regulatory $\mathrm{T}$ cells mediated by dendritic cell polarization and hyperthyroidism in Graves disease. J Immunol 186: 4734-4743, 2011.

188. Orloff M, Peterson C, He X, Ganapahi S, Heald B, Yang YR, Bebek G, Romigh T, Song JH, Wu W, David S, Cheng Y, Meltzer SJ and Eng C: Germline mutations in MSR1, ASCC1, and CTHRCl in patients with Barrett esophagus and esophageal adenocarcinoma. JAMA 306: 410-419, 2011

189. Mao X, Orchard G, Vonderheid EC, Nowell PC, Bagot M, Bensussan A, Russell-Jones R, Young BD and Whittaker SJ: Heterogenous abnormalities of CCND1 and RB1 in primary cutaneous $\mathrm{T}$ cell lymphomas suggesting impaired cell cycle control in disease pathogenesis. J Invest Dermatol 126: 1388-1395, 2006a.

189. Luo KJ, Hu Y and Fu JH: Cyclin D1, p53, E-cadherin, and VEGF discordant expression in paired regional metastatic lymph nodes of esophageal squamous cell carcinoma. A tissue array analysis. J Surg Oncol 104: 236-243, 2011b.

190. Duggan SP, Behan FM, Kirca M, Smith S, Reynolds JV, Long A and Kelleher D: An integrative genomic approach in oesophageal cells identifies TRB3 as a bile acid responsive gene, downregulated in Barrett's oesophagus, which regulates NFkappaB activation and cytokine levels. Carcinogenesis 31: 936-945, 2010.

191. Kiss-Toth E: Tribbles, 'puzzling' regulators of cell signaling. Biochem Soc Trans 39: 684-687, 2011.

192. Zhang HY, Zhang Q, Zhang X, Yu C, Huo X, Cheng E, Wang DH, Spechler SJ and Souza RF: Cancer-related inflammation and Barrett's carcinogenesis: interleukin-6 and STAT3 mediate apoptotic resistance in transformed Barrett's cells. Am J Physiol Gastrointest Liver Physiol 300: G454-G460, 2011.
193. Abdel-Latif MM, Duggan S, Reynolds JV and Kelleher D: Inflammation and esophageal carcinogenesis. Curr Opin Pharmacol 9: 396-404, 2009.

194. Farinati F, Piciocchi M, Lavezzo E, Bortolami M and Cardin R: Oxidative stress and inducible nitric oxide synthase induction in carcinogenesis. Dig Dis 28: 579-584, 2010.

195. Ferguson HR, Wild CP, Aderson LA, Murphy SJ, Johnston BT, Murray LJ, Watson RG, McGuigan J, Reynolds JV and Hardie LJ: Cyclooxygenase- 2 and inducible nitric oxide synthase gene polymorphism and risk of reflux esophagitis, Barrett's esophagus, and esophageal adenocarcinoma. Cancer Epidemiol Biomarkers Prev 17: 727-731, 2008.

196. Konturek PC, Kania J, Burnat G, Hahn EG and Konturek SJ: Prostaglandins as mediators of COX-2 derived carcinogenesis in gastrointestinal tract. J Physiol Pharmacol 56 (Suppl 5): 57-73, 2005.

197. Nguyen GH, Scheter AJ, Chou DB, Bowman ED, Zhao R, Hawkes JE, Mathé EA, Kumamoto K, Zhao Y, Budhu A, Hagiwara N, Wang XW, Miyashita M, Casson AG and Harris CC: Inflammatory and microRNA expression as prognostic classifier of Barrett's associated esophageal adenocarcinoma. Clin Cancer Res 126: 5824-5834, 2010.

198. Zhang HY, Hormi-Carver K, Zhang X, Spechler SJ and Souza RF: In benign Barrett's epithelial cells, acid exposure generated reactive oxygen species that cause DNA double-strand breaks. Cancer Res 69: 9083-9089, 2009.

199. Mathé EA, Nguyen GH, Bowman ED, Zhao Y, Budhu A, Schetter AJ, Braun R, Reimers M, Kumamoto K, Hughes D, Altorki NK, Casson AG, Liu C-G, Wang XW, Croce CM and Harris CC: MicroRNA expression in squamous cell carcinoma and adenocarcinoma of the esophagus: association with survival. Clin Cancer Res 15: 6192-6200, 2009.

200. Miron N, Miron MM, Milea VG and Cristea V: Proinflammatory cytokines: an insight into pancreatic oncogenesis. Roum Arch Microbiol Immunol 69: 183-189, 2010.

201. Achyut BR and Yang L: Transforming growth factor- $\beta$ in the gastrointestinal and hepatic tumor microenvironment. Gastroenterology 141: 1167-1178, 2011

202.Liles JS, Arnoletti JP, Kossenkov AV, Mikhaylina A, Frost AR, Kulesza P, Heslin MJ and Frolov A: Targeting ErbB3-mediated stromal epithelial interactions in pancreatic ductal adenocarcinoma. Br J Cancer 105: 523-533, 2011.

203. Tang SN, Fu J, Nall D, Rodova M, Shankar S and Srivastava RK: Inhibition of sonic hedgehog pathway and pluripotency maintaining factors regulate human pancreatic cancer stem cell characteristics. Int J Cancer doi: 10.1002/ijc.26323, 2011

204. Izeradjene $K$ and Hingorani SR: Targets, trials, and travails in pancreatic cancer. J Natl Compr Cancer Netw 5: 1042-1053, 2007.

205. Fogar P, Basso D, Fadi E, Greco E, Pantano G, Padoan A, Bozzato A, Facco M, Sanzari MC, Teolato S, Zambon CE, Navaglia F, Semenzato G, Pedrazzoli S and Plebani M: Pancreatic cancer alters human $\mathrm{CD}^{+}$lymphocyte function: a piece in the immune evasion puzzle. Pancreas 40: 1131-1137, 2011

206. Martin P and Sanchez-Madrid E: CD69: an unexpected regulator of Th17 cell-driven inflammatory response. Sci Signal 4(165): pe14, 2011 .

207. Hinz S, Pagerols-Raluy L, Oberg HH, Ammerpohl O, Grüssel S, Sipos B, Grützmann R, Piarsky C, Ungerfroren H, Saeger HD, Klöppel G, Kabelitz D and Kalthoff H: Foxp3 expression in pancreatic carcinoma cells as a novel mechanism of immune evasion in cancer. Cancer Res 67: 8344-8350, 2007.

208. Puthenedam M, Wu F, Shetye A, Michaels A, Rhee KJ and Kwon JH: Matrilysin-1 (MMP7) cleaves galectin-3 and inhibits wound healing in intestinal epithelial cells. Inflamm Bowel Dis 17: 260-267, 2011.

209. Fukuda A, Wang SC, Morris JP IV, Folias AE, Liou A, Kim GE, Akira S, Boucher KM, Firpo MA, Mulvihill SJ and Hebrok M: Stat3 and MMP7 contribute to pancreatic ductal adenocarcinoma initiation and progression. Cancer Cell 19: 441-455, 2011.

210. Zhang Y, Bharadwaj U, Logsdon CD, Chen C, Yao Q and Li M: ZIP4 regulates pancreatic cancer cell growth by activating IL-6/ STAT3 pathway through zinc finger transcription factor CREB. Clin Cancer Res 16: 1423-1430, 2010.

211. Li M, Zhang Y, Bharadwaj U, Zhai QJ, Ahern CH, Fisher WE, Brunicardi FC, Logsdon CD, Chen C and Yao Q: Downregulation of ZIP by RNA interference inhibits pancreatic cancer growth and increases the survival of nude mice with pancreatic cancer xenografts. Clin Cancer Res 15: 5993-6001, 2009. 
212. Tan L, Sui X, Deng H and Ding M: Holoclone forming cells from pancreatic cancer cells enrich tumor initiating cells and represent a novel model for study of cancer stem cells. PLoS One 6: e23383, 2001.

213. Li W, Lebrun DG and Li M: The expression and functions of microRNAs in pancreatic adenocarcinoma and hepatocellular carcinoma. Chin J Cancer 30: 540-550, 2011.

214. Wang J and Sen S: MicroRNA functional network in pancreatic cancer: from biology to biomarkers of disease. J Biosci 36 : 481-491, 2011.

215. Hamacher R, Diersch S, Scheibel M, Eckel F, Mayr M, Rad R, Bajbouj M, Schmid RM, Saur D and Schneider G: Interleukin-1 beta gene promoter SNPs are associated with risk of pancreatic cancer. Cytokine 46: 182-186, 2009.

216. Bauer L, Venz S, Junker H, Brandt R and Radons J: Nicotinamide phosphoribosyltransferase and prostaglandin $\mathrm{H} 2$ synthase 2 are up-regulated in human pancreatic adenocarcinoma cells after stimulation with interleukin-1. Int J Oncol 35 97-107, 2009

217. Guo S and Gonzalez-Perez RR: Notch, IL-1 and leptin crosstalk outcome (NILCO) is critical for leptin-induced proliferation, migration and VEGF/VEGFR-2 expression in breast cancer. PLoS One 6: e21467, 2011.

218. Olkhanud PB, Rochman Y, Bodogai M, Malchinkhuu E, Wejksza K, Xu M, Gress RE, Hesdorffer C, Leonard WJ and Biragyn A: Thymic stromal lymphopoietin is a key mediator of breast cancer progression. J Immunol 186: 5656-5662, 2011

219. Sangaletti S, Tripodo C, Ratti C, Piconese S, Porcasi R, Salcedo R, Trinchieri G, Colombo MP and Chiodoni C: Oncogene-driven intrinsic inflammation induces leukocyte production of tumor necrosis factor that critically contributes to mammary carcinogenesis. Cancer Res 70: 7764-7775, 2010.

220. Yang Y, Feng R, Bi S and Xu Y: TNF-alpha polymorphisms and breast cancer. Breast Cancer Res Treat 129: 513-519, 2011.

221. Gong Y, Huo L, Liu P, Sneige N, Sun X, Ueno NT, Lucci A, Buchholz TA, Valero V and Cristofanilli M: Polycomb group protein EZH2 is frequently expressed in inflammatory breast cancer and is predictive of worse clinical outcome. Cancer doi: 10.1002/cncr.26179, 2011.

222. Aird KM, Allensworth JL, Batinic-Haberle I, Lyerly HK, Dewhirst MW and Devi GR: ErbB1/2 tyrosine kinase inhibitor mediates oxidative stress-induced apoptosis in inflammatory breast cancer cells. Breast Cancer Res Treat 2011 [Epub ahead of print].

223. Mahmoud SM, Paish EC, Powe DG, Macmillan RD, Grainge MJ, Lee AH, Ellis IO and Green AR: Tumor-infiltrating CD8 lymphocytes predict clinical outcome in breast cancer. J Clin Oncol 29: 1949-1955, 2011

224. Santisteban M, Reiman JM, Asiedu MK, Behrens MD, Nassar A Kalli KR, Haluska P, Ingle JN, Hartmann LC, Manjili MH, Radisky DC, Ferrone S and Knutson KL: Immune-induced epithelial to mesenchymal transition in vivo generates breast cancer stem cells. Cancer Res 69: 2887-2895, 2009.

225. Sinkovics JG: Horizontal gene transfers and cell fusions in microbiology, immunology and oncology. Int J Oncol 35: 441-465, 2009.

226. Gobert M, Treilleux I, Bendriss-Vermare N, Bachelot T, GoddardLeon S, Arfi V, Biota C, Doffin AC, Durand I, Olive D, Perez S, Pasqual N, Faure C, Ray-Coquard I, Puisieux A, Caux C, Bay J-Y and Ménétrier-Caux C: Regulatory $\mathrm{T}$ cells recruited through CCL22/CCR4 are selectively activated in lymphoid infiltrates surrounding primary breast tumors and lead to an adverse clinical outcome. Cancer Res 69: 2000-2009, 2009a.

226. Pogo BG, Melana SM, Moran H and Holland JF: Presence of MMTV-like env gene sequence in human breast cancer. Breast Cancer Res Treat 125: 295-297, 2011b.

227. Mine T, Matsueda S, Li Y, Tokumitsu H, Gao H, Danes C, Wong KK, Wang X, Ferrone S and Ioannides CG: Breast cancer cells expressing stem cell markers $\mathrm{CD} 44^{+} \mathrm{CD} 24$ low are eliminated by Numb-1 peptide-activated T cells. Cancer Immunol Immunother 58: 1185-1194, 2009.

228. Ding X, Zhu F, Li T, Zhou Q, Hou FF and Nie J: Numb protects renal proximal tubular cells from puromycin aminonucleosideinduced apoptosis through inhibiting Notch signaling pathway. Int J Biol Sci 7: 269-278, 2011.

229. Lee DF, Kuo HP, Chen CT, Hsu JM, Chou CK, Wei Y, Sun HL, Li LY, Ping B, Huang WC, He X, Hung JY, Lai CC, Ding Q, Su JL, Yang JY, Sahin AA, Hortobagyi GN, Tsai FJ, Tsai CH and Hung MC: IKK beta suppression of TSC1 links inflammation and tumor angiogenesis via the mTOR pathway. Cell 130 $440-455,2007$.
230. Lee C, Dhillon J, Wang MY, Gao Y, Hu K, Park E, Astanehe A, Hung MC, Eirew OP, Eaves CJ and Dunn SE: Targeting YB-1 in HER-2 overexpressing breast cancer cells induces apoptosis via the mTOR/STAT pathway and suppresses tumor growth in mice. Cancer Res 68: 8661-8666, 2008.

231. Iwamoto T, Bianchini G, Qi Y, Cristofanilli M, Lucci A, Woodward WA, Reuben JM, Matsuoka J, Gong Y,Krishnamurthy S, Valero V, Hortobagyi GN, Robertson F, Symmans WF, Pusztai L and Ueno NT: Different gene expressions are associated with the different molecular subtypes of inflammatory breast cancer. Breast Cancer Res Treat 125: 785-795, 2011.

232. Houchens NW and Merajkver SD: Molecular determinants of the inflammatory breast cancer phenotype. Oncology 27: 1556-1566, 2008.

233. Charafe-Jauffret E, Ginestier C, Iovino F, Tarpin C, Diebel M, Esterni B, Houvenaeghei G, Extra J-M, Bertucci F, Jaquemier J, Xern L, Dontu G, Stassi G, Xiao Y, Barsky SH, Birnbaum D, Viens P and Wicha MS: Aldehyde dehydrogenase 1-positive cancer stem cells mediate metastasis and poor clinical outcome in inflammatory breast cancer. Clin Cancer Res 16: 45-55, 2010.

234. Sun JG, Liao RX, Qiu J, Jin JY, Wang XX, Duan YZ, Chen FL, Hao P, Xie QC, Wang ZX, Li DZ, Chen ZT and Zhang SX: Microarray-based analysis of microRNA expression in breast cancer stem cells. J Exp Clin Cancer Res 29: 174, 2010.

235. Van der Auwera I, Limame R, van Dam P, Vermeulen PB, Dirix LY and van Laere SJ: Integrated miRNA and mRNA expression profiling of the inflammatory breast cancer subtype. Br J Cancer 103: 532-541, 2010.

236. Jiang S, Zhang HW, Lu MH, He XH, Li Y, Gu H, Liu MF and Wang ED: MicroRNA-155 functions as an OncomiR in breast cancer by targeting the suppressor of cytokine signaling 1 gene. Cancer Res 70: 3119-3127, 2010.

237. Glover JA, Hughes CM, Cantwell MM and Murray LJ: A systematic review to establish the frequency of cyclooxygenase-2 expression in normal breast epithelium, ductal carcinoma in situ, microinvasive carcinoma of the breast and invasive breast cancer. Br J Cancer 105: 13-17, 2011

238. Yi Lau GT and Leung LK: The dietary flavonoid apigenin blocks phorbol 12-myristate 13-acetate-induced COX-2 transcriptional activity in breast cell line. Food Chem Toxicol 48: 3022-3027, 2010.

239. De Jong D, Vasmel WLE, De Boer JP, Vrhave G, Barbé E, Casparie MK and van Leewen FE: Anaplastic large cell lymphoma in women with breast implants. JAMA 300: 2030-2035, 2008.

240.Lechner MG, Lade S, Liebertz DJ, Prince HM, Brody GS, Webster HR and Epstein AL: Breast implant-associated, ALKnegative, T-cell, anaplastic, large cell lymphoma: establishment and characterization of a model cell line (TLBR-1) for this newly emerging clinical entity. Cancer 117: 1478-1489, 2011

241. Li S and Lee AK: Silicone implant and primary breast ALK1negative anaplastic large cell lymphoma, fact or fiction? Int $\mathrm{J}$ Clin Exp Pathol 3: 117-127, 2010.

242. Desjobert C, Renalier MH, Bergalet J, Dejean E, Joseph N, Kruczynski A, Soulier J, Espinos E, Meggetto F, Cavaillé J, Delsol G and Lamant L: MiR-29a down-regulation in ALKpositive anaplastic large cell lymphomas contributes to apoptosis blockade through MCL-1 overexpression. Blood 117: 6627-6637, 2011.

243. Singh RR, Cho-Vega JH, Davuluri Y, Ma S, Kasbidi F, Milito C, Lennon PA, Drakos E, Medeiros LJ, Luthra R and Vega F: Sonic hedgehog signaling pathway is activated in ALK-positive anaplastic large cell lymphoma. Cancer Res 69: 2350-2358, 2009.

244. Vogelstein B, Fearon ER, Hamilton SR, Kern SE, Reisinger AC, Leppert M, Nakamura Y, White R, Smits AM and Bos JL: Genetic alterations during colorectal-tumor development. N Engl J Med 319: 525-532, 1988.

245. Baker SJ, Markowitz S, Fearon ER, Willson KK and Vogelstein B: Suppression of human colorectal carcinoma cell growth by wild-type p53. Science 249: 912-915, 1990.

246. Fearon ER and Vogelstein B: A genetic model for colorectal tumorigenesis. Cell 61: 759-767, 1990.

247. Polyak K, Hamilton SR, Vogelstein B and Kinzler KW: Early alteration of cell-cycle-regulated gene expression in colorectal neoplasia. Am J Pathol 149: 381-387, 1996.

248. Fukata M, Chen A, Vamadevan AS, Cohen J, Bregli K, Krishnareddy S, Xu R,Harpaz N, Dannenberg A,Subbaramaiah K, Cooper HS, Itzkowitz SH and Abreu MT: Toll-like receptor-4 (TLR4) promotes the development of colitis-associated colorectal tumors. Gastroenterology 133: 1869-1881, 2007.

249. Colombo MP and Piconese S: Polyps wrap mast cells and Treg within tumorigenic tentacles. Cancer Res 69: 5619-5622, 2009. 
250. Gounaris E, Blatner NR, Dennis K, Magnusson F, Gurish MF, Strom TB, Beckhove P, Gounari F and Khazale K: T-regulatory cells shift from a protective anti-inflammatory to a cancerpromoting proinflammatory phenotype in polyposis. Cancer Res 69: 490-497, 2009.

251. Salcedo R, Worschech A, Cardone M, Jones Y, Gyulai Z, Dai RM, Wang E, Ma W, Haines D, O'hUigin C, Marincola FM and Trinchieri G: MyD88-mediated signaling prevents development of adenocarcinomas of the colon: role of interleukin-18 J Exp Med 207: 1625-1636, 2010.

252. McLean MH, Murray GI, Stewart KN, Norrie G, Mayer C Hold GL, Thomson J, Fyfe N, Hope M, Mowat NA, Drew JE and El-Omar EM: The inflammatory microenvironment in colorectal neoplasia. PLoS One 6: e15366, 2011.

253. Luley K, Noack F, Lehnert H and Homann N: Local calprotectin production in colorectal cancer and polyps - active neutrophil recruitment in carcinogenesis. Int J Colorectal Dis 26: 603-607, 2011.

254. Hanahan D and Weinberg RA: Hallmarks of cancer: the next generation. Cell 144: 646-674, 2011.

255. Colotta F, Allavena F, Sica A, Garlanda C and Mantovani A: Cancer-related inflammation, the seventh hallmark of cancer: links to genetic instability. Carcinogenesis 30: 1073-1081, 2009

256. Cavallo F, De Giovanni C, Nanni F, Forni G and Lollini PL: 2011: the immune hallmarks of cancer. Cancer Immunol Immunother 60: 319-326, 2011

257. Sipos F and Múzes G: Isolated lymphoid follicles in colon: switch points between inflammation and colorectal cancer? World J Gastroenterol 17: 1666-1673, 2011.

258. Sinkovics J: Stem cells in colonic epithelium (in Hungarian). Orv Hetil (Budapest) 151: 911-912, 2010.

259. Zeki SS, Graham TA and Wright NA: Stem cells and their implications for colorectal cancer. Nat Rev Gastroenterol Hepatol 8: 90-100, 2011

260. Takahashi H, Ishii H, Nishida N, Takemasa I, Mizushima T, Ikeda M, Yokobori T, Mimori K, Yamamoto H, Sekimoto M, Doki Y and Mori M: Significance of Lgt5(+ve) cancer stem cells in the colon and rectum. Ann Surg Oncol 18: 1166-1174, 2011.

261. Tiwari R, Bargmann W and Bose HR Jr: Activation of the TGF- $\beta$ / Smad signaling pathway in oncogenic transformation by v-Rel. Virology 413: 60-71, 2011.

262. Sakamoto K, Maeda S, Hikiba Y, Nakagawa H, Hayakawa Y, Shuibata W, Yanai A, Ogura K and Omara M: Constitutive NFאB activation in colorectal carcinoma plays a key role in angiogenesis promoting tumor growth. Clin Cancer Res 15: 2248-2258, 2009.

263. Deng G, Kakar S, Okudiara K, Choi E, Sleisenger MH and Kim YS: Unique methylation pattern of oncostatin $\mathrm{m}$ receptor gene in cancers of colorectum and other digestive organs. Clin Cancer Res 15: 1519-1526, 2009.

264. Balaguer F, Link A, Lozano JJ, Cuatrecasas M, Boland CR and Goel A: Epigenetic silencing of miR-137 is an early event in colorectal carcinogenesis. Cancer Res 70: 6609-6618, 2010.

265. Schetter AJ, Nguyen GH, Boweman ED, Mathé EA, Yuen ST, Hawkes JE, Croce CM, Leung SY and Harris CC: Association of inflammation-related and microRNA gene expression with cancer specific mortality of colon adenocarcinoma. Clin Cancer Res 15: 5878-5887, 2009

266. Hahn MA, Hahn T, Lee DH, Esworthy RS, Kim BW, Riggs AD, Chu FF and Pfeifer GP: Methylation of polycomb target genes in intestinal cancer is mediated by inflammation. Cancer Res 68: 10280-10289, 2008

267. Wilson AJ, Chueh AC, Tögel L, Comer GA, Ahmed N, Goel S, Byun DS, Nasser S, Houston MA, Jhawer M, Smartt HJ, Murray LB, Nicholas C, Heerdt BG, Arango D, Augenlicht LH and Mariadason JM: Apoptotic sensitivity of colon cancer cells in histone deacetylase inhibitors is mediated by an $\mathrm{Sp} 1 / \mathrm{Sp} 3$ activated transcriptional program involving immediate early gene induction. Cancer Res 70: 609-620, 2010.

268. Tu SP, Sun TW, Cui JT, Zou B, Lin MC, Gu Q, Jiang SH, Kung HF Kornaluk RG and Wong BC: Tumor suppressor XIAP-associated factor 1 (Xiaf) cooperates with tumor necrosis factor-related apoptosis-inducing ligand to suppress colon cancer growth and trigger tumor regression. Cancer 116: 1252-1263, 2010.

269. Nosho K, Shima K, Kure S, Irahara N, Baba Y, Chen L, Kirkner GJ, Fuchs CS and Ogino S: JC virus T-antigen in colorectal cancer is associated with p53 expression and chromosomal instability, independent $\mathrm{CpG}$ island methylator phenotype. Neoplasia 11: $87-95,2009$

270. Sears CL and Pardoll DM: Perspective: alpha bugs, their microbial partners the link to colon cancer. J Infect Dis 203: 306-311, 2011.
271. Ogino S, Nosho K, Irahara N, Meyerhardt JA, Baba Y, Shima K, Glickman JN, Ferrone CR, Mino-Knudson M, Tanaka N, Dranoff G, Giovannucci EL and Fuchs CS: Lymphocyte reaction to colorectal cancer is associated with longer survival, independent of lymph node count, microsatellite instability, and $\mathrm{CpG}$ island methylation phenotype. Clin Cancer Res 15: 6412-6420, 2009.

272. Rosen MJ, Frey MR, Washington MK, Chaturvedi R, Kuhnhein LA, Matta P, Revetta FL, Wilson KT and Polk DB: STAT6 activation in ulcerative colitis: a new target for prevention of IL-13-induced colon epithelial cell dysfunction. Inflamm Bowel Dis doi: 10.1002/ ibd.21628, 2011

273. Saito S, Kato J, Hitraoka S, Horii J, Suzuki H, Higashi R, Kaji E, Kondo $\mathrm{Y}$ and Yamamoto K: DNA methylation of colon mucosa in ulcerative colitis patients: correlation with inflammatory status. Inflamm Bowel Dis 17: 1955-1965, 2011.

274. Czakó L, Gyökeres T, Topa L, Sahin T, Vincze A, Dubravcsik Z Szepes A, Pap A, Földes I, Terzin V, Tiszlavicz L and Wittmann T: Autoimmune pancreatitis in Hungary: a multicenter nationwide study. Pancreatology 11: 261-267, 2011.

275. Ghimire P, Wu G-Y and Zhu L: Primary gastrointestinal lymphoma. World J Gastroenterol 17: 697-707, 2001.

276. Argueso JL, Kijas AW, Sarin S, Heck J, Waase M and Alani E: Systematic mutagenesis of the Saccharomyces cerevisiae MLH1 gene reveals distinct roles for Mlhlp in meiotic crossing over and in vegetative and meiotic mismatch repair. Mol Cell Biol 23: 873-886, 2003.

277. Bonadona V, Bonaïti B, Olschwang S, Grandjouan S, Huiart L, Longy M, Guimband B, Buecher B, Bignon Y-J, Caron O, Colas C, Noguès $C$, Lejeune-Dumoulin $S$, Olivier-Faivre L, PolycarpOsaer F, Nguyen TDF, Deseigne F, Saurin J-C, Berthet P, Leroux D, Duffour J, Manouvrier S, Frébourg T, Sobol H, Lasset $C$ and Bonaïti-Pellié $C$, for the French Cancer Genetics Network: Cancer risks associated with germline mutations in MLH1, MSH2, and MSH6 genes in Lynch syndrome. JAMA 305: 2304-2310, 2011.

278. Lynch HT, Lynch JF, Snyder CL and Riegert-Johnson D: EPCAM deletions, Lynch syndrome, and cancer risk. Lancet Oncol 12: 5-6, 2011

279. Perez-Cabornero L, Infante M, Velasco EA, Lastra E, Acedo A, Miner C and Duran M: Frequency of rearrangements in Lynch syndrome cases associated to MSH2 characterization of a new deletion involving both EPCAM and the 5' part of MSH2. Cancer Prev Res 4: 1556-1562, 2011.

280. Edwards RA, Witherspoon M, Wang K, Afrasiabi K, Pham T, Birnbaumer L and Lipkin SM: Epigenetic repression of DNA mismatch repair by inflammation and hypoxia in inflammatory bowel disease-associated colorectal cancer. Cancer Res 69: 6423-6429, 2009

281. Winkler R, Witmer A and Heusermann H: Cancer and Crohn's disease. Z Gastroenterol 40: 569-576, 2002.

282. Nelson RL, Prasad ML and Abcarian H: Anal carcinoma presenting as a perirectal abscess or fistula. Arch Surg 120: 632-635, 1985

283. Yang BL, Shao WJ, Sun GD, Chen YQ and Huang JC: Perianal mucinous adenocarcinoma arising from chronic anorectal fistulae: a review from a single institution. Int J Colorectal Dis 24: 1001-1006, 2009.

284. Ribeiro ML, Priolli DG, Miranda DDC, Arçari DP, Pedrazzoli J Jr and Martinez CAR: Analysis of oxidative DNA damage in patients with colorectal cancer. Clin Colorectal Cancer 7: 267-272, 2008.

285. Eltzschig HK and Carmeliet P: Hypoxia and inflammation. N Eng J Med 364: 656-665, 2011.

286. Simiantonaki N, Taxeidis M, Jayasinghe C, Kurzik-Dumke U and Kirkpatrick CJ: Hypoxia-inducible factor 1 alpha expression increases during colorectal carcinogenesis and tumor progression. BMC Cancer 8: 320, 2008.

287. Slattery ML, Wolff RK, Herrick J, Caan BJ and Samowitz W: Tumor markers and rectal cancer: support for an inflammationrelated pathway. Int J Cancer 125: 1698-1704, 2009.

288. Alexeyev O, Bergh J, Marklund I, Thellenberg-Karlsson C, Wiklund F, Grönberg H, Bergh A and Elgh F: Association between the presence of bacterial 16S RNA in prostate specimens taken during transurethral and subsequent risk of prostate cancer. Cancer Causes Control 17: 1127-1133, 2006.

289. Cohen RJ, Shannon BA, McNeal JE, Shannon T and Garett KL: Propionibacterium acnes associated with inflammation in radical prostatectomy specimens: a possible link to cancer evolution? J Urol 173: 1969-1974, 2005.

290. Zhou Z, Hong L, Shen X, Rao X, Jin X, Lu G, Li L, Xiong E, Li W, Zhang J, Chen Z, Pan J and Song B: Detection of nanobacteria infection in type III prostatitis. Urology 71: 1091-1095, 2008. 
291. Shen X, Ming A, Li X, Zhou Z and Song B: Nanobacteria: a possible etiology for type III prostatitis. J Urol 164: 364-369, 2010.

292. Martel J and Young JD: Purported nanobacteria in human blood as calcium carbonate nanoparticles. Proc Natl Acad Sci USA 105: 5549-5554, 2008.

293. De Nunzio C, Kramer G, Marberger M, Montironi R, Nelson W, Schröder F, Sciarra A and Tubaro A: The controversial relationship between benign prostatic hyperplasia and prostate cancer: the role of inflammation. Eur Urol 60: 106-117, 2011.

294. Liu Y, Mo JQ, Hu Q, Boivin G, Levin L, Lu S, Yang D, Dong Z and $\mathrm{Lu} \mathrm{S}$ : Targeted overexpression of vav3 oncogene in prostatic epithelium induces nonbacterial prostatitis and prostate cancer. Cancer Res 68: 6396-6406, 2008

295. Liu Y, Wu X, Dong Z and Lu S: The molecular mechanism of Vav3 oncogene on upregulation of androgen receptor activity in prostate cancer cells. Int J Oncol 36: 623-633, 2010.

296. Dong Z, Liu Y, Lu S, Wang A, Lee K, Wang LH, Revelo M and Lu S: Vav3 oncogene is overexpressed and regulates cell growth and androgen receptor activity in human prostate cancer. Mol Endocrinol 20: 2315-2325, 2006.

297. Lee K, Liu Y, Mo JQ, Zhang J, Dong Z and Lu S: Vav3 oncogene activates estrogen receptor and its overexpression may be involved in human breast cancer. BMC Cancer 8: 158, 2008

298. Lyons LS and Burnstein KL: Vav3, a Rho GTPase guanine nucleotide exchange factor, increases during progression to androgen-independence in prostate cancer cells and potentiates androgen receptor transcriptional activity. Mol Endocrinol 20 1061-1072, 2006

299. Grant GE, Rubino S, Gravel S, Wang X, Patel P, Rokach J and Powell WS: Enhanced formation of 6-oxo-6,8,11,14-eicosatetraenoic acid by cancer cells in response to oxidative stress, docosahexaeonic acid and neutrophil-derived 5-hydroxyl-6,8.11,14eicosatetraenoic acid. Carcinogenesis 32: 822-828, 2011.

300. Karan D, Holzbeierlein J and Thrasher VB: Macrophage inhibitory cytokine-1: possible bridge molecule of inflammation and prostate cancer. Cancer Res 69: 2-5, 2009.

301. Kwon EM, Salinas CA, Kolb S, Fu R, Feng Z, Stanford JL and Ostrander EA: Genetic polymorphism in inflammation pathway genes and prostate cancer. Cancer Epidemiol Biomarkers Prev 20: 923-933, 2011.

302. Thomas LN, Morehouse TJ and Too CK: Testosterone and prolactin increase carboxypeptidase-D and nitric oxide levels to promote survival of prostate cancer cells. Prostate doi: 10.1002/ pros.21446, 2011

303. Baritaki S, Huerta-Yepez S, Sahakyan A, Karagiannides I, Bakirtzi K, Jazirehi A and Bonavida B: Mechanisms of nitric oxide-mediated inhibition of EMT in cancer: inhibition of the metastasis-inducer Snail and induction of the metastasis suppressor RKIP. Cell Cycle 9: 4931-4940, 2010.

304. Sharad S, Srivastava A, Ravulapali S, Parker P, Chen Y, Li H, Petrovics $G$ and Dobi A: Prostate cancer gene expression signature of patients with high body mass index. Prostate Cancer Prostatic Dis 14: 22-29, 2011.

305.Zhau HE, He H, Wang CY, Zayzafoon M, Morrissey C, Vessella RL, Marshall FF, Chung LW and Wang R: Human prostate cancer harbors the stem cell properties of bone marrow mesenchymal stem cells. Clin Cancer Res 17: 2159-2169, 2011.

306. Martin P, Liu YN, Pierce R, Abou-Kheir W, Casey O, Seng V, Camacho D, Simpson RM and Kelly K: Prostate epithelial Pten/ TP53 loss leads to transformation of multipotential progenitors and epithelial to mesenchymal transition. Am J Pathol 179: 422-435, 2011.

307. Mak P, Leav I, Pursell B, Bae D, Yang X, Tagllienti CA, Gouvin LM, Sharma VM and Mercurio AM: ERbeta impedes prostate cancer EMT by destabilizing HIF-1alpha and inhibiting VEGF-mediated snail nuclear localization: implications for Gleason grading. Cancer Cell 17: 319-332, 2010.

308. Armstrong J, Marengo MS, Oltean S, Kemeny G, Bitting RL, Turnbull JD, Harold CI, Marcom PK, George DJ and GarciaBlanco MA: Circulating tumor cells from patients with advanced prostate and breast cancer display both epithelia and mesenchymal markers. Mol Cancer Res 9: 997-1007, 2011a.

308. Baritaki S, Chapman A, Yeung K, Spandidos DA, Palladino M and Bonavida B: Inhibition of epithelial to mesenchymal transition in metastatic prostate cancer cells by the novel proteasome inhibitor, NPI-0052: pivotal roles of Snail repression and RKIP induction. Oncogene 28: 3573-3585, $2008 \mathrm{~b}$.
309. Peng X, Guo W, Liu T, Wang X, Tu X, Xiong D, Chen S, Lai Y, Du H, Chen G, Liu G, Tang Y, Huang S and Zou X: Identification of miRs-143 and -145 that is associated with bone metastasis of prostate cancer and involved in the regulation of EMT. PLoS One 6: e20341, 2011.

310. Kaddar T, Rouault JP, Chien WW, Chebel A, Gadoux M, Salles G, Ffrench M and Magaud JP: Two new miR-16 targets: caprin-1 and HMGA-1, proteins implicated in cell proliferation. Biol Cell 101: 511-524, 2009.

311. Wang L, Tang H, Thayanithy V, Subramanian S, Oberg AL, Cunningham JM, Cerhan JR, Steer CJ and Thibodeau SN: Gene networks and microRNAs implicated in aggressive prostate cancer. Cancer Res 69: 9490-9497, 2009.

312. Albadine R, Latour M, Oubaji A, Haffner M, Isaacs WB, Platz A, Meeker AK, Demarzo AM, Epstein JI and Netto GJ: TMPRSS2-ERG gene fusion status in minute (minimal) prostatic adenocarcinoma. Mol Pathol 22: 1415-1422, 2009.

313. Mani RS, Iyer MK, Cao Q, Brenner JC, Wang L, Ghosh A, Cao X, Lonigro RJ, Tomlins SA, Varambally S and Chinnaiyan AM: TMPRSS-ERG-mediated feed-forward regulation of wild-type ERG in human prostate cancers. Cancer Res 71: 5387-5392, 2011.

314. Helgeson BE, Tomlins SA, Shah N, Laxman B, Cao Q, Ptensner JR, Cao X, Singla N, Montie JE, Varabally S, Mehra R and Chinnaiyan AM: Characterization of TMPRSS2:ETV5 and SLC5A3:ETV5 gene fusions in prostate cancer. Cancer Res 68: 73-80,2008

315. Kämmerer U, Germeyer A, Stengel S, Kapp M and Denner J: Human endogenous retrovirus K (HERV-K) is expressed in villous and extravillous cytotrophoblast cells of the human placenta. J Reprod Immunol 91: 1-8, 2011.

316. Toubaji A, Albadine R, Meeker AK, Isaacs WB, Lotan T, Haffner MC, Chaux A, Epstein JI, Han M, Walsh PC, Partin AW, De Marzo AM, Platz EA and Netto GJ: Increased gene copy number of ERG on chromosome 21 but not TMPRSS2-ERG fusion predicts outcome in prostatic adenocarcinoma. Mol Pathol doi: 10.1038/molpathol.2011.111, 2011.

317. Foster CS, Dodson AR, Ambroisine L, Fisher G, Meller H, Clark H, Attars G, De Bono J, Scardino P, Reuter VE, Cooper CS, Berney DM and Cuzick J: Hsp-27 expression at diagnosis predicts poor clinical outcome in prostate cancer independent of ETS-gene rearrangement. Br J Cancer 101: 1137-1144, 2009.

318. Hollenhorst PC, Paul L, Ferris MW and Graves BJ: The ETS gene ETV4 is required for anchorage-independent growth and a cell proliferation gene expression program in $\mathrm{PC} 3$ prostate cells. Genes Cancer 1: 1044-1052, 2011.

319. Sakakibara S, Sakakibara K and Tosato G: NF-[kappa]B activation stimulates transcription and replication of retrovirus XMRV in human B-lineage and prostate carcinoma cells. J Virol 85: 3179-3186, 2011

320. Paprotka T, Delviks-Frankenberry KA, Cingöz O, Martinez A, Kung HJ, Tepper CG, Hu WS, Fivash MJ Jr, Coffin JM and Pathak VK: Recombinant origin of the retrovirus XMRV. Science 333: 97-101, 2011.

321. Sinkovics JG, Györkey F, Kusyk C and Siciliano MJ: Growth of human tumor cells in established cultures. Methods Cancer Res XIV: 243-323, 1978.

322. Knox K, Carrigan D, Simmons G, Teque F, Zhou Y, Hackett J Jr, Qiu X, Luk KC, Schochetman G, Knox A, Kogelnik AM and Levy JA: No evidence of murine-like gammaretroviruses in CFS patients previously identified as XMRV-infected. Science 333: 94-97, 2011

323. Lukacs RU, Memarzadeh S, Wu H and Witte ON: Bmi-1 is crucial regulator of prostate stem cell self-renewal and malignant transformation. Cell Stem Cell 7: 682-693, 2010.

324. Zhao J, Luo XD, Da CL and Xin Y: Clinicopathological significance of B-cell-specific Moloney murine leukemia virus insertion site 1 expression in gastric carcinoma and its precancerous lesion. World J Gastroenterol 15: 2145-2150, 2009.

325. Huang YW, Wang LS, Chang HL, Ye W, Shu S, Sugimoto Y and Lin YC: Effect of keratinocyte growth factor on cell viability in primary cultured human prostate cancer stromal cells. J Steroid Biochem Mol Biol 100: 24-33, 2006.

326. Mehta PB, Robson CN, Neal DE and Leung HY: Keratinocyte growth factor activates $\mathrm{p} 38$ MAPK to induce stress fibre formation in human prostate DU145 cells. Oncogene 20: 5359-5365, 2001a.

326. Mehta PB, Robson CN, Neal DE and Leung HY: Serum keratinocyte growth factor measurement in patients with prostate cancer. J Urol 164: 2151-2155, 2000 b.

327. Heer R, Collins AT, Robson CN, Shenton BK and Leung HY: KGF suppresses $\alpha 2 \beta 1$ integrin function and promotes differentiation. Cell Sci 119: 1416-1424, 2006. 
328. Planz B, Aretz HT, Wang Q, Tabatabaei S, Kirley SD, Lin CW and McDougal WS: Immunolocalization of the keratinocyte growth factor in benign and neoplastic human prostate and its relation to androgen receptor. Prostate 41: 233-242, 1999a.

328. Planz B, Oltean H, Deix T, Kirley SD, Wang QF, McDougal WS and Marberger M: Effect of keratinocyte growth factor and activin on cell growth in the human prostatic cancer cell line LNCaP. World J Urol 22: 140-144, 2004b.

329. Crescioli C, Maggi M, Luconi M, Vanneli GB, Salerno R, Sinisi AA, Bonaccorsi L, Ferruzzi P, Barni T, Forli G and Serio M: Vitamin D3 analogue inhibits keratinocyte growth factor signaling and induces apoptosis in human prostate cancer cells. Prostate 50: 15-26, 2002.

330. Marchiani S, Bonaccorsi L, Ferruzzi P, Crescioli C, Muratori M, Adorini L, Forti G, Maggi M and Baldi E: The vitamin D analogue BXL-628 inhibits growth factor-stimulated proliferation and invasion of DU145 prostate cancer cells. J Cancer Res Clin Oncol 131: 408-416, 2006.

331. Culig Z, Hobisch A, Cronauer MV, Radmayr C, Trapman J, Hittmair A, Bartsch G and Klocker H: Androgen receptor activation in prostatic tumor cell lines by insulin-like growth factor-I, keratinocyte growth factor and epidermal growth factor. Eur Urol 27 (Suppl 2): 45-47, 1995

332. Dahiya R, Lee C, Haughney PC, Chui R, Ho R and Deng G: Differential gene expression of transforming growth factor alpha and beta, epidermal growth factor, keratinocyte growth factor, and their receptors in fetal and adult human prostatic tissues and cancer cell lines. Urology 48: 963-970, 1995.

333. McGarvey TW and Stearns ME: Keratinocyte growth factor and receptor mRNAs expression in benign and malignant human prostate. Exp Med Pathol 63: 52-62, 1995

334. Leung HY, Mehta P, Gray LB, Collins AT, Robson CN and Neal DE: Keratinocyte growth factor expression in hormone insensitive prostate cancer. Oncogene 15: 1115-1120, 1997.

335. Vignale RA, Espasandin J, Paciel J and Scarrone V: Diagnostic value of keratosis palmaris as indicative sign of visceral cancer. Med Cutan Ibero Lat Am 11: 287-292, 1983

336. Fu J, Qu Z, Yan P, Ishikawa C, Ageilan RI, Rabson AB and Xiao G: The tumor suppressor gene WWOX links the canonical and noncanonical NFאB pathways in HTLV-I Tax-mediated tumorigenesis. Blood 117: 1652-1661, 2011.

337. Kurek K, Del Mare S, Salah Z, Abdeen S, Sadig H, Lee S, Gaudio E, Zanesi N, Jones KB, De Yong B, Amir G, Gebhardt M, Warman M, Stein GS, Stein JB and Aqeilan EI: Frequent attenuation of the WWOX tumor suppressor in osteosarcoma is associated with increased tumorigenicity and aberrant RUNX2 expression. Cancer Res 70: 5577-5586, 2010.

338. Mare SD, Kurek KC, Stein GS, Lian JB and Aqeilan RI: Role of the WWOX tumor suppressor gene in bone homeostasis and the pathogenesis of osteosarcoma. Am J Cancer Res 15: 585-594, 2011.

339. Iliopoulos D, Fabbri M, Druck T, Qin HR, Han SY and Huebner K: Inhibition of breast cancer cell growth in vitro and in vivo: effect of restoration of Wwox expression. Clin Cancer Res 13: 268-274, 2007.

340. Sudol M: Newcomers to the WW domain-mediated network of the hippo tumor suppressor pathway. Genes Cancer 1: 1115-1118, 2010.

341. Zelazowski MJ, Pluciennik E, Pasz-Walczak G, Potemski P, Kordek R and Bednarek AK: WWOX expression in colorectal cancer - a real-time quantitative RT-PCR study. Tumour Biol 32: $551-560,2011$

342. Kimura M, Takenobu H, Akita N, Nakazawa A, Ochial H, Shimozato O, Fujimura Y, Koseki H, Yoshiro I, Kimura H, Nakagawa A and Kamijo T: Bmil regulates cell fate via tumor suppressor WWOX repression in small-cell lung cancer cells. Cancer Sci 102: 983-990, 2011.

343. Qin HR, Iliopoulos D, Nakamura T, Costinean S, Volinia S, Druck T, Sun J, Okumura H and Huebner K: WWOX suppresses prostate cancer cell growth through modulation of Erb2-mediated androgen receptor signaling. Mol Cancer Res 5: 957-965, 2007.

344. Cai C, Chen S, Ng P, Bubley GJ, Nelson PS, Mostaghel EA, Marck B, Matsumoto AM, Simon N, Wang H, Chen S and Balk SP: Intratumoral de novo steroid synthesis activates androgen receptor in castration resistant prostate cancer and is upregulated by treatment with CYP17A1 inhibitors. Cancer Res 2011 [Epub ahead of print].

345. Lubik AA, Gunter JH, Hendy SC, Locke JA, Adomat HH, Thompson V, Herington A, Gleave ME, Pollak M and Nelson CC: Insulin increases de novo steroidogenesis in prostate cancer cells. Cancer Res 71: 5754-5764, 2011.
346. Darimipourain M, Wang S, Ittmann M and Kwabi-Addo B: Transcriptional and post-transcriptional regulation of Sproutyl, a receptor tyrosine kinase inhibitor in prostate cancer. Prostate Cancer Prostatic Dis doi: 10.1036/pcan.2011.33, 2011.

347. Kouprina N, Pavlicek A, Noskov VN, Solomon G, Otstot J, Isaacs W, Carpenter JD, Trent JM, Schleutker J, Barrett JC, Jurka $J$ and Larionov V: Dynamic studies of the SPANX gene cluster mapped to the prostate cancer susceptibility locus HPCX at Xq27. Genome Res 15: 1477-1486, 2005.

348. Salem M, Calogero AE, Zaccarello G, Castiglione R, Cosentino A, Campagna C, Vicari E and Rappazzo G: Expresssion of SPANX proteins in normal prostatic tissue and in prostate cancer. Eur J Histochem 54: e41, 2010.

349. Mattila H, Schindler M, Isotalo J, Ikonen T, Vihinen M, Oja H, Tammela TL, Wahlfors T and Schleutker J: NMD and microRNA expression profiling of the HPOX1 locus reveal MAGE1 as a candidate prostate cancer predisposition gene. BMC Cancer 11: 327, 2011.

350. Wilson EM: Androgen receptor molecular biology and potential targets in prostate cancer. Ther Adv Urol 2: 105-117, 2010a.

350. Vitichié G, Lena AM, Latina A, Formosa A, Gregersen LH, Lund AH, Bernardini S, Mauriello A, Miano R, Spagnoli LG, Knight RA, Candi E and Melino G: MiR-203 controls proliferation, migration and invasive potential of prostate cancer cell lines. Cell Cycle 10: 1121-1131, $2011 \mathrm{~b}$.

351. Szczyrba J, Nolte E, Wach S, Kremmer E, Stöhr R, Hartmann A, Wieland W, Wullich B and Grässer FA: Downregulation of Sec23A protein by miRNA-375 in prostate carcinoma. Mol Cancer Res 9: 791-800, 2011

352. Lin DW, Fitzgerald LM, Fu R, Kwon EM, Zheng SL, Kob S, Wiklund F, Stattin P, Isaacs WB, Xu J, Ostrander EA, Feno Z, Grönberg H and Stanford JL: Genetic variants in the LEPR, CRY1, RNASEL, IL-4, and ARVCF genes are prognostic markers of prostate cancer-specific mortality. Cancer Epidemiol Biomarkers Prev 20: 1928-1936, 2011.

353. Zhao ZM, Reynolds AB and Gaucher EA: The evolutionary history of the catenin gene family during metazoan evolution. BMC Evol Biol 11: 198, 2011.

354. Perry AS, Watson WG, Lawler M and Hollywood D: The epigenome a therapeutic target in prostate cancer. Nat Rev Urol 7: $668-680,2010$

355. Gonzales JC, Fink LM, Goodman OB Jr, Symanowski JT, Vogelzang NJ and Ward DC: Comparison of circulating microRNA 141 to circulating tumor cells, lactic dehydrogenase, and prostatespecific antigen for determining treatment response in patients with metastatic prostate cancer. Clin Genitourin Cancer 9: 39-45, 2011.

356. Magenta A, Cencioni C, Fasanaro P, Zaccagnini G, Greco S, Sarra-Ferraris G, Antonini A, Martelli F and Capogrossi MC miR-200c is upregulated by oxidative stress and induces endothelial cell apoptosis and senescence via ZEB1 inhibition. Cell Death Differ 18: 1628-1639, 2011.

357. Smith CM, Watson DI, Leong MP, Mayne GC, Michaelk MZ, Winhoven BP and Hussey DJ: miR-200 family expression is downregulated upon neoplastic progression of Barrett's esophagus. World J Gastroenterol 17: 1036-1044, 2011.

358. Wang H, Leav I, Ibaragi S, Wegner M, Hu GF, Lu ML, Balk SP and Yuan X: SOX9 is expressed in human fetal prostate epithelium and enhances prostate cancer invasion. Cancer Res 68: 1625-1630, 2008.

359. Amaral PP, Neyt C, Wilkins SJ, Askarian-Amiri ME, Sunkin SM, Perkins AC and Mattick JS: Complex architecture and regulated expression of the Sox 2 ot locus during vertebrate development. RNA 15: 2013-2027, 2009.

360. Lengerke C, Fehm T, Kurth R, Neubauer H, Scheble V, Müller F, Schneider F, Petersen K, Wallwiener D, Kanz L, Fend F, Perner S, Bareiss PM and Staebler A: Expression of the embryonic stem cell marker SOX2 in early-stage breast carcinoma. BMC Cancer 11: 42, 2011

361. Hubbard SA, Friel AM, Kumar B, Zhang L, Rueda BR and Gargett CE: Evidence for cancer stem cells in human endometrial carcinoma. Cancer Res 69: 8241-8248, 2009.

362. Huang YW, Liu JC, Deatherage DE, Luo J, Mutch DG, Goodfellow PJ, Miller DS and Huang TH: Epigenetic repression of microRNA-129-2 leads to overexpression of SOX4 oncogene in endometrial cancer. Cancer Res 69: 9038-9046, 2009.

363. Jia X, Li X, Xu Y, Zhang S, Mou W, Liu Y, Liu Y, Lv D, Liu CH, Tan X, Xiang R and Li N: SOX2 promotes tumorigenesis and increases the anti-apoptotic property of human prostate cancer cell. J Mol Cell Biol 3: 230-238, 2011. 
364. Baniwal SK, Khalid O, Gabet Y, Shah RR, Purcell DJ, Mav D, Kohn-Gabet AE, Shi Y, Coetze GA and Frenkel B: Runx2 transcriptome of prostate cancer cells: insights into invasiveness and bone metastasis. Mol Cancer 9: 258, 2010.

365. Pratap J, Lian JB and Stein GS: Metastatic bone disease: role of transcription factors and future targets. Bone 48: 30-36, 2011.

366. Van der Horst G, van den Hoogen C, Buijs JT, Cheung H, Bloys H, Pelger RCM, Lofrenzon G, Heckmann B, Feyen J, Pujuguet O, Blanque R, Clément-Lacroix P and van der Pluij G: Targeting of $\alpha \mathrm{v}$-integrins in stem/progenitor cells and supportive microenvironment impairs bone metastasis in human prostate cancer. Neoplasia 13: 516-525, 2011

367. Blyth K, Cameron ER and Nail JC: The RUNX genes: gain or loss of function in cancer. Nat Rev Cancer 5: 376-387, 2005.

368. Rudra D, Egawa T, Chong MMW, Treutig P, Littman DR and Rudensky AY: RUNX-CBF $\beta$ complexed control Foxp3 expression in regulatory T cells. Nat Immunol 120: 1170-1177, 2009.

369. Naoe Y, Setoguchi R, Akiyama K, Muroi S, Kuroda M, Hatam F, Littman DR and Taniuchi I: Repression of interleukin-4 in $\mathrm{T}$ helper type 1 cells by Runx/Cbf $\beta$ binding to the IL- 4 silencer. J Exp Med 204: 1749-1755, 2007.

370. Tsourdi E, Rachner TD, Rauner M, Hamann C and Hofbauer L: Denosumab for bone diseases - translating bone biology into targeted therapy. Eur J Endocrinol 2011 [Epub ahead of print].

371. May KF Jr, Gulley JL, Drake CG, Dranoff G and Kantoff PW: Prostate cancer immunotherapy. Clin Cancer Res 17: 5233-5238, 2011.

372. Stein WD, Gulley JL, Schlom J, Madan RA, Dahut W, Figg WD, Ning YM, Arien PM, Price D, Bates SE and Fojo T: Tumor regression and growth rates determined in five intramural NCI prostate cancer trials: the growth rate constant as an indicator of therapeutic efficacy. Clin Cancer Res 17: 907-917, 2011.

373. Satoh M, Wang H, Ishidoya S, Abe H, Mofriya T, Hamada $H$ and Arai Y: Oncolytic virotherapy for prostate cancer by E1A.E1B mutant adenovirus. Urology 70: 1243-1248, 2007.

374. Sandberg L, Papareddy P, Silver J, Bergh A and Mei YF: Replication-competent Ad11p vector (RCAd11p) efficiently transduces and replicates in hormone-refractory metastatic prostate cancer cells. Hum Gene Ther 20: 361-373, 2009.

375. Msaouel P, Iankov ID, Allen C, Morris JC, von Messling V, Cattaneo R, Koutsilieris M, Russell SJ and Galanis E: Engineered measles virus as a novel oncolytic therapy against prostate cancer. Prostate 69: 82-91, 2009.

376. Lee CY, Rennie PS and Jia WW: MicroRNA regulation of oncolytic herpes simplex virus 1 for selective killing of prostate cancer cells. Clin Cancer Res 15: 5126-5136, 2009.

377. Passer BJ, Wu CL, Wu S, Rabkin SD and Martuza RL: Analysis of genetically engineered oncolytic herpes simplex viruses in human prostate cancer organotypic cultures. Gene Ther 16: 1477-1482, 2009.

378. Fukuhara H, Homma Y and Todo T: Oncolytic virus therapy for prostate cancer. Int J Urol 17: 20-30, 2010.

379. Oberg D, Yanover E, Adam V, Sweeney K, Costas C, Lemoine NR and Halldén G: Improved potency and selectivity of an oncolytic E1ACR2 and E1B19K deleted adenoviral mutant in prostate and pancreatic cancers. Clin Cancer Res 16: 541-553, 2010.

380. Moussavi M, Fazli L, Tearle H, Guo Y, Cox M, Bell J, Ong C, Jia W and Rennie PS: Oncolysis of prostate cancers induced by vesicular stomatitis virus in PTEN knockout mice. Cancer Res 70: 1367-1376, 2010

381. Lee CY, Bu LX, De Benedetti A, Williams BJ, Rennie PS and Jia WW: Transcriptional and translational dual-regulated oncolytic herpes simplex virus type 1 for targeting prostate tumors. Mol Ther 18: 929-935, 2010

382. Castelo-Branco P, Passer BJ, Buhrman JS, Antszczyk S, Marinelli M, Xaupa C, Rabkin SD and Martuza RL: Oncolytic herpes simplex virus armed with xenogeneic homologue of prostate acid phosphatase enhances antitumor efficacy in prostate cancer. Gene Ther 17: 805-810, 2010.

383. Gentscev I, Donat U, Hofmann E, Weibel S, Adelfinger M, Raab V, Heisig M, Chen N, Yu YA, Stritzker J and Szalay AA: Regression of human prostate tumors and metastases in nude mice following treatment with the recombinant oncolytic vaccinia virus GLV-1h68. J Biomed Biotechnol 2010: 489759, 2010.

384. Radhakrishnan S, Miranda E, Ekblad M, Holford A, Pizarro MT, Lemoine NR and Halldén G: Efficacy of oncolytic mutants targeting $\mathrm{pRb}$ and $\mathrm{p} 53$ pathways is synergistically enhanced when combined with cytotoxic drugs in prostate cancer cells and tumor xenografts. Hum Gene Ther 21: 1311-1325, 2010.

385. Ekblad M and Halldén G: Adenovirus-based therapy for prostate cancer. Curr Opin Mol Ther 12: 421-431, 2010.
386. Muthana M, Giannoudis A, Scott SD, Fang HY, Offelt SB Morrow FJ, Murdoch G, Burton J, Cross N, Burke B, Mistry R, Hamdy F, Brown NJ, Gregopoulos L, Hoskin P, Essand M, Lewis CE and Maitland NJ: Use of macrophages to target therapeutic adenovirus to human prostate tumors. Cancer Res 71: $1805-1815,2011$

387. Gujar SA, Pan DA, Marcato OP, Garant KA and Lee PW: Oncolytic virus-initiated protective immunity against prostate cancer. Mol Ther 19: 797-804, 2011.

388. Echchgadda I, Chang TH, Sabbah A, Bakri I, Ikeno Y, Hubbard GB, Chatterjee B and Bose S: Oncolytic targeting of androgen-sensitive prostate tumor by the respiratory syncytial virus (RSV): consequences of deficient interferon-dependent antiviral defense. BMC Cancer 11: 43, 2011.

389. Lavaille S, Samuel S, Goulet ML and Hiscott J: Enhancing VSV oncolytic activity with an improved cytosine deaminase suicide gene strategy. Cancer Gene Ther 128: 435-443, 2011.

390. Savarese F, Dávila A, Nechanitzky R, De La Rosa-Velazquez I, Pereira CF, Engelke R, Takahashi K, Jenuwein T, KohwiShigematsu T, Fisher AG and Grosschedl R: Satb1 and Satb2 regulate embryonic stem cell differentiation and Nanog expression. Genes Dev 23: 2625-2638, 2009.

391. McInnes N, Sadlon TJ, Brown CY, Pederson S, Beyer M, Schulze JL, McColl S, Goodall GJ and Barry SC: FOXP3 and FOXP3-regulated microRNAs suppress STATB1 in breast cancer cells. Oncogene doi: 10.1038/onc.2011.293, 2011.

392. Schalken JA, Hessels D and Verhaegh G: New targets for therapy in prostate cancer: differential display code 3 (DD3(PCA3)), a highly prostate cancer-specific gene. Urology 62 (Suppl): 34-43, 2003.

393. Klatte T, Waldert M, De Martino M, Schatzl G, Mannhalter C and Remzi M: Age-specific PCA3 score reference values for diagnosis of prostate cancer. World J Urol 2011 [Epub ahead of print].

394. Mao LJ, Zheng JN, Li W, Wang JQ, Chen JC and Sun XQ: Construction of an oncolytic adenovirus expressing small hairpin RNA and targeting the SATB1 gene. Zhonghua Nan Ke Xue 16: 679-683, 2010.

395. Berger MF, Lawrence MS, Demichelis F, Drier Y, Cibulskis K, Sivachenko AY, Sboner A, Esgueva R, Pflueger D, Sougnez C, Onofrio R, Carter SL, Park K, Habegger L, Ambrogio L, Fennell T, Parkin M, Saksena G, Voet D, Ramos AH, Pugh TJ, Wilkinson J, Fisher S, Winckler W, Mahan S, Ardlie K, Baldwin J, Simons JW, Kitabayashi N, MacDonald TY, Kantoff PW, Chin L, Gabriel SB, Gerstein MB, Golub TR, Meyerson M, Tewari A, Lander ES, Getz G, Rubin MA and Garraway LA: The genomic complexity of primary human prostate cancer. Nature 470: 214-220, 2011

396. Peraldo-Neia C, Migliardi G, Mello-Grand M, Montemurro F, Segir R, Pignochino Y, Cavalloni G, Torchio B, Mosso L, Chiorino $\mathrm{G}$ and Aglietta $\mathrm{M}$ : Epidermal growth factor receptor (EGFR) mutation analysis, gene expression profiling and EGFR protein expression in primary prostate cancer. BMC Cancer 11: 31, 2011

397. Isebaert SF, Swinnen JV, McBride WH and Haustermans RML: Insulin-like growth factor type 1 receptor inhibitor BNVPAEW541 enhances radiosensitivity of PTEN wild-type but not PTEN-deficient human prostate cancer cells. Int J Radiat Oncol Biol Phys 81: 239-247, 2011

398. Himpe E, Potikanond S, Verdood P and Kooijman R: Attenuation of IGF-1 receptor signaling inhibits serum-induced proliferation of prostate cancer cells. Growth Horm IGF Res 21: 252-259, 2011

399. Tam NN, Szeto CY, Freundenberg JM, Fullenkamp AN, Medvedovic M and Ho SM: Research resource: estrogen-driven prolactin-mediated gene-expression networks in hormoneinduced prostatic intraepithelial neoplasia. Mol Endocrinol 24: 2207-2217, 2010.

400. Planche A, Bacac M, Provero P, Fusco C, Delorenzi M, Stehle JC and Stamenkovic I: Identification of prognostic molecular feature in the reactive stroma of human breast and prostate cancer. PLoS One 6: e18640, 2011.

401. Martinez AA, Gonzalez J, Hong Y, Ghilezan M, Shetty S, Kernen K, Gustafson G, Krauss D, Vicini F and Kestin L: Dose escalation improves cancer-related events at 10 years for intermediate- and high-risk prostate cancer patients treated with hypofractionated high dose boost and external beam radiotherapy. Int J Radiat Oncol Biol Phys 79: 363-370, 2011.

402. Ghilezan M, Galalae R, Demanes J, Schour L, Gustafson G, Nuernberg N, Wallace $M$ and Martinez A: 10 year results in 1577 intermediate/high risk prostate cancer patients treated with external beam RT (EBRT) and hypofractionated high dose rate (HDR) brachytherapy boost. Proc 49th ASTRO Meeting. Int J Radiat Oncol Biol Phys 69: S83-S84, abs. 147, 2007. 
403. Biagioli MC and Hoffe SE: Emerging technologies in prostate cancer radiation therapy: improving the therapeutic window. Cancer Control 17: 223-232, 2010.

404. Sountoulides P, Koletsas N, Kikidakis D and Paschalidis K: Secondary malignancies following radiotherapy for prostate cancer. Ther Adv Urol 2: 119-125, 2010.

405. Kaufman DS, Zietman AL, Dahl DM, Harisinghani MG and Wu CL: Case records of the Massachusetts General Hospital. Case 5-2011. A 65-year-old man with hematuria after treatment for prostate cancer. N Engl J Med 364: 667-675, 2011.

406.Fortson WS, Kayarthodi S, Fujimura Y, Xu H, Matthews R, Grizzle WE, Rao VN, Bhat GK and Reddy ES: Histone deacetylase inhibitors, valproic acid and trichostatin-A induce apoptosis and affect acetylation status of p53 in ERG-positive prostate cancer cells. Int J Oncol 39: 111-119, 2011.

407. Abdulla A and Kapoor A: Emerging novel therapies in the treatment of castrate-resistant prostate cancer. Can Urol Assoc J 5: 120-133, 2011.

408. Araujo J and Logothetis C: Dasatinib: a potent SRC inhibitor in clinical development for the treatment of solid tumors. Cancer Treat Rev 36: 492-500, 2010.

409. George D and Moul JW: Emerging treatment options for patients with castration-resistant prostate cancer. Prostate doi: 10.1002/ pros.21435, 2011.

410. Inoda S, Hirohashi Y, Torigue T, Morita R, Takahashi A, Asanuma H, Nakatsugawa M, Nishizawa S, Tamura Y, Tsuruma T, Terui T, Kondo T, Ishitani K, Hasegawa T, Hirata K and Sato N: Cytotoxic $\mathrm{T}$ lymphocytes efficiently recognize human colon cancer stem-like cells. Am J Pathol 178: 1805-1813, 2011.

411. Feldmann A, Stamova S, Bippes CC, Bartsch H, Wehner R, Schmitz M, Temme A, Cartellieri M and Bachmann M: Retargeting of $\mathrm{T}$ cells to prostate stem cell antigen expressing tumor cells: comparison of different antibody formats. Prostate 71 : 998-1011, 2011.

412. Abedinpour P, Barori VT, Welsh J and Borgström P: Regression of prostate tumors upon combination of hormone ablation therapy and celecoxib in vivo. Prostate 71: 813-823, 2011.

413. Modugno F, Ness RB, Chen C and Weiss NS: Inflammation and endometrial cancer: a hypothesis. Cancer Epidemiol Biomarkers Prev 14: 2840-2847, 2005.

414. Wallace AE, Gibson DA, Saunders PT and Jabbour HN: Inflammatory events in endometrial adenocarcinoma. J Endocrinol 206: 141-157, 2010.

415. Cui Y, Borysova MK, Johnsom JO and Guadagno TM: Oncogenic $\mathrm{B}-\mathrm{Raf}^{\mathrm{v} 600 \mathrm{E}}$ induces spindle abnormalities, supernumerary centrosomes, and aneuploidy in human melanocytic cells. Cancer Res 70: 675-684, 2010.

416. Luria LE, Proper S and Sinkovics JG: Malignant melanoma: an approach to diagnosis and treatment. In: Advances in Plastic and Reconstructive Surgery. Woods JE (ed). Vol 124, pp63-110, 1997.

417. Sinkovics JG, Plager C, McMurtrey M, Papadopoulos NE, Waldinger R, Combs S, Romero J and Romsdahl MM: Adjuvant chemoimmunotherapy for malignant melanoma. In: Neoplasm Immunity: Experimental and Clinical. Crispen RG (ed). Elsevier North Holland, pp481-519, 1980.

418. Krone B, Kölmel KF, Henz BM and Grange JM: Protection against melanoma by vaccination with Bacille Calmette-Guerin (BCG) and/or vaccinia: an epidemiology-based hypothesis on the nature of a melanoma risk factor and its immunological control. Eur J Cancer 41: 104-117, 2005.

419. Muster T, Waltenberger A, Grassauer A, Hirschl S, Caucig P, Romirer I, Födinger D, Seppele H, SchanabO, Magin-Lachmann C, Löwer R, Jansen B, Pehamberger H and Wolff K: An endogenous retrovirus derived from human melanoma cells. Cancer Res 63: 8735-8741, 2003.

420. Schiavetti F, Thonnard J, Colau D, Boon T and Coulte PG: A human endogenous retroviral sequence encoding an antigen recognized on melanoma by cytolytic lymphocytes. Cancer Res 62: 5510-5516, 2002.

421. Schanab O, Humer J, Gleiss A, Mikula M, Sturlan S, Okamoto I, Muster T, Pehamberger $\mathrm{H}$ and Waltenberger A: Expression of human endogenous retrovirus $\mathrm{K}$ is stimulated by ultraviolet radiation in melanoma. Pigment Cell Melanoma Res 24: 656-665, 2011.

422. Sinkovics JG and Horvath JC: Vaccinations against human cancers. Int J Oncol 16: 81-96, 2000.

423. Cassel WA, Murray DR and Olkowski ZL: Newcastle virus oncolysate in the management of stage III malignant melanoma. In: Viral Therapy of Human Cancers. Sinkovics JG and Horvath JC (eds). Marcel Dekker, New York, pp667-689, 2005.
424. Eggermont AM: Immunostimulation versus immunosuppression after multiple vaccinations: the woes of therapeutic vaccine development. Clin Cancer Res 15: 6745-6747, 2009.

425. Nevala WK, Vachon CM,Leontovich AA, Scott CG, Thompson MA and Markovic SN: Evidence of systemic Th2-driven chronic inflammation in patients with metastatic melanoma. Clin Cancer Res 15: 1931-1939, 2009.

426. Martin-Orozco N, Li Y, Wang Y, Liu S, Hwu P, Liu Y-S, Dong C and Radvanyi L: Melanoma cells express ICOS ligand to promote the activation and expansion of T-regulatory cells. Cancer Res 70: 9581-9590, 2010.

427. Sinkovics JG and Horvath JC: Virological and immunological connotations of apoptotic and anti-apoptotic forces in neoplasia. Int J Oncol 19: 473-488, 2001. Erratum appeared in: Int J Oncol 27: 33,2005

428. Gaziel-Sovran A, Segura MF, Di Micco R, Collins MK, Hanniford D, Vega-Saenz de Miera E, Rakus JF, Dankert JF, Shang S, Kerbel RS, Bhardwaj N, Shao Y, Darvishian F, Zavadil J, Erlebacher A, Mahal LK, Osman I and Hernando E: miR-30b/30d regulation of GalNAc transferases enhances invasion and immunosuppression during metastases. Cancer Cell 20: 104-118, 2011

429. Sinkovics JG: Adult human sarcomas. I. Basic science. Expert Rev Anticancer Ther 7: 31-56, 2007.

430. Sinkovics JG: Adult human sarcomas. II. Medical oncology. Expert Rev Anticancer Ther 7: 183-210, 2007.

431. Sinkovics JG: Human sarcomas illustrated. In: Cytolytic Immune Lymphocytes. Schenk Buchverlag, Budapest, Passau, pp242-254, 2008.

432. Hoki Y, Murata M, Hiraku Y, Ma N, Matsumine A, Uchida A and Kawanishi S: 8-nitroguanine as a potential biomarker for progression of malignant fibrous histiocytoma, a model of inflammation-related cancer. Oncol Rep 18: 1165-1169, 2007.

433. Sinkovics JG: Cytotoxic lymphocytes against cellular and viral infectious agents. In: Cytolytic Immune Lymphocytes. Schenk Buchverlag, Budapest, Passau, pp149-214, 2008.

434. Sinkovics JG: Chondrosarcoma cell differentiation. Pathol Oncol Res 10: 174-187, 2004.

435. Nishida K, Kunisada T, Shen ZN, Kadota Y, Hashizume K and Ozaki T: Chondrosarcoma and peroxisome proliferator-activated receptor. PPAR Res 2008: 250568, 2008.

436. Dailey L, Laplantine E, Priore R and Basilico C: A network of transcriptional and signaling events is activated by FGF to induce chondrocyte growth arrest and differentiation. J Cell Biol 161: 1053-1068, 2003.

437. Khare S, Luc N, Dorfleutner A and Stehlik C: Inflammasomes and their activation. Crit Rev Immunol 30: 463-487, 2010.

438. Ruckdeschel JC, Codish SD, Stranahan A and McKneally MF: Postoperative empyema improves survival in lung cancer. Documentation and analysis of a natural experiment. $\mathrm{N}$ Engl $\mathrm{J}$ Med 287: 1013-1017, 1972a.

438. Bakker W, Nijhuis-Hedes JM, Wever AM, Brutel de la Riviere A, van der Velde EA and Dijkman JH: Postoperative intrapleural BCG in lung cancer: lack of efficacy and possible enhancement of tumor growth. Thorax 36: 870-874, 1981b.

439. Cho WC, Kwan CK, Yau S, So PP, Poon PC and Au JS: The role of inflammation in the pathogenesis of lung cancer. Expert Opin Ther Targets 15: 1127-1137, 2011.

440. Blanco D, Vicent S, Fraga MF, Fernandez-Garcia I, Freire J, Lulambio A, Esteller M, Ortiz-de-Solorzano C, Pio R, Lecanda E and Montuenga LM: Molecular analysis of a multistep lung cancer model induced chronic inflammation reveals epigenetic regulation of p16 and activation of the DNA damage response pathway. Neoplasia 9: 840-852, 2007.

441. Van den Heuvel MM, Burgers SA and van Zandwijk N: Immunotherapy in non-small-cell lung carcinoma: from inflammation to vaccination. Clin Lung Cancer 10: 99-105, 2009.

442. Sinkovics JG and Horvah JC: Evidence accumulating in support of cancer vaccines combined with chemotherapy: a pragmatic review of past and present efforts. Int J Oncol 29: 765-777, 2006.

443. Yoo J, Kang J, Lee HN, Aguilar B, Kafka D, Lee S, Choi I, Lee J, Ramu S, Haas J, Koh CJ and Hong YK: Kaposin-B enhances the PROX1 mRNA stability during lymphatic reprogramming of vascular endothelial cells by Kaposi's sarcoma herpes virus. PLoS Pathog 6: e10011046, 2010.

444. Sinkovics JG: DNA viruses. In: Cytolytic Immune Lymphocytes. Schenk Buchverlag, Budapest, Passau, pp187-214, 254-256, 2005 . 
445. Karki R, Lang SM and Means RE: The MARCH family E3 ubiquitin ligase K5 alters monocyte metabolism and proliferation through receptor tyrosine kinase modulation. PLoS Pathog 7: e1001331, 2011a.

445. Kang JG, Pripuzova N, Majerciak V, Kruhlak M, Le SY and Zheng ZM: Kaposi's sarcoma-associated herpesvirus ORF57 promotes escape of viral and human interleukin-6 from micro RNA-mediated suppression. J Virol 85: 2620-2630, 2011b.

446. Sirianni MC, Vincenzi L, Fiorelli V, Topino S, Scala E, Uccini S Angeloni A, Faggioni A, Cerimele D, Cottoni F, Aiuti F and Ensoli B: gamma-interferon production in peripheral blood mononuclear cells and tumor infiltrating lymphocytes from Kaposi's sarcoma patients: correlation with the presence of human herpesvirus- 8 in peripheral blood mononuclear cells and lesional macrophages. Blood 91: 968-976, 1998.

447. Schmidt K, Wies E and Neipel F: Kaposi's sarcoma-associated herpesvirus viral interferon regulatory factor 3 inhibits gamma interferon and major histocompatibility complex class II expression. J Virol 85: 4530-4537, 2011.

448. Lin X, Liang D, He Z, Deng Q, Robertsobn ES and Lan K: miRK12-7-5p encoded by Kaposi's sarcoma-associated herpesvirus stabilizes the latent state by targeting viral ORF50/RTA. PloS One 6: e16224, 2011.

449. Samols MA, Skalsky RL, Maldonado AM, Riva A, Lopez MC, Baker HV and Renne R: Identification of cellular genes targeted by KSHV-encoded microRNAs. PLoS Pathog 3: e65, 2007.

450. Skalsky RL, Samokla MA, Plaisance KB, Boss IW, Riva A, Lopez MC, Baker HV and Renne R: Kaposi's sarcoma-associated herpesvirus encodes an ortholog of miR-155. J Virol 81: 12836-12845, 2007

451. Ohira M, Seki N, Nadade T, Ishikawa K, Nomura N and Ohara O: Characterization of a human homolog $(\mathrm{BACH})$ of the mouse Bach1 gene encoding a BTB-basic leucine zipper transcription factor and its mapping to chromosome 21q22.1. Genomics 47 300-306, 1998.

452. Torres A, Torres K, Paszkowski T, Jodlowska-Jedrych B, Radomanski T, Ksiazek A and Maciejewski R: Major regulators of microRNAs biogenesis Dicer and Drosha are down-regulated in endometrial cancer. Tumour Biol 32: 769-776, 2011.

453. Warnatz HJ, Schmidt D, Manke T, Oiccini I, Sultan M,Borodina T, Baizerit D, Wruck W, Soldatov A, Vingron M, Lehrach H and Yaspo ML: The BTB and CNC homology 1 (BACH1) target genes are involved in the oxidative stress response and in control of the cell cycle. J Biol Chem 286: 23521-23532, 2011

454. Qin Z, Freitas E, Sullivan R, Mohan S, Baceieri R, Branch D, Romano M, Kearney P, Oates J, Plaisance K, Renne R, Kaleeba J and Parsons C: Upregulation of $\mathrm{xCT}$ by KSHV-encoded micro RNAs facilitates KSHV dissemination and persistence in an environment of oxidative stress. PLoS Pathog 6: e1000742, 2010.

455. Gregory SM, Davis BK, West JA, Taxman DJ, Matsuzawa S, Reed JC, Ting JP and Damania B: Discovery of a viral NLR homolog that inhibits the inflammasome. Science 331: 330-332, 2011.

456. Mesri EA: Inflammatory reactivation and angiogenicity of Kaposi's sarcoma-associated herpesvirus/HHV8: a missing link in the pathogenesis of acquired immunodeficiency syndromeassociated Kaposi's sarcoma. Blood 93: 4031-4033, 1999.

457. Goodier MR, Mela CM, Steel A, Gazzard B, Bower M and Gotch F: NKG2C ${ }^{+} \mathrm{NK}$ cells are enriched in AIDS patients with advanced-stage Kaposi's sarcoma. J Virol 81: 430-433, 2007.

458. Lambert M, Gannagé M, Karras A, Abel M, Legendre C, Kerob D, Agbalika F, Girard PM, Lebbe C and Caillat-Zucman S: Differences in the frequency and function of HHV8-specific CD8 T cells between asymptomatic HHV8 infection and Kaposi's sarcoma. Blood 106: 3871-3880, 2006.

459. Liang C, Lee JS and Jung JU: Immune evasion in Kaposi's sarcoma-associated herpes virus associated oncogenesis. Semin Cancer Biol 18: 423-436, 2008.

460. Sinkovics JG: Contradictory concepts in the etiology and regression of Kaposi's sarcoma. The Ferenc Györkey memorial lecture. Pathol Oncol Res 2: 249-267, 1996.

461. Tessari G, Naldi L, Boschiero L, Cordiano C, Piaserico S, Fortina AB, Cerimele D, La Oarola IL, Capuano M, Gotti F, Ruggenenti P, Sassi F, Remuzzi G and Girolomoni G: Incidence and clinical predictors of Kaposi's sarcoma among 1721 Italian solid organ transplant recipients: a multicenter study. Eur J Dermatol 16: 563-567, 2006.
462. Rodriguez-Peláez M, Fernández-Garcia MS, Gutiérrez-Corral N, De Francisco R, Riestra S, Garcia-Pravia C, Roriguez JI and Rodrigo L: Kaposi's sarcoma: an opportunistic infection by human herpesvirus- 8 in ulcerative colitis. J Crohns Colitis 4: 586-590, 2010.

463. Nichols LA, Adang LA and Kedes DH: Rapamycin blocks production of KSH/HHV8: insights into the anti-tumor activity of an immunosuppressant drug. PLoS One 6: e14535, 2011.

464. Niller HH, Wolff H, Ay E and Minarovits J: Epigenetic dysregulation of Epstein-Barr virus latency and development of autoimmune disease. Adv Exp Med Biol 711: 82-102, 2011.

465. Minarovits J, Hu LF, Imai S, Harabuchi Y, Kataura A, MinarovitsKormuta S, Osato T and Klein G: Clonality, expression and methylation patterns of the Epstein-Barr virus genomes in lethal midline granulomas classified as peripheral angiocentric $\mathrm{T}$ cell lymphomas. J Gen Virol 75: 77-84, 1994.

466. Mehta V, Balachandran C, Bhat S, Geretha V and Fernandes D: Nasal NKT cell lymphoma presenting as a lethal midline granuloma. Indian J Dermatol Venereol Leprol 74: 145-147, 2008.

467. Aozasa K and Zaki MA: Epiemiology and pathogenesis of nasal NK/T-cell lymphoma: a mini-review. Sci World J 11: 422-428, 2011.

468. Sinkovics JG and Horvath JC: Human natural killer cells: a comprehensive review. Int J Oncol 27: 5-47, 2005.

469. Nagy N and Klein E: Deficiency of the proaptotic SAP function in X-linked lymphoproliferative disease aggravates Epstein-Barr virus (EBV) induced mononucleosis and promotes lymphoma development. Immunol Lett 130: 13-18, 2010.

470. Kis LL, Takahara M, Nagy N, Klein G and Klein E: IL-10 can induce the expression of EBV-encoded latent membrane protein-1 (LMP-1) in the absence of EBNA-2 in B lymphocytes. Blood 107: 2928-2935, 2005.

471. Kis LL, Salamon D, Persson EK, Nagy N, Scheeren FA, Spits H, Klein G and Klein E: IL-21 imposes a type II EBV gene expression on type III and type I B cells by the repression of C- and activation of LMP-1 promoter. Proc Natl Acad Sci USA 107: 872-877, 2010.

472. Kis LL, Gerasimcik N, Salamon D, Persson EK, Nagy N, Klein G, Severinson E and Klein E: STAT signaling pathway activated by the cytokines IL-4 and IL-13 induces expression of the Epstein-Barr virus-encoded protein LMP-1 in absence of EBNA-2: implications for the type II EBV latent gene expression in Hodgkin lymphoma. Blood 117: 165-174, 2011.

473. Klein E, Kis LL and Takahara M: Pathogenesis of Epstein-Barr virus (EBV)-carrying lymphomas. Acta Microbiol Immunol Hung 53: 441-457, 2006.

474. Minárovits J: Microbe-induced epigenetic alterations in host cells: the coming era of patho-epigenetics of microbial infections: a review. Acta Microbiol Immunol Hung 56: 1-19, 2009.

475. Takacs M, Banati F, Koroknai A, Segesdi J, Salamon D, Wolf H, Niller HH and Minarovits J: Epigenetic regulation of latent Epstein-Barr virus promoters. Biochim Biophys Acta 1799: 228-235, 2010.

476. Zheng ZM: Viral oncogenes, noncoding RNAs, and RNAs splicing in human tumor viruses. Int J Biol Sci 6: 730-755, 2010.

477. Morgan RW and Burnside J: Roles of avian herpesvirus micro RNAs in infection, latency and oncogenesis. Biochim Biophys Acta 2011 [Epub ahead of print].

478. Anastasiadou E, Boccellato F, Vincenti S, Rosato P, Bozzoni I, Frati L, Faggioni C, Presdutti C and Trivedi P: Epstein-Barr virus encoded LMP-1 downregulates TCL1 oncogene through miR-29b. Oncogene 29: 1316-1328, 2010.

479. Cen O and Longnecker R: Rapamycin reverses splenomegaly and inhibits tumor development in a transgenic model of EpsteinBarr virus-related Burkitt's lymphoma. Mol Cancer Ther 10: 679-686, 2011

480. Horikawa T, Yoshikazi T, Kondo S, Furukawa M, Kaizaki Y and Pagano JS: Epstein-Barr virus latent membrane protein 1 induces Snail epithelial-mesenchymal transition in metastatic nasopharyngeal carcinoma. Br J Cancer 104: 1160-1167, 2011.

481. You S, Zhang F, Meng F, Li H, Liu Q, Liang Y, Dong Y, Yang W and Han A: EBV-encoded LMP1 increases nuclear $\beta$-catenin accumulation and its transcriptional activity in nasopharyngeal carcinoma. Tumour Biol 32: 623-630, 2011.

482. Kung CP, Meckes DG Jr and Raab-Traub N: Epstein-Barr virus LMP1 activates EGFR, STAT3, and ERK through effects on PKCdelta. J Virol 85: 4399-4408, 2011. 
483. Hannigan A, Qureshi AM, Nixon C, Tsimbouri PM, Jones S, Philbey AW and Wilson JB: Lymphocyte deficiency limits Epstein-Barr virus latent membrane protein 1 induced chronic inflammation and carcinogenic pathology in vivo. Mol Cancer 10: 11, 2011.

484. Ong KW, Teo M, Lee V, Ong D, Lee A, Tan CS, Vathsala A and Toh HC: Expression of EBV latent antigens, mammalian target of rapamycin, and tumor suppressor genes in EBV-positive smooth muscle tumors: clinical therapeutic implications. Clin Cancer Res 15: 5350-5358, 2009.

485. Sunde J, Chetty-John S, Shlobin OA and Boice CR: EpsteinBarr virus associated uterine leiomyosarcoma in an adult lung transplant patient. Obstet Gynecol 115: 434-436, 2010.

486. Morovic A, Delcore R and Damianov I: Inflammatory leiomyosarcoma of the retroperitoneum. Pathol Res Pract 199: 41-43, 2003.

487. Fillipakis H, Spandidos DA and Sourvinos G: Herpesviruses: hijacking the Ras signaling pathway. Biochim Biophys Acta 1803: 777-785, 2010a.

487. Mammas IN, Sourvinos G, Giannoudis A and Spandidos DA: Human papillomavirus (HPV) and host cell interactions. Pathol Oncol Res 14: 345-354, 2008b.

487. Jabbar SF, Abrams L, Glick A and Lambert PF: Persistence of high-grade cervical dysplasia and cervical cancer requires the continuous expression of the human papillomavirus type $16 \mathrm{E} 7$ oncogene. Cancer Res 69: 4407-4414, 2009c.

488. Al Moustafa AE, Chen D, Chabreau L and Akil N: Association between human papillomavirus and Epstein-Barr virus infections in human oral carcinogenesis. Med Hypotheses 73: 184-186, 2009.

489. Singhi AD, Stelow EB, Mills SE and Westra WH: Lymphoepithelial-like carcinoma of the oropharynx: a morphologic variant of HPV-related head and neck carcinoma. Am J Surg Pathol 34: 800-805, 2010.

490. Laborde RR, Novakova V, Olsen KD, Kasperbauer JL, Moore EJ and Smith DI: Expression profiles of viral responsive genes in oral and oropharyngeal cancers. Eur J Cancer 46: 1153-1158, 2010.

491. Maxwell JH, Kumar B, Feng FY, McHugh JB, Cordell KG Eisbruch A, Worden FP, Wolf GT, Prince ME, Moyer JS Teknos TN, Chepeha DB, Stoerker J, Walline H, Carey TE and Bradford CR: HPV-positive/p16-positive/EBV-negative nasopharyngeal carcinoma in white North Americans. Head Neck 32: $562-567,2010$

492. Ball SL, Winder DM, Vaughan K, Hanna N, Levy J, Sterling JC, Stanley MA and Goon PK: Analyses of human papillomavirus genotypes and viral loads in anogenital warts. J Med Virol 83: 1345-1350, 2011.

493. Frisch M, Glimelius B, van den Brule AJ, Wohlfahrt J, Meijer CJ, Walboomers JM, Adami HO and Melbye M: Benign anal lesions, inflammatory bowel disease and risk for high-risk human papillomavirus-positive and -negative anal carcinoma. Br J Cancer 78 . 1534-1538, 1998.

494. Singh M, Krajewski M, Mikolajka A and Holak TA: Molecular determinants for the complex formation between the retinoblastoma protein and LXCXE sequences. J Biol Chem 280: 37868-37876, 2005.

495. Liu X, Clements A, Zhao K and Marmorstein R: Structure of the human papillomavirus E7 oncoprotein and its mechanism for inactivation of the retinoblastoma tumor suppressor. J Biol Chem 281: 578-586, 2006

496. Chemes LB, Sánchez IE, Smal C and De Prat-Gay G: Targeting mechanism of the retinoblastoma tumor suppressor by a prototypical viral oncoprotein. Structural modularity, intrinsic disorder and phosphorylation of human papillomavirus E7. FEBS J 277: 973-988, 2010

497. Xu M, Katzenellenbogen RA, Grandori C and Galloway DA: NFX1 plays a role in human papillomavirus types 16 E6 activation of NFkappaB activity. J Virol 84: 11461-11469, 2010.

498. Korzeniewski N, Treat B and Duensing S: The HPV-16 E7 oncoprotein induces centriole multiplication through deregulation of Polo-like kinase 4 expression. Mol Cancer 10: 61, 2011

499. Chung $\mathrm{CH}$ and Gillison ML: Human papillomavirus in head and neck cancer: its role in pathogenesis and clinical implications. Clin Cancer Res 15: 6758-6762, 2009.

500. Sun SC: CYLD: a tumor suppressor deubiquitinase regulating NF-kappaB activation and diverse biological processes. Cell Death Differ 17: 25-34, 2010a.

500. Szalmás A, Bánáti F, Koroknai A, László B, Fehér E, Salamon D, Gergely L, Minárovits J and Kónya J: Lineage-specific silencing of human IL-10 gene expression by promoter methylation in cervical cancer cells. Eur J Cancer 44: 1030-1038, 2008b.
501. Pereira PM, Marques JP, Soares AR, Carreto L and Santos MA: MicroRNA expression variability in human cervical tissues. PLoS One 5: e11780, 2010.

502. Hu X, Schwarz JK, Lewis JS Jr, Huettner PC, Rader JS, Deasy JO, Grigsby PW and Wang X: A microRNA expression signature for cervical cancer prognosis. Cancer Res 70: 1441-1448, 2010.

503. Greco D, Kivi N, Qian K, Leivonen SK, Auvinen P and Auvinen E: Human papillomavirus 16 E5 modulates the expression of host microRNAs. PLoS One 6: e21646, 2011.

504. Trottier H, Ferreira S, Thomann P, Costa MC, Sobrinho JS, Prado JC, Rohan TE, Villa LL and Franco EL: Human papillomavirus infection and reinfection in adult women: the role of sexual activity and natural immunity. Cancer Res 70: 8569-8577, 2010

505. Shin MK, Balsitis S, Brake T and Lambert PF: Human papillomavirus E7 oncoprotein overrides the tumor suppressor activity of $\mathrm{p} 21^{\mathrm{CIP} 1}$ in cervical carcinogenesis. Cancer Res 69: 5656-5663, 2009.

506. Lepique AP, Daghastanli KR, Cuccovia IM and Villa LL: HPV16 tumor associated macrophages suppress antitumor T cell responses. Clin Cancer Res 15: 4391-4400, 2009.

507. Smith KA, Meisenburg BL, Tam VL, Pagarigan RR, Wong R, Joea DK, Lantzy L, Carrillo MA, Gross TM, Malyankar UM, Chiang CS, Da Silva DM, Kundig TM, Kast WM, Qiu Z and Bot A: Lymph node-targeted immunotherapy mediates potent immunity resulting in regression of isolated or metastatic human papillomavirus-transformed tumors. Clin Cancer Res 15: 6167-6176, 2009

508. Wei L, Gravitt PE, Song H, Maldonado AM and Ozbun MA: Nitric oxide induces early viral transcription coincident with increased DNA damage and mutation rates in human papillomavirus-infected cells. Cancer Res 69: 4878-4884, 2009a.

508. Zaravinos A, Kanellou P and Spandidos DA: Viral DNA detection and RAS mutations in actinic keratosis and nonmelanoma skin cancers. Br J Dermatol 162: 325-331, $2010 \mathrm{~b}$.

509. Chen CJ and Yang HI: Natural history of chronic hepatitis B revealed. J Gastroenterol Hepatol 26: 628-638, 2011.

510. Zhang D, Ma S, Zhang X, Zhao H, Ding H and Zeng C: Prevalent HBV point mutations found using an alternative search. BMC Infect Dis 10: 271, 2010.

511. Zhao C, Zhang W, Tian X, Fang C, Li H, Yuan Z, Yang P and Wen Y: Proteomic analysis of cell lines expressing small hepatitis B surface antigen revealed decreased glucose-regulated protein $78 \mathrm{kDa}$ expression in association with higher susceptibility to apoptosis. J Med Virol 82: 14-22, 2010.

512. Kew MC: Hepatitis B virus x protein in the pathogenesis of hepatitis B virus-induced hepatocellular carcinoma.J Gastroenterol Hepatol 26 (Suppl 1): 144-152, 2011.

513. Kong G,Zhang J,Zhang S, Shan C, Ye Land Zhang X: Upregulated microRNA-22a by hepatitis B virus X protein enhances hepatoma cell migration by targeting PREN in cell culture model. PLoS One 6: e19518, 2011

514. Raleigh DR, Marchiando AM, Zhang Y, Shen Y, Sasaki H, Wang Y, Long $M$ and Turner JR: Tight junction-associated MARVEL proteins marveld3, tricellulin, and occludin have distinct but overlapping functions. Mol Biol Cell 21: 1200-1213, 2010.

515. Gong Q, Cheng M, Chen H, Liu X, Si Y, Yang Y, Yuan Y, Jin C, Yang W, He F and Wang J: Phospholipid scramblase 1 mediates hepatitis C virus entry into host cells. FEBS Lett 585: 2647-2652, 2011.

516. Lee JW, Liao PC, Young KC, Chang CL, Chen SS, Chang TT, Lai MD and Wang SW: Identification of hnRNPH1, NF45, and C14orf166 as novel host interacting partners of the mature hepatitis C virus core protein. J Proteome Res 10: 4522-4534, 2011.

517. Jahan DS, Khaliq S, Ijaz B, Ahmad W and Hasan S: Role of $\mathrm{HCV}$ core gene of genotype $1 \mathrm{a}$ and $3 \mathrm{a}$ and host gene Cox-2 in HCV-induced pathogenesis. Virol J 8: 155, 2011.

518. Diesen DL and Kjuo PC: Nitric oxide and redox regulation in the liver: part II. Redox biology in pathologic hepatocytes and implications for intervention. J Surg Res 167: 96-112, 2011.

519. Khaliq S, Khaliq SA, Zahur M, Ijaz B, Jahan S, Ansar M, Riazuddin S and Hassan S: RNAi as a new therapeutic strategy against HCV. Biotechnol Adv 28: 27-34, 2010a.

519. Khaliq S, Jahan S, Ijaz B, Ahmad W, Asad S and Hassan S Inhibition of hepatitis $\mathrm{C}$ virus genotype 3 a by siRNAs targeting envelope genes. Arch Virol 156: 433-442, 2011b.

519. Khaliq S, Jahan S and Hassan S: Hepatitis C virus p7: molecular function and importance in hepatitis $\mathrm{C}$ virus life cycle and potential antiviral target. Liver Int 31: 606-617, 2011c. 
519. Khaliq S, Jahan S, Pervaiz A, Ali Ashfaq U and Hassan S: Downregulation of IRES containing 5'UTR of HCV genotype 3a using siRNAs. Virol J 8: 221, 2011d.

520. Libra M, Gloghini A, Malaponte G, Gangemi P, De Re V, Cacopardo B, Spandidos DA, Nicoletti F, Stivala F, Zignego AL and Carbone A: Association of $\mathrm{t}(14 ; 18)$ translocation with HCV infection in gastrointestinal MALT lymphomas. J Hepatol 49: 170-174, 2008.

521. Younossi ZM, Limongi D, Stepanova M, Pierobon M, Afendy A, Mehta R, Baranova A, Liotta L and Petricoin E: Protein pathway activation associated with sustained virologic responses in patients with chronic hepatitis $C$ treated with pegylated interferon (PEG-IFN) and ribavirin (RBV). J Proteome Res 102: 774-779, 2011.

522. Burdette D, Olivarez M and Waris G: Activation of transcription factor Nrf2 by hepatitis C virus induces the cell-survival pathway. J Gen Virol 91: 681-690, 2009.

523. Battaglia S, Benzoubir N, Nobilet S, Charneau P, Samuel D, Zignego AL, Atfi A, Bréchot C and Bourgeade MF: Liver cancerderived hepatitis $\mathrm{C}$ virus core proteins shift TGF-beta responses from tumor suppression to epithelial-to-mesenchymal transition. PLoS One 4: e4355, 2009.

524. Luedde TY, Beraza N, Kotsikoris V, van Loo G, Nenci A, De Vos R, Roskams T, Trautwein C and Pasparakis M: Deletion of NEMO/ IKKgamma in liver parenchymal cells causes steatohepatitis and hepatocellular carcinoma. Cancer Cell 11: 119-132, 2007.

525. Arrieta O, Rodriguez-Diaz JL, Rosas-Camargo V, MoralesEspinosa D, Ponce de Leon S, Kershenobich D and LeonRodriguez E: Colchicine delays the development of hepatocellular carcinoma in patients with hepatitis virus-related liver cirrhosis. Cancer 107: 1852-1858, 2006.

526. Hann HW: Telbivudine: an effective anti-HBV drug for chronic hepatitis B patients with early on-treatment responses. Expert Opin Pharmacother 11: 2243-2249, 2010.

527. Boldogkoi Z, Bratincsak A and Fodor I: Evaluation of pseudorabies virus as a gene transfer vector and an oncolytic agent for human tumor cells. Anticancer Res 22: 2153-2159, 2002.

528. Ye X, Lu Q, Zhao Y, Ren Z, Ren XW, Qiun OH, Tong Y, Liang M, Hu F and Chen HZ: Conditionally replicative adenovirus vector carrying TRAIL gene for enhanced oncolysis of human hepatocellular carcinoma. Int J Mol Med 16: 1179-1181, 2005.

529. Iankov ID, Blechacz B, Liu C, Schmeckpeper JD, Tarara JE, Federspiel MJ, Caplice N and Russell SJ: Infected cell carriers: a new strategy for systemic delivery of oncolytic measles virus in cancer virotherapy. Mol Ther 15: 114-122, $2007 \mathrm{a}$.

529. Jing Y, Tong C, Zhang J, Nakamura T, Iankov I, Russell SJ and Merchan JR: Tumor and vascular targeting of a novel oncolytic measles virus retargeted against the urokinase receptor. Cancer Res 69: 1459-1468, 2009b

530. Xue XB, Xiao CW, Zhang H, Lu AG, Gaio W, Zhou ZQ, Guo XL, Zhong MA, Yang Y and Wang CJ: Oncolytic adenovirus SG600IL24 selectively kills hepatocellular carcinoma cell lines. World J Gastroenterol 16: 4677-4684, 2010.

531. Chen W, Wu Y, Liu W, Wang G, Wang X, Yang Y, Chen W, Tai Y, Lu M, Qian Q, Zhang Q and Chen G: Enhanced antitumor efficacy of a novel fiber chimeric oncolytic adenovirus expressing p53 on hepatocellular carcinoma. Cancer Lett 307: 93-103, 2011.

532. Bose A, Taylor JL, Alber S, Watkins SC, Garcia JA, Rini BI, Ko JS, Cohen PA, Finke JH and Storkus WJ: Sunitinib facilitates the activation and recruitment of therapeutic tumor immunity in concert with specific vaccination. Int J Cancer 2010 [Epub ahead of print].

533. Blyth K, Vaillant F, Jenkins A, McDonald L, Pringle MA, Huseer C, Stein T, Neil J and Cameron ER: Runx2 in normal tissues and cancer cells: a developing story. Blood Cells Mol Dis 45: 117-123, 2010

534. Lin WC, Tsai HF, Kuo SH, Wu MS, Lin CW, Hsu PI, Cheng AL and Hsu PN: Translocation of Helicobacter pylori CagA into human B lymphocytes, the origin of mucosa-associated lymphoid tissue lymphoma. Cancer Res 70: 5740-5748, 2010.

535. Saito Y, Murata-Kamiya N, Hirayama T, Ohba $\mathrm{Y}$ and Hatakeyama M: Conversion of Helicobacter pylori CagA from senescence inducer to oncogenic driver through polarity-dependent regulation of p21. J Exp Med 207: 2157-2174, 2010.

536. Kuo SH, Cheng AL, Lin CW, Hsu CH, Wu MS, Yeh $\mathrm{KH}$, Tzeng YS and Chen LT: $t(11 ; 18)(\mathrm{q} 21 ; \mathrm{q} 21)$ translocation as predictive marker for non-responsiveness to salvage thalidomide therapy in patients with marginal zone B-cell lymphoma with gastric involvement. Cancer Chemother Pharmacol 2011 [Epub ahead of print].
537. Munari F, Lonardi S, Cassatella MA, Doglioni C, Cangi MG, Amedei A, Facchetti F, Eishi Y, Rugge M, Fassan M, De Bernard M, D'Ellios MM and Vermi W: Tumor-associated macrophages as major source of APRIL in gastric MALT lymphoma. Blood 117: 6612-6616, 2011.

538. Nakamura S, Sugiyama T, Matsumoto T, Iijima K, Ono S, Tajika M, Tari A, Kitadai Y, Matsumpto H, Nagaya T, Kamoshida T, Watanabe N, Chiba T, Origasa H, Asaka M and Japan GAST Study Group: Long-term clinical outcome of gastric MALT lymphoma after eradication of Helicobacter pylori: a multicentre cohort follow-up study of 420 patients in Japan. Gut 2011 [Epub ahead of print].

539. Romiti A, Zullo A, Borrini F, Sarcina I, Hassan C, Winn S, Tomao S, Vecchione A, Morini S and Mingazzini P: Relationship between beta-catenin expression and epithelial cell proliferation in gastric mucosa with intestinal metaplasia. World J Gastroenterol 11: 4400-4408, 2005.

540. Cao X, Tsukamoto T, Nozaki K, Mizoshuita T, Odasawara N, Tanaka H, Takenaka H, Takenaka Y, Kaminishi $\mathbf{M}$ and Tatematsu M: Beta-catenin gene alteration in glandular stomach adenocarcinomas in $\mathrm{N}$-methyl-N-nitrosourea-treated and Helicobacter pylori-infected Mongolian gerbils. Cancer Sci 95: 487-490, 2004.

541. Niwa T, Tsukamoto T, Toyoda T, Mori A, Tanaka H, Maekita T, Ichinose M, Tatematsu $\mathrm{M}$ and Ushijima T: Inflammatory process triggered by Helicobacter pylori infection cause aberrant DNA methylation in gastric epithelial cells. Cancer Res 70: 1430-1440, 2009.

542. Yan J, Zhang M, Zhang J, Chen X and Zhang X: Heliocobacter pylori infection promotes methylation of WWOX gene in human gastric cancer. Biochem Biophys Res Commun 408: 99-102, 2011.

543. Gao L, Michel A, Weck MN, Arndt V, Pawlita M and Brenner H: Helicobacter pylori infection and gastric cancer risk: evaluation of $\mathrm{H}$. pylori proteins determined by novel multiplex serology. Cancer Res 69: 6164-6170, 2009.

544. Baumgaertner I, Copie-Bergman C, Levy M, Haioun C, Charachon A, Bala M, Sobhani I and Delchier JC: Complete remission of gastric Burkitt's lymphoma after eradication of Helicobacter pylori. World J Gastroenterol 15: 5748-5750, 2009.

545. Buzás GM: Helicobacter pylori - 2010. Orv Hetil 151: 2003-2010, 2010.

546. Oliveira AL, Levada PM,Zanotti-Magalhaes EM, Magalhães LA and Ribeiro-Paes JT: Differences in the number of hemocytes in the snail host Biomphalaria tenagophila, resistant and susceptible to Schistosoma mansoni infection. Genet Mol Res 9: 2436-2445, 2010.

547. Zhang SM and Coultas KA: Identification and characterization of five transcription factors that are associated with evolutionarily conserved immune signaling pathways in the schistosome-transmitting Biomphalaria glabrata. Mol Immunol 48: 1868-1881, 2011.

548. Littlewood DT, Lockyer AE, Webster BL, Johnston DA and Le TH: The complete mitochondrial genomes of Schistosoma haematobium and Schistosoma spindale and the evolutionary history of mitochondrial genome changes among parasitic flatworms. Mol Phylogenet Evol 39: 452-467, 2006.

549. Han ZG, Brindley PJ, Wang SY and Chen Z: Schistosoma genomics: new perspectives on schistosome biology and hostparasite interaction. Annu Rev Genomics Hum Genet 10: 211-240, 2009.

550. Moné Y, Gourbal B, Duval D, Du Pasquier L, Kieffer-Jaquinod S and Mitta G: A large repertoire of parasite epitopes matched by a large repertoire of host immune receptors in an invertebrate host/parasite model. PLoS Negl Trop Dis 4: pii: e813, 2010.

551. Chaudhary KS, Lu QL, Abel PD, Khandan-Nia N, Shoma AM, El Baz M, Stamp GW and Lalani EN: Expression of bcl-2 and p53 oncoproteins in schistosomiasis-associated transitional and squamous cell carcinoma of urinary bladder. Br J Urol 79: 78-84, 1997.

552. Zalata KR, Nasif WA, Ming SC, Lofty M, Nada NA, El-Hak NG and Leech SH: p53, Bcl-2 and c-Myc expression in colorectal carcinoma associated with schistosomiasis in Egypt. Cell Oncol 27: 245-253, 2005.

553. Ramchurren N, Cooper JK and Summerhayes IC: Molecular events underlying schistosoma-related bladder cancer. Br J Cancer 62: 237-244, 1995.

554. El-Chennawl FA, AufFA, Metwally SS, Mosaad YM, Shasban AA, El-Baz MA, Tawhid ZE and Lofty ZF: Vascular endothelial growth factor, p53, and the H-ras oncogene in Egyptian patients with bladder cancer. World J Gastrointest Oncol 1: 62-68, 2009. 
555. Nakopoulou L, Vourlakou C,Zervas A, Tzonou A, Gakiopoulou $\mathrm{H}$ and Dimopoulos MA: The prevalence of bcl-2, p53, and Ki-67 immunoreactivity in transitional cell bladder carcinoma and their clinicopathologic correlates. Hum Pathol 29: 146-154, 1998.

556. Wolf DA, Wang S, Panzica MA, Bassikly NH and Thompson NK: Expression of a highly conserved oncofetal gene, TA1/E16, in human colon carcinoma and other primary cancers: homology to Schistosoma mansoni amino acid permease and Caenorhabditis elegans gene products. Cancer Res 56: 5012-5022, 1996.

557. Dissous C, Grevelding CG and Long T: Schistosoma mansoni polo-like kinases and their function in control of mitosis and parasite reproduction. An Acad Bras Cienc 83: 627-635, 2011.

558. Chaganti RS, Balazs I, Jhanwar SC, Murty VV, Kouru PR, Grzeschik KH and Staynezer E: The cellular homologue of the transforming gene SKV avian retrovirus maps to human chromosome region 1q22-q4. Cytogenet Cell Genet 43: 181-186, 1986.

559. Akiyoshin S, Inoue $H$, Hanai J, Kusanagi K, Nemoto $N$, Miyazono K and Kawataba M: c-SKI acts as a transcriptional co-repressor in transforming growth factor-beta signaling through interaction with smads. J Biol Chem 274: 35269-35277, 1998.

560. Laha T, Pinlaor P, Mulvenna J, Sripa B, Sripa M, Smout MJ, Gasser RB, Brindley PJ and Loukas A: Gene discovery for the carcinogenic human liver fluke, Opisthorchis viverrini. BMC Genomics 8: 189, 2007.

561. Boonmars T, Wu Z, Boojaruspinyo S, Pinlaor S, Nagano I, Takahashi Y, Kaewsamut B and Yongvanit P: Alteration of gene expression of RB pathway in Opisthorchis viverrini infectioninduced cholangiocarcinoma. Parasitol Res 105: 1273-1281, 2009.

562. Boonmars T, Wu Z, Boonjaruspinyo S, Puapairoj A, Kaewsamut B Nagano I, Pinlaor S, Yongvanit P, Wonkchalee O, Juassook A, Sudsarn P and Srisawangwong T: Involvement of c-Ski oncoprotein in carcinogenesis of cholangiocarcinoma induced by Opisthorchis viverrini and N-nitrosodimethylamine. Pathol Oncol Res 17: 219-227, 2011.

563. Lamouille $\mathrm{S}$ and Derynck RL: Oncogene and tumour suppressor: the two faces of SnoN. EMBO J 28: 3459-3460, 2009.

564. Ferrand N, Atfi A and Prunier C: The oncoprotein c-ski functions as a direct antagonist of the transforming growth factor-(beta) type I receptor. Cancer Res 70: 8457-8466, 2010.

565. Fried B, Reddy A and Mayer D: Helminths in human carcinogenesis. Cancer Lett 305: 239-249, 2011.

566. Jahchan NS and Luo K: SnoN in mammalian development, function and diseases. Curr Opin Pharmacol 10: 670-676, 2010.

567. Sinkovics JG and Horvath JC: Viral contaminations of the poliomyelitis vaccines. Acta Microbiol Immunol Hung 47: 471-475, 2000.

568. Fioriti D, Pietropaolo V, Dal Forno S, Laurenti C, Chiarini F and Degener AM: Urothelial bladder carcinoma and viral infections: different association with human polyomaviruses. Int J Immunopathol Pharmacol 16: 283-288, 2003.

569. Mertz KD, Junt T, Schmid M, Pfaltz M and Kempf W: Inflammatory monocytes are a reservoir for Merkel cell polyomavirus. J Invest Dermatol 130: 1146-1151, 2010

570. Gjoerup O and Chang Y: Update on human polyomaviruses and cancer. Adv Cancer Res 106: 1-51, 2010.

571. Welsh JS: Contagious cancer. The Oncologist 16: 1-4, 2011.

572. Bauman Y, Nachmani D, Vitenshtein A, Tsukerman P, Drayman N, Stern-Ginossar N, Lankry D, Gruda R and Mandelboim O: An identical miRNA of the human JC and BK polyoma viruses targets the stress-induced ligand ULBP3 to escape immune elimination. Cell Host Microbe 9: 93-102, 2011.

573. Cotroneo MS, Haag JD, Stapel NR, Waller JL, Woditschka S and Gould MN: Freund's vaccine adjuvant promotes Her2/neu breas cancer. BMC Cancer 9: 19, 2009.

574. Du Q, Zhang X, Cardinal J, Cao Z, Guo Z, Shao Q and Geller DA: Wnt/beta-catenin signaling regulates cytokine-induced human inducible nitric oxide synthase expression by inhibiting nuclear factor kappaB activation in cancer cells. Cancer Res 69: 3764-3771, 2009.

575. Fu L, Kim YA, Wang X, Wu X, Yue P, Lonial S, Khuri FR and Sun SY: Perifosine inhibits mammalian target of rapamycin signaling through facilitating degradation of major components in the mTOR axis and induce autophagy. Cancer Res 69: 8967-8976, 2009.

576. Kiyono K, Suzuki H, Matsuyama H, Morishita Y, Komuro A, Kano MR, Sugimoto K and Miyazono K: Autophagy is activated by TGF- $\beta$ and potentiates TGF- $\beta$-mediated growth inhibition in human hepatocellular carcinoma cells. Cancer Res 69: 8844-8852, 2009.
577. Maiuri MC, Criollo A and Kroemer G: Crosstalk between apoptosis and autophagy within the Beclin 1 interactome. EMBO J 29: 515-516, 2010 .

578. Amaravadi RK, Lippincott-Schwartz J, Yin XM, Weiss WA, Takebe N, Timmer W, Di Paola RS, Lotze MT and White E: Principles and current strategies for targeting autophagy for cancer treatment. Clin Cancer Res 17: 654-666, 2011.

579. Delmas D, Solary E and Latruffe N: Resveratrol, a phytochemical inducer of multiple cell pathways: apoptosis, autophagy, and mitotic catastrophe. Curr Med Chem 18: 1100-1121, 2011.

580. Puissant A, Robert G, Fenouille N, Luciano F, Cassuto JP, Raynaud $S$ and Auberger P: Resveratrol promotes autophagic cell death in chronic myelogenous leukemia cells via JNK-mediated p62/SQSTM1 expression and AMPK activation. Cancer Res 70: 1042-1052, 2010.

581. Subramaniam D, Ramalingam S, Linehan DC, Dieckgraefe BK, Postier RG, Houchen CW, Jensen RA and Anant S: RNA binding protein CUGBP2/CELF2 mediates curcumin-induced mitotic catastrophe of pancreatic cancer cells. PLoS One 6: e16958, 2011.

582. Pimkina J, Humbey O, Zilfou JT, Jarnik M and Murphy ME: ARF induces autophagy by virtue of interaction with Bcl-xl. J Biol Chem 284: 2803-2810, 2009.

583. White E and Di Paola RS: The double-edged sword of autophagy modulation in cancer. Clin Cancer Res 15: 5308-5316, 2009a.

583. White E, Karp C, Strohecker AM, Guo Y and Mathew R: Role of autophagy in suppression of inflammation and cancer. Curr Opin Cell Biol 22: 212-217, 2010b.

584. Salamon D, Banati F, Koroknai A, Ravasz M, Szenthe K, Bathori Z, Bakos A, Niller HH, Wolf $\mathrm{H}$ and Minarovits J: Binding of CCCTC-binding factor in vivo to the region located between Rep $^{*}$ and the $\mathrm{C}$ promoter of Epstein-Barr virus is unaffected by $\mathrm{CpG}$ methylation and does not correlate with $\mathrm{Cp}$ activity. J Gen Virol 90: 1183-1189, 2009.

585. Fejer G, Koroknai A, Banati F, Györy I, Salamon D, Wolf H, Nillier HH and Minarovits J: Latency type-specific distribution of epigenetic marks at the alternative promoters $\mathrm{Cp}$ and $\mathrm{Qp}$ of Epstein-Barr virus. J Gen Virol 89: 1364-1370, 2008.

586. Perl A, Fernandez D, Telarico T and Phillips PE: Endogenous retroviral pathogenesis in lupus. Curr Opin Rheumatol 22: 483-492, 2010.

587. Sinkovics JG: Working hypothesis: viral etiology of autoimmune diseases. N Engl J Med 260: 903-904, 1969a.

587. Sinkovics JG, Gyorkey $F$ and Thoma GW: A rapidly fatal case of systemic lupus erythematosus: structures resembling viral nucleoprotein strands in the kidney and activities of lymphocytes in culture. Tex Rep Biol Med 27: 887-908, $1969 \mathrm{~b}$.

588. Gyorkey F, Sinkovics JG, Min KW and Gyorkey P: A morphological study on the occurrence and distribution of structures resembling viral nucleocapsids in collagen diseases. Am J Med 53: $148-158,1972$

589. Ekström Smedby K, Vajdic CM, Falster M, Engels EA, MartinezMaza O, Turner J, Hjalgrim H, Vineis P, Seniori Costantini A, Bracci PM, Holly EA, Willett E, Spinelli JJ, La Vecchia C, Zheng T, Becker N, De Sanjosé S, Chiu BC, Dal Maso L, Cocco P, Maynadié M, Foretova L, Staines A, Brennan P, Davis S Severson R, Cerhan JR, Breen EC, Birmann B, Grulich AE and Cozen W: Autoimmune disorders and risk of non-Hodgkin lymphoma subtypes: a pooled analysis within the InterLymph Consortium. Blood 111: 4029-4038, 2008.

590. Bernatsky S, Ramsey-Goldman R, Folkes WD, Gordon C and Clarke AE: Breast, ovarian, and endometrial malignancies in systemic lupus erythematosus: a meta-analysis. Br J Cancer 104: 1478-1481, 2011.

591. Bernatsky S, Ramsey-Goldman R, Gordon C and Clarke AE: Prostate cancer in systemic lupus erythematosus. Int J Cancer doi: 10.1002/ijc.25956, 2011.

592. Hemminki K, Liu X, Ji J, Sunquist J and Sundquist K: Autoimmune disease and subsequent digestive tract cancer by histology. Ann Oncol 2011 [Epub ahead of print].

593. Leng RX, Pan HF, Qin WZ, Chen GM and Ye DQ: Role of microRNA-155 in autoimmunity. Cytokine Growth Factor Rev 22: $141-147,2011$

594. Ankathatti Munegowda M, Deng Y, Mulligan SJ and Xiang J: Th17 and Th17-stimulated CD8(+) T cells play a distinct role in Th17 induced preventive and therapeutic antitumor immunity. Cancer Immunol Immunother 60: 1473-1484, 2011.

595. Kryczek I, Wu K, Zhao E, Wei S, Vatan L, Szeliga W, Huang E, Greenson J, Chang A, Rolinski J, Radwan P, Fang J, Wang G and Zou W: IL-17 regulatory T cells in the microenvironment of chronic inflammation and cancer. J Immunol 186: 4388-4396, 2011. 
596. Federico A, Morgillo F, Tuccillo C, Ciardiello F and Loguercio C: Chronic inflammation and oxidative stress in human carcinogenesis. Int J Cancer 121: 2381-2386, 2007.

597. Nishikawa M: Reactive oxygen species in tumor metastasis. Cancer Lett 166: 53-59, 2008.

598. Franco R, Schoneveld O, Georgakilas AG and Panjayiotidis MI: Oxidative stress, DNA methylation and carcinogenesis. Cancer Lett 266: 6-11, 2008.

599. Trompet S, De Craen AJ, Mooijaart S, Stott DJ, Ford I, Sattar N, Jukema W and Westendorp RG: High innate production capacity of proinflammatory cytokines increases risk for death from cancer: results of the PROSPER study. Clin Cancer Res 15: 7744-7748, 2009.

600.Cooke MS, Evans MD, Dizdaroglu M and Lunec J: Oxidative DNA damage: mechanisms, mutation, and disease. FASEB J 17: 1195-1214, 2003.

601. Sinkovics JG: Immunology in tumors in experimental animals In: The Immunology of Malignant Disease. Harris JE and Sinkovics JG (eds). 2nd edition. CV Mosby, St. Louis, MO, pp93-282, 1976a.

601. Gőcze K, Gombos K, Pajkos G, Magda I, Ember A, Juhász K, Patczai B and Ember L: Impact of microRNAs on molecular epidemiology. Orv Hetil 152: 633-641, 2011b.

602. Spandidos DA: A unified theory for the development of cancer. Biosci Rep 6: 691-708, 1986.

603. Lee DH, Severin K, Yokobatashi Y and Ghadiri MR: Emergence of symbiosis in peptide self-replication through a hypercycle network. Nature 390: 591-594, 1997.

604. Domingo-Espin J, Vazquez E, Ganz J, Conchillo O, GarcíaFruitós E, Cedano J, Unzueta U, Petegnief V, GonzalezMontalbán N, Planas AM, Daura X, Peluffo H, Ferrer-Miralles N and Villaverde A: Nanoparticulate architecture of protein-based artificial viruses is supported by protein-DNA interactions. Nanomedicine (Lond) 6: 1047-1061, 2011.

605. Kristensen DM, Mushegian AR, Dolja VV and Koonin EV: New dimensions of the virus world discovered through metagenomics. Trends Microbiol 18: 11-19, 2010.

606. Wu D, Wu M, Halpern A, Rusch DB, Yooseph S, Frazier M, Venter JC and Eisen JA: Stalking the fourth domain in metagenomic data: searching for, discovering, and interpreting novel, deep branches in marker gene phylogenetic trees. PLoS One 6 : e18011, 2011.

607. Sinkovics JG: The place of viruses in the 'Tree of Life'. Acta Microbiol Immunol Hung 48: 115-127, 2001.

608. Parris GE: The role of viruses in cell fusion and the importance to evolution, invasion and metastasis of cancer clones. Med Hypotheses 64: 1011-1014, 2005.

609. Gao P and Zheng J: Oncogenic virus-mediated cell fusion: new insight into initiation and progression of oncogenic virusesrelated cancers. Cancer Lett 303: 1-8, 2011.

610. Sinkovics JG, Drewinko B and Thornell E: Immunoresistant tetraploid lymphoma cells. Lancet i: 139-140, 1970a.

610. Sinkovics JG, Shirato E, Gyorkey F, Cabiness JR and Howe CD Relationship between lymphoid neoplasms and immunologic functions. In: Leukemia-Lymphoma. The 14th Annual Clinical Conference on Cancer, 1969. The University of Texas M.D. Anderson Hospital, Houston, TX. Year Book Medical Publisher, Chicago, IL, pp53-92, 1970b.

611. Sinkovics JG: The earliest concept of the 'hybridoma principle' recognized in 1967-1968. Front Radiat Ther Oncol 24: 18-31, 1990.

612. Sinkovics JG: A notable phenomenon recapitulated. A fusion product of a murine lymphoma cell and a leukemia virus-neutralizing antibody-producer host plasma cell formed spontaneously and secreting the specific antibody continuously. Acta Microbiol Immunol Hung 52: 1-40, 2005a.

612. Sinkovics JG: The first observation (in the late 1960s) of fused lymphoid cells continuously secreting a specific antibody. Bull Mol Med 26: 61-80, 2005b.

613. Sinkovics JG: Natural and artificial hybridoma formations by cell fusions. In: Cytolytic Immune Lymphocytes. Schenk Buchverlag, Passau and Budapest, pp92-114, 2008.

614. Wainwright M: The Sinkovics hybridoma - the discovery of the first 'natural hybridoma'. Prospect Biol Med 35: 372-379, 1992.
615. Koonin EV, Wolf YI, Nagasaki K and Dolja VV: The Big Bang of picorna-like virus evolution antedates the radiation of eukaryotic supergroups. Nat Rev Microbiol 6: 925-939, 2008.

616. Villarreal LP and Witzany G: Viruses are essential agents within the roots and stem of the tree of life. J Theor Biol 262: 698-710, 2010.

617. Fritz-Laylin JK and Cande Z: Ancestral centriol and flagella proteins identified by analysis of Naegleria differentiation. Cell Sci 123: 4024-4031, 2010.

618. Fritz-Laylin LK, Prochnik SE, Ginger ML, Dacks JB, Carpenter ML, Field MC, Kuo A, Paredez A, Chapman J, Pham J, Shu S, Neupane R, Cipriano M, Mancuso J, Tu H, Salamov A, Lindquist E, Shapiro H, Lucas S, Grigoriev IV, Cande WZ, Fulton C, Rokhsar DS and Dawson SC: The genome of Naegleria gruberi illuminates early eukaryotic versatility. Cell 140: 631-642, 2010

619. Pelle R, Graham SP, Njahira MN, Osaso J, Saya RM, Odongo DO, Toye PG, Spooner PR, Musoke AJ, Mwangi DM, Taracha EL, Morrison WI, Weir W, Silva JC and Bishop RP: Two Theileria parva CD8 T cell antigen genes are more variable in buffalo than cattle parasites, but differ in pattern of sequence diversity. PLoS One 6: e19015, 2011.

620. Chaussepied M, Janski N, Baumgartner M, Lizundia R, Jensen K, Weir W, Shiels BR, Weitzman JB, Glass EJ, Werling D and Langsley G: TGF- $\beta 2$ induction regulates invasiveness of Theileria-transformed leukocytes and disease susceptibility. PLoS Pathog 6: e1001197, 2010.

621. Havashida K, Hattori M, Nakao R, Tanaka Y, Kim JY, Inoue N, Nene V and Sugimoto C: A schizont-derived protein, TpSCOP, is involved in the activation of NF-kappaB in Theileria parvainfected lymphocytes. Mol Biochem Parasitol 174: 8-17, 2010.

622. Vlashi E, Lagadec C, Vergnes L, Matsutani T, Masui K, Poulou M, Popescu R, Della Donna L, Evers P, Dekmezian C, Reue K, Christofk H, Mischel PS and Pajonk F: Metabolic state of glioma stem cells and nontumorigenic cells. Proc Natl Acad Sci USA 108: 16062-16067, 2011.

623. Ivanov A, Cragg MS, Erenpreisa J, Emzinsh D, Lukman H and Illidge TM: Endopolyploid cells produced after severe genotoxic damage have the potential to repair DNA strand breaks. J Cell Sci 116: 4095-4106, 2003.

624. Lowden R, Flibotte S, Moerman DG and Ahmed S: DNA synthesis generates terminal duplications that seal end-to-end chromosome fusions. Science 332: 468-471, 2011.

625. Flügel RM: The precellular scenario of genovirions. Virus Genes 40: $151-154,2010$.

626. Woo HJ and Wallqvist A: Nonequilibrium phase transitions associated with DNA replication. Phys Rev Lett 106: 060601, 2011.

627. Arguello F: Atavistic metamorphosis. Amazon, pp1-126, 2011a

627. Duesberg P, Mandrioli D, McCormack A and Nicholson JM: Is carcinogenesis a form of speciation? Cell Cycle 10: 2100-2114, $2011 b$.

628. Sinkovics JG: Molecular biology of oncogenic inflammatory processes. II. The oncogenic DNA as a replica of the primordial DNA. In manuscript

629. Koonin EV, Senkevich TG and Dolja VV: The ancient virus world and evolution of cells. Biol Direct 1: 29, 2006.

630. Obermayer B, Krammer H, Braun D and Gerland U: Emergence of information transmission in a prebiotic RNA reactor. Phys Rev Lett 107: 018101, 2011

631. Cech TR: Evolution of biological catalysis: ribozyme to RNP enzyme. Cold Spring Harb Symp Quant Biol 74: 11-16, 2009.

632. Diener TO: The viroid: biological oddity or evolutionary fossil? Adv Virus Res 57: 137-164, 2001

633. Hegedus K, Palkovics L, Tóth EK, Dallmann G and Balázs E: The DNA from of a retroviroid-like element characterized in cultivated carnation species. J Gen Virol 82: 687-691, 2001.

634. Taylor $J$ and Pelchat M: Origin of hepatitis $\delta$ virus. Future Microbiol 5: 393-402, 2010.

635. Flores R, Grubb D, Elleuch A, Nohales MA, Delgado S and Gago S: Rolling-cycle replication of viroids, viroid-like satellite RNAs and hepatitis delta virus: variations on a theme. RNA Biol 8: 200-206, 2011. 